\title{
A multipoint conformal block chain in $d$ dimensions
}

\author{
Sarthak Parikh \\ Division of Physics, Mathematics and Astronomy, California Institute of Technology, \\ Pasadena, CA 91125, U.S.A. \\ E-mail: sparikh@caltech.edu
}

ABSTRACT: Conformal blocks play a central role in CFTs as the basic, theory-independent building blocks. However, only limited results are available concerning multipoint blocks associated with the global conformal group. In this paper, we systematically work out the $d$-dimensional $n$-point global conformal blocks (for arbitrary $d$ and $n$ ) for external and exchanged scalar operators in the so-called comb channel. We use kinematic aspects of holography and previously worked out higher-point AdS propagator identities to first obtain the geodesic diagram representation for the $(n+2)$-point block. Subsequently, upon taking a particular double-OPE limit, we obtain an explicit power series expansion for the $n$-point block expressed in terms of powers of conformal cross-ratios. Interestingly, the expansion coefficient is written entirely in terms of Pochhammer symbols and $(n-4)$ factors of the generalized hypergeometric function ${ }_{3} F_{2}$, for which we provide a holographic explanation. This generalizes the results previously obtained in the literature for $n=4,5$. We verify the results explicitly in embedding space using conformal Casimir equations.

KeYwords: Conformal Field Theory, AdS-CFT Correspondence, Conformal and W Symmetry

ARXiv EPrint: 1911.09190

Dedicated to the memory of Steven S. Gubser. 


\section{Contents}

1 Introduction 1

2 Low-point examples of holographic duals 5

2.1 Holographic dual of the six-point block 6

$\begin{array}{lll}2.2 & \text { OPE limit of the six-point block } & 10\end{array}$

2.2.1 Recovering the four-point block from the six-point block 11

3 Multipoint block in the comb channel $\quad \mathbf{1 4}$

3.1 Holographic dual of the $(n+2)$-point block and its double-OPE limit 14

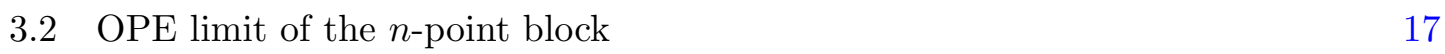

$\begin{array}{lll}3.3 \text { Conformal Casimir check } & 19\end{array}$

$\begin{array}{llr}4 & \text { Discussion } & 26\end{array}$

$\begin{array}{ll}\text { A Obtaining the six-point holographic dual } & 30\end{array}$

B Five- and seven-point examples $\quad 35$

$\begin{array}{lll}\text { B.1 Double-OPE limit and the five-point block } & 37\end{array}$

$\begin{array}{lll}\text { C Further technical details } & \mathbf{3 8}\end{array}$

$\begin{array}{lll}\text { C.1 Proof of (3.15) } & 38\end{array}$

$\begin{array}{lll}\text { C.2 Proof of (3.32) } & 39\end{array}$

$\begin{array}{lll}\text { C.3 Proof of (3.37) } & 40\end{array}$

\section{Introduction}

Conformal field theories (CFTs) are important for several reasons - they serve as salient guideposts in the space of quantum field theories, describe a variety of critical phenomena, and help elucidate aspects of quantum gravity via the AdS/CFT correspondence [1-3]. Conformal blocks play a central role in CFTs. They are the basic kinematic building blocks of local observables, encoding the contribution of primary operators (and all their descendants) to any given correlation function. Given a $d$-dimensional CFT (more precisely the dynamical data in the form of the spectrum of all primary operators and the operator product expansion (OPE) coefficients between them), the knowledge of $d$-dimensional conformal blocks permits the explicit writing of all possible correlators. Conversely, the conformal bootstrap program [4-6] (see also the recent review [7] and references therein) provides a non-perturbative approach to reconstructing the full $\mathrm{CFT}_{d}$ data by exploiting conformal symmetry as well as stringent consistency conditions such as the associativity of the OPE. Here as well, conformal blocks are an essential ingredient needed for setting up the bootstrap equations involving conformal correlators. 
It is therefore important to understand these basic building blocks in as much detail as possible. In this paper we will be focusing on multipoint $d$-dimensional global conformal blocks associated with the Euclidean conformal group $\mathrm{SO}(d+1,1)$. While these theory independent objects are in principle fixed entirely from conformal symmetry, working them out presents significant computational challenges, so explicit results are available in only simple cases. For instance, explicit expressions are known for four-point scalar conformal blocks in general spacetime dimensions [8-13]. A variety of techniques have been developed for computing four-point conformal blocks involving external and internal exchanged operators in arbitrary representations of the Lorentz group in closed-form, integral or efficient series expansions; a partial list includes various recursive methods [11, 13-21], shadow formalism [22], use of differential operators [23-35], Wilson line constructions [36-38], integrability methods [39-42] and holographic geodesic diagram techniques [43-52]. The situation is disproportionately dire for higher-point global conformal blocks. Recent work in the shadow formalism has led to explicit series expansions for $n$-point scalar conformal blocks in dimensions one and two in a specific channel called the comb channel, for arbitrary $n$ [53]. In higher dimensions, a series expansion was obtained for the scalar five-point block restricted to the exchange of scalar representations [53] (see also ref. [54]). Geodesic diagram representations have also been obtained for the same five-point block in general spacetime dimensions [55], as well as for the $d$-dimensional six-point scalar conformal block involving scalar exchanges in a different channel called the OPE channel [56].

While one can recursively reduce any higher-point conformal correlator into a combination of two- and three-point functions via repeated use of the OPE (or equivalently, reduce to a combination of four-point correlation functions), higher-point correlators and conformal blocks are important in their own right for a number of reasons. For one, knowledge of higher-point blocks allows immediately an efficient writing of conformal correlators and repackaging of higher-point AdS diagrams directly in position space. Moreover, higher-point diagrams involve exchange of multi-twist exchanges in their conformal block decomposition, which can provide a new window into understanding multi-twist exchanges appearing in the setting of light-cone bootstrap of four-point functions [57-66]. Additionally, knowledge of higher-point scalar conformal blocks opens up the possibility of setting up an equivalent but possibly more efficient alternative to the conventional bootstrap program. In the conventional approach, typically one must study crossing equations of four-point correlators of all operators in the spectrum including those in non-trivial representations of the Lorentz group. As a potential alternative, one can instead aim to solve crossing equations for scalar $n$-point functions, but for all $n$ [53,67]. Clearly, $n$-point blocks will play a crucial role here.

Motivated by these considerations, in this paper we will compute all higher-point $d$ dimensional global conformal blocks in a channel which ref. [53] referred to as the comb channel, for external and exchanged scalar operators (see figure 1). This will generalize the series expansion for the $d$-dimensional five-point block computed in ref. [53] to $n$-point blocks for any $n$. The main techniques we will be employing are the AdS propagator identities and geodesic diagram techniques of refs. [43, 48, 55, 56]. Our strategy will be to obtain the holographic geodesic diagram representation of an $(n+2)$-point block in the comb 


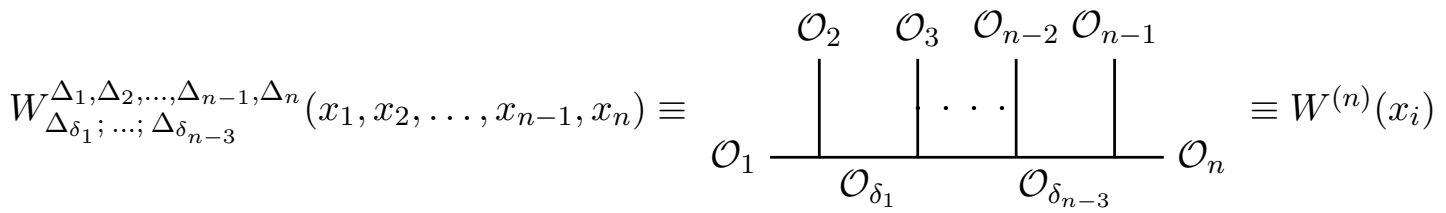

Figure 1. The comb channel $n$-point global conformal block for external scalar operators $\mathcal{O}_{1}\left(x_{1}\right), \ldots, \mathcal{O}_{n}\left(x_{n}\right)$ with conformal dimensions $\Delta_{1}, \ldots, \Delta_{n}$ and insertion coordinates $x_{1}, \ldots, x_{n}$ respectively, and exchanged scalar operators $\mathcal{O}_{\delta_{1}}, \ldots, \mathcal{O}_{\delta_{n-3}}$ with conformal dimensions $\Delta_{\delta_{1}}, \ldots, \Delta_{\delta_{n-3}}$, respectively. When there is no scope for confusion, we will often abbreviate it as $W^{(n)}\left(x_{i}\right)$ or simply $W^{(n)}$.

channel with the help of various recently derived AdS propagator identities [56]. Higherpoint geodesic diagrams, like the four-point case [43], are higher-point AdS diagrams where all bulk integrations are restricted to geodesic integrals, and they are related to higher-point conformal blocks $[55,56]$. For comb channel blocks, these geodesic diagram representations involve precisely two geodesic integrals. Taking a particular double-OPE limit gets rid of these geodesic integrals, producing an $n(n-3) / 2$-fold power series expansion of the $n$-point conformal block. We also verify our result via a proof by conformal Casimir equations.

This paper is organized as follows. In section 2 we illustrate the main computational strategy in the simplest non-trivial example. Particularly, in section 2.1 (along with appendix A) we derive the holographic geodesic diagram representation of the six-point comb channel block, and in section 2.2 we reproduce the well-known series expansion of the fourpoint block by taking a double-OPE limit. A second example is provided in appendix B, where we briefly discuss the holographic seven-point block and its double-OPE limit which recovers the series expansion of the five-point block. In section 3, with the help of these examples, we propose a holographic representation of the general $(n+2)$-point block, whose double-OPE limit leads to the power series expansion of the $n$-point block. Sections 3.2-3.3 are concerned with proving our claim via conformal Casimir equations. We conclude in section 4 with comments on extending the results to multipoint scalar blocks in arbitrary channels, brief remarks on the comparison with analogous results in the framework of $p$ adic AdS/CFT, and a proposal for an alternate, more rapidly convergent series expansion for $n$-point blocks in the comb channel. Further computational details can be found in appendix $\mathrm{C}$.

We end this section with a presentation of the main technical result of this paper, which is an explicit power series expansion for the comb channel $n$-point global conformal block in $d$ spacetime dimensions for external and exchanged scalar operators (see figure 1): ${ }^{1}$

$$
\begin{gathered}
W^{(n)}\left(x_{i}\right)=\frac{\prod_{t=0}^{n-3} \Gamma\left(1-\Delta_{\delta_{t} \delta_{t+1},(t+2)}\right)}{\Gamma\left(1-\Delta_{1 n, 2 \ldots(n-1)}\right) \prod_{i=1}^{n-3} \Gamma\left(1-\Delta_{\delta_{i}}\right)} W_{0}^{(n)}\left(x_{i}\right)\left(\prod_{i=1}^{n-3} u_{i}^{\frac{\Delta_{\delta_{i}}}{2}}\right) \\
\times \sum_{\substack{k_{1}, \ldots, k_{n-3}, j_{\langle 2 \mid 3\rangle}, j_{\langle 2 \mid 4\rangle}, \ldots, j_{\langle n-2 \mid n-1\rangle}=0}}^{\infty}\left[\left(\prod_{i=1}^{n-3} \frac{u_{i}^{k_{i}}}{k_{i} !}\right)\left(\prod_{2 \leq r<s \leq n-1} \frac{\left(-w_{r ; s}\right)^{j_{\langle r \mid s\rangle}}}{j_{\langle r \mid s\rangle} !}\right)\right.
\end{gathered}
$$

\footnotetext{
${ }^{1}$ For convenience, this result is also included with the submission in the supplementary material as a Mathematica notebook.
} 


$$
\begin{aligned}
& \times\left(\Delta_{1 n, 2 \ldots(n-1)}\right)_{-\sum_{2 \leq r<s \leq n-1} j_{\langle r \mid s\rangle}}\left(\prod_{t=0}^{n-3} \frac{\left(1-\Delta_{(t+1) \delta_{t-1}, \delta_{t}}\right)_{k_{t}}\left(1-\Delta_{(t+2) \delta_{t+1}, \delta_{t}}\right)_{k_{t}}}{\left(\Delta_{\delta_{t}}-d / 2+1\right)_{k_{t}}}\right. \\
& \times\left(\Delta_{(t+2) \delta_{t}, \delta_{t+1}}\right)_{k_{t, t+1}+\sum_{2 \leq r<t+2} j_{\langle r \mid t+2\rangle}}\left(\Delta_{(t+2) \delta_{t+1}, \delta_{t}}\right)_{k_{t+1, t}+\sum_{t+2<s \leq n-1} j_{\langle t+2 \mid s\rangle}} \\
& \left.\left.\times{ }_{3} F_{2}\left[\left\{-k_{t},-k_{t+1}, \Delta_{\delta_{t} \delta_{t+1}(t+2),}-\frac{d}{2}\right\} ;\left\{\Delta_{(t+2) \delta_{t+1}, \delta_{t}}-k_{t}, \Delta_{(t+2) \delta_{t}, \delta_{t+1}}-k_{t+1}\right\} ; 1\right]\right)\right],
\end{aligned}
$$

where $(a)_{b} \equiv \Gamma(a+b) / \Gamma(a)$ is the Pochhammer symbol and we are using the notation

$$
\Delta_{i_{1} \ldots i_{\ell}, i_{\ell+1} \ldots i_{k}} \equiv \frac{1}{2}\left(\Delta_{i_{1}}+\cdots+\Delta_{i_{\ell}}-\Delta_{i_{\ell+1}}-\cdots-\Delta_{i_{k}}\right)
$$

for conformal dimensions $\Delta_{i}$, whereas

$$
k_{i_{1} \ldots i_{\ell}, i_{\ell+1} \ldots i_{n}} \equiv k_{i_{1}}+\cdots+k_{i_{\ell}}-k_{i_{\ell+1}}-\cdots-k_{i_{n}}
$$

for the integral parameters $k_{i}$, as well as the additional definitions ${ }^{2}$

$$
k_{0} \equiv 0 \quad k_{n-2} \equiv 0 \quad \Delta_{\delta_{0}} \equiv \Delta_{1} \quad \Delta_{\delta_{n-2}} \equiv \Delta_{n},
$$

so that there are precisely $(n-3)$ independent $k_{i}$ parameters to be summed over in (1.1). The other set of integral parameters is denoted $j_{\langle r \mid s\rangle}$, for $2 \leq r<s \leq n-1$, where we use the notation $\langle\cdot \mid \cdot\rangle$ in the subscript to index the $\left(\begin{array}{c}n-2 \\ 2\end{array}\right)$ independent $j$ parameters. ${ }^{3}$ Combined, this leads to an $n(n-3) / 2$-fold sum.

The coordinate dependence of the conformal block (1.1) is factorized into a "leg factor", which depends solely on external dimensions and is given by

$$
W_{0}^{\Delta_{1}, \ldots, \Delta_{n}}\left(x_{1}, \ldots, x_{n}\right) \equiv\left(\frac{x_{2 n}^{2}}{x_{1 n}^{2} x_{12}^{2}}\right)^{\frac{\Delta_{1}}{2}}\left(\frac{x_{1(n-1)}^{2}}{x_{1 n}^{2} x_{(n-1) n}^{2}}\right)^{\frac{\Delta_{n}}{2}} \prod_{i=2}^{n-1}\left(\frac{x_{1 n}^{2}}{x_{1 i}^{2} x_{i n}^{2}}\right)^{\frac{\Delta_{i}}{2}} \equiv W_{0}^{(n)}\left(x_{i}\right),
$$

where $x_{i j}=x_{i}-x_{j}$, while the rest of the dependence is expressible as an $n(n-3) / 2$-fold power series expansion entirely in terms of a set of $n(n-3) / 2$ independent $^{4}$ cross-ratios $^{2}$ $0 \leq u_{i}, w_{r ; s} \leq 1$ defined as follows, ${ }^{5}$

$$
u_{i} \equiv \frac{x_{1(i+1)}^{2} x_{(i+2) n}^{2}}{x_{(i+1) n}^{2} x_{1(i+2)}^{2}} \quad 1 \leq i \leq n-3, \quad w_{r ; s} \equiv \frac{x_{1 n}^{2} x_{r s}^{2}}{x_{r n}^{2} x_{1 s}^{2}} \quad 2 \leq r<s \leq n-1 .
$$

\footnotetext{
${ }^{2}$ The apparent dependence of the conformal block (1.1) on the undefined dimension $\Delta_{\delta_{-1}}$ is spurious, since $\Delta_{\delta_{-1}}$ always appears inside Pochhammer symbols of the form $(a)_{0}$ for some non-zero $a$, which evaluate identically to unity.

${ }^{3}$ This notation should not be confused with the bra-ket notation of quantum mechanics which will not play any role in this paper.

${ }^{4}$ There are $n(n-3) / 2$ independent cross-ratios as long as the spacetime dimension $d$ is sufficiently large, more precisely $d+1 \geq n$. In lower number of dimensions, some of the cross-ratios defined in (1.6) become dependent, such that there are only $n d-(d+2)(d+1) / 2$ independent cross-ratios.

${ }^{5}$ The preferred appearance of $x_{1}, x_{n}$ in the choice of cross-ratios, and the related asymmetry between the external scaling dimensions $\Delta_{1}, \Delta_{n}$ and the remaining ones $\Delta_{2}, \ldots, \Delta_{n-1}$ in the power series expansion (1.1), arises naturally in the derivation of the $n$-point conformal block as a particular double-OPE limit of the holographic representation of an $(n+2)$-point conformal block, discussed in section 3 , which preferentially identifies $x_{1}$ and $x_{n}$.
} 
The conformal block is uniquely determine based on the following conditions [22]. They satisfy the multipoint conformal Casimir eigenvalue equations

$$
\begin{aligned}
\left(\mathcal{L}^{(1)}+\cdots+\right. & \left.\mathcal{L}^{(K)}\right)^{2} W_{\Delta_{\delta_{1}} ; \ldots ; \Delta_{\delta_{n-3}}}^{\Delta_{1}, \Delta_{2}, \ldots, \Delta_{n-1}, \Delta_{n}}\left(x_{1}, \ldots, x_{n}\right) \\
& =C_{2}\left(\Delta_{\delta_{K-1}}\right) W_{\Delta_{\delta_{1}} ; \ldots ; \Delta_{\delta_{n-3}}}^{\Delta_{1}, \Delta_{2}, \ldots, \Delta_{n-1}, \Delta_{n}}\left(x_{1}, \ldots, x_{n}\right)
\end{aligned}
$$

for all $2 \leq K \leq n-2$, where $\mathcal{L}_{A B}^{(i)}$ are the generators of the Euclidean conformal group $\mathrm{SO}(d+1,1)$, realized as differential operators built out of and acting on the coordinate $x_{i}$, with the quadratic Casimir operator defined as $\left(\mathcal{L}^{(i)}\right)^{2} \equiv \frac{1}{2} \mathcal{L}_{A B}^{(i)} \mathcal{L}^{A B(i)}$ (no sum over $i)$. The eigenvalues are given by $C_{2}(\Delta)=m_{\Delta}^{2}=\Delta(\Delta-d)$ for scalar exchange operators, which will be the case throughout this paper. Moreover, the blocks satisfy the following OPE limit

$$
\begin{aligned}
\lim _{x_{n} \rightarrow x_{n-1}} W_{\Delta_{\delta_{1}} ; \ldots ; \Delta_{\delta_{n-3}}}^{\Delta_{1}, \Delta_{2}, \ldots, \Delta_{n-1}, \Delta_{n}}\left(x_{1}, \ldots, x_{n}\right) \\
\quad=\left(x_{(n-1) n}^{2}\right)^{\Delta_{\delta_{n-3},(n-1) n}} W_{\Delta_{\delta_{1}} ; \ldots ; \Delta_{\delta_{n-4}}}^{\Delta_{1}, \Delta_{2}, \ldots, \Delta_{n-2}, \Delta_{\delta_{n-3}}}\left(x_{1}, \ldots, x_{n-1}\right),
\end{aligned}
$$

or pictorially,

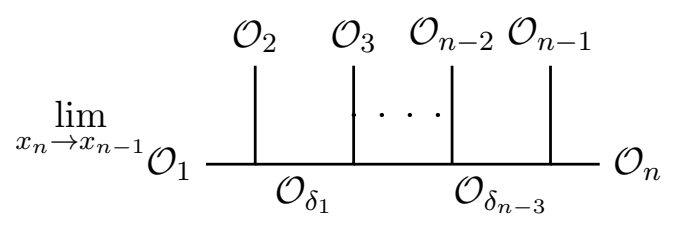

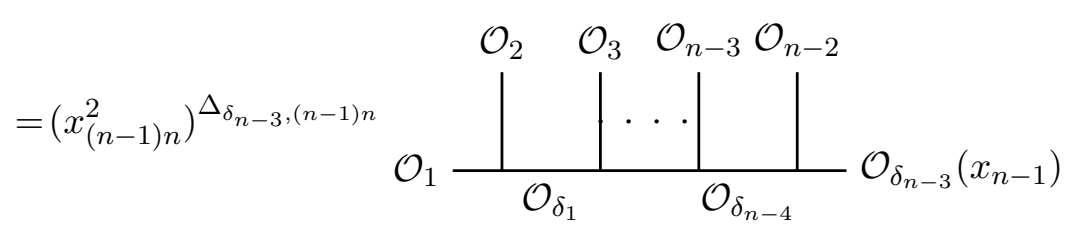

and symmetrically an analogous limit when $x_{2} \rightarrow x_{1}$. Further, the blocks have been normalized such that for $u_{i} \ll 1$ for all $1 \leq i \leq n-3$, and $w_{r ; s} \approx 1$ for all $2 \leq r \leq s \leq n-1$, the $n$-point block has the leading behavior

$$
W_{\Delta_{\delta_{1}} ; \ldots ; \Delta_{\delta_{n-3}}}^{\Delta_{1}, \ldots, \Delta_{n}}\left(x_{i}\right) \approx W_{0}^{(n)}\left(x_{i}\right)\left(\prod_{i=1}^{n-3} u_{i}^{\frac{\Delta_{\delta_{i}}}{2}}\right) .
$$

The final claim follows trivially from an alternate, rapidly convergent power series expansion of the conformal blocks presented in section 4 .

\section{Low-point examples of holographic duals}

In this section we provide the simplest non-trivial demonstration of the new techniques. First we will obtain the holographic dual of the six-point block in the comb channel, from which we shall recover the well-known purely boundary power series expansion of the fourpoint block. A second non-trivial example is provided in appendix B - it focuses on the holographic dual for the comb channel seven-point block, from which a power series expansion is obtained for the five-point block. In the next section, we will generalize these results to obtain the $n$-point comb channel conformal block. 


\subsection{Holographic dual of the six-point block}

Before discussing the six-point block, let us first establish some notation by reviewing recent results for the five-point block. In ref. [55] a holographic geodesic diagram representation was worked out for the $d$-dimensional global scalar five-point conformal block $W_{\Delta_{\delta_{1} ; \Delta_{\delta_{2}}}}^{\Delta_{1}, \ldots, \Delta_{5}}\left(x_{i}\right)$. Such a block corresponds to external scalar insertions of dimensions $\Delta_{1}, \ldots, \Delta_{5}$, and represents the contribution coming from the exchange of scalar representations (and their conformal families) labelled by dimensions $\Delta_{\delta_{1}}$ and $\Delta_{\delta_{2}}$. The precise relation is,

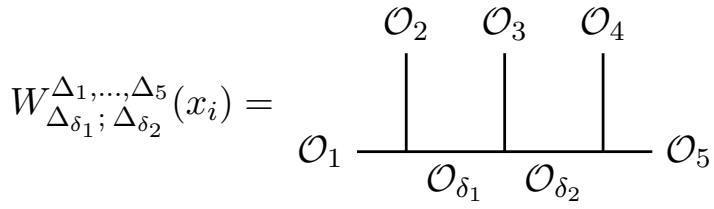

$$
\begin{aligned}
& =\frac{4}{B\left(\Delta_{\delta_{1} 1,2}, \Delta_{\delta_{1} 2,1}\right) B\left(\Delta_{\delta_{2} 4,5}, \Delta_{\delta_{2} 5,4}\right)} \mathcal{W}_{\Delta_{\delta_{1}} ; \Delta_{\delta_{2}}}^{\Delta_{1}, \ldots, \Delta_{5}}\left(x_{i}\right),
\end{aligned}
$$

where $B(s, t)=\Gamma(s) \Gamma(t) / \Gamma(s+t)$ is the Euler Beta function, and $\mathcal{W}$ is a linear combination of five-point geodesic diagrams (see figure 2 for notation and definition),

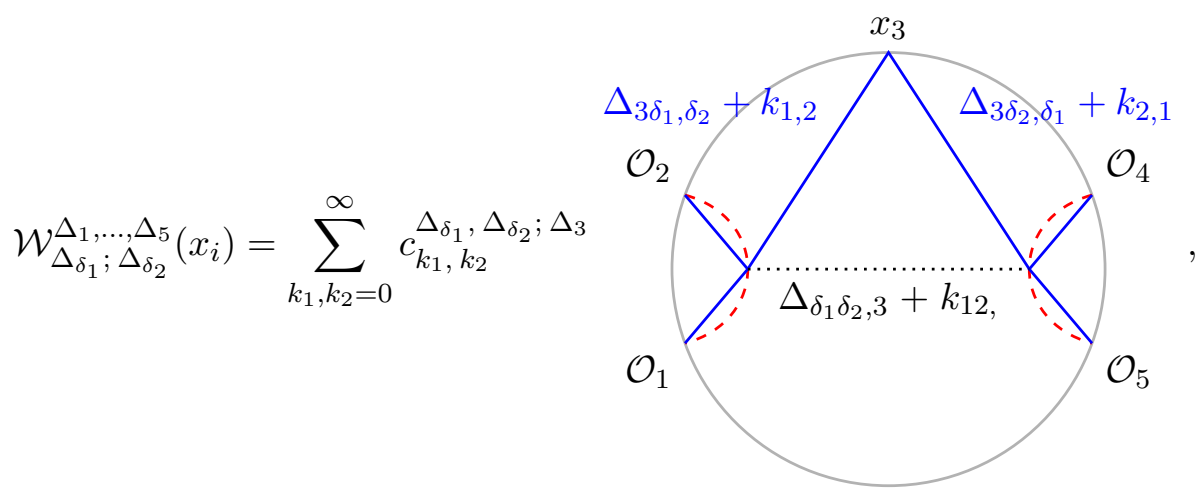

with the coefficients $c_{k_{1}, k_{2}}^{\Delta_{\delta_{1}}, \Delta_{\delta_{2}} ; \Delta_{3}}$ given by,

$$
\begin{aligned}
c_{k_{1}, k_{2}}^{\Delta_{\delta_{1}}, \Delta_{\delta_{2}} ; \Delta_{3}} \equiv & \frac{(-1)^{k_{1}+k_{2}}}{k_{1} ! k_{2} !} \frac{\left(1-\Delta_{3 \delta_{2}, \delta_{1}}\right)_{k_{1}}\left(1-\Delta_{3 \delta_{1}, \delta_{2}}\right)_{k_{2}}}{\left(\Delta_{\delta_{1}}-d / 2+1\right)_{k_{1}}\left(\Delta_{\delta_{2}}-d / 2+1\right)_{k_{2}}} \\
& \times\left(\Delta_{3 \delta_{1}, \delta_{2}}\right)_{k_{1,2}}\left(\Delta_{3 \delta_{2}, \delta_{1}}\right)_{k_{2,1}}\left(\Delta_{\delta_{1} \delta_{2}, 3}\right)_{k_{12},} \\
& \times{ }_{3} F_{2}\left[\left\{-k_{1},-k_{2}, \Delta_{\delta_{1} \delta_{2} 3}-d / 2\right\} ;\left\{\Delta_{3 \delta_{2}, \delta_{1}}-k_{1}, \Delta_{3 \delta_{1}, \delta_{2}}-k_{2}\right\} ; 1\right] .
\end{aligned}
$$

The geodesic bulk diagram in (2.2) is a generalization of the geodesic Witten diagrams first obtained in the case of the four-point block [43].

The (holographic representation of the) conformal block satisfies the Casimir equations (1.7) (for $n=5$ and $K=2,3$ ) and has the expected leading behavior in the two OPE limits. Later in this section we will obtain an alternate geodesic diagram representation for the five-point block involving a single geodesic integral. Moreover, in appendix B, we will provide a power series (purely CFT) representation of the five-point block obtained 


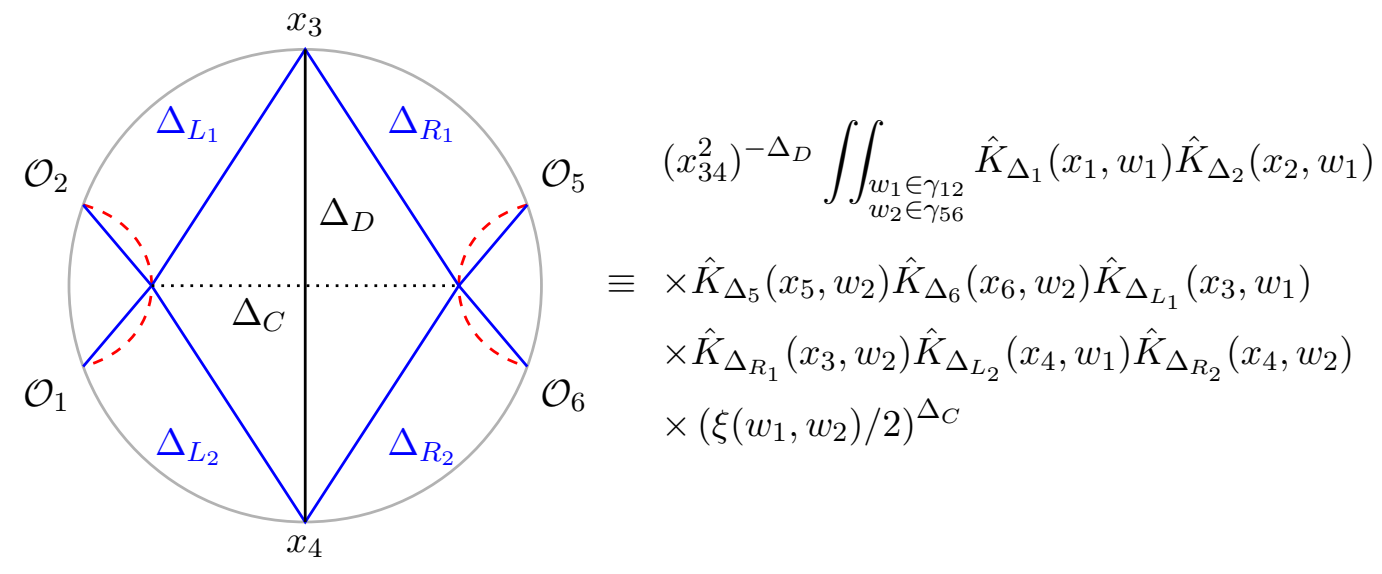

Figure 2. How to read comb-channel geodesic bulk diagrams. Geodesic bulk diagrams (also referred to as geodesic Witten diagrams) are AdS Feynman diagrams except with all bulk integrations restricted to boundary-anchored geodesics. Throughout this paper, boundary-anchored geodesics over which bulk points are to be integrated will be shown as red-dashed lines. In the diagram above, they represent the geodesics $\gamma_{12}$ and $\gamma_{56}$ joining $x_{1}$ to $x_{2}$ and $x_{5}$ to $x_{6}$, respectively. Bulkto-boundary propagators $\hat{K}_{\Delta}(x, z)$ will be shown with solid blue lines, and whenever the conformal dimension $\Delta$ associated with it is not clear from the figure, it will be mentioned explicitly. For example, in the six-point geodesic diagram above, the four bulk-to-boundary propagators incident on bulk points to be integrated over boundary anchored geodesics are associated with the conformal dimensions $\Delta_{i}$ of the operator insertions $\mathcal{O}_{i}$ as marked. The remaining four bulk-to-boundary propagators emanating from the coordinates $x_{3}$ and $x_{4}$ have conformal dimensions as displayed next to the blue lines. Unless stated otherwise, the operator $\mathcal{O}_{i}$ is understood to be located at boundary coordinate $x_{i}$. Solid black lines will refer to purely boundary contractions; for example in the diagram above the solid black line joining $x_{3}$ to $x_{4}$ corresponds to a factor of $\left(x_{34}^{2}\right)^{-\Delta_{D}}$. Finally dotted black lines will stand for factors of chordal distance $\left(\xi\left(w_{1}, w_{2}\right) / 2\right)^{\Delta}$ where $\xi\left(w_{1}, w_{2}\right)^{-1}=$ $\cosh \sigma\left(w_{1}, w_{2}\right)$ where $\sigma\left(w_{1}, w_{2}\right)$ is the geodesic distance between bulk points $w_{1}$ and $w_{2}$. We will be using the same propagator normalizations as in ref. [55]; see in particular [55, section 2] for the normalization of the bulk-to-boundary propagator as well as the relation between the bulk-to-bulk propagator and the chordal distance factor above.

by taking a so-called double-OPE limit of the holographic dual of the seven-point block. In the rest of this section, we illustrate this procedure for the case of the six-point block where we recover the four-point block in the double-OPE limit.

We first briefly discuss how to obtain the holographic representation of the six-point comb channel block. This discussion is schematic and light on technical details; we refer the reader to appendix A for computational details. The first step involves partially evaluating a particular AdS diagram involving cubic scalar couplings, in this case the diagram

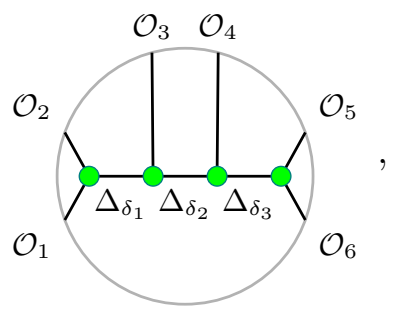


where all internal (green) cubic vertices are to be integrated over all of $\mathrm{AdS}_{d+1}$. In its direct-channel conformal block decomposition, the term corresponding to the exchange of three single-trace primaries takes the form

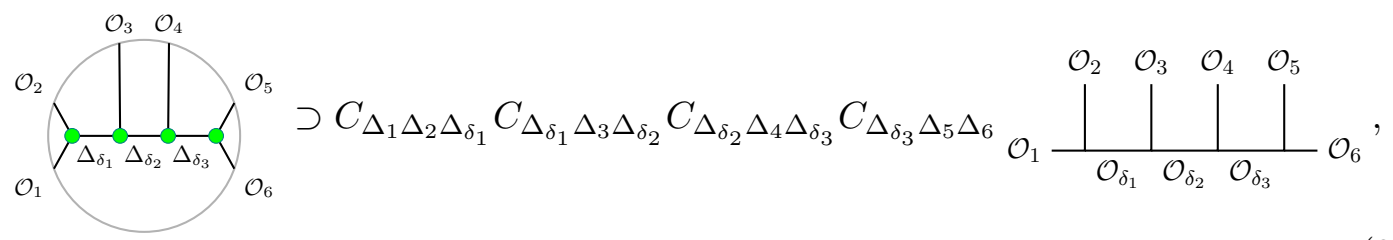

where $C_{\Delta_{i} \Delta_{j} \Delta_{k}}$ are the OPE coefficients associated with the dual generalized free-field CFT. This is the six-point conformal block we are after. The trick to extracting the block is to use the split representation for the central bulk-to-bulk propagator as well as certain powerful two-propagator [43] and three-propagator identities [56]. These propagator identities are especially helpful in evaluating AdS diagrams in a way which makes their direct-channel conformal block decomposition manifest $[43,56]$. Performing the remaining the boundary and spectral integrals arising from the split representation, one manifestly isolates precisely the term shown above, proportional to the right combination of OPE coefficients. (These steps are explicitly worked out in appendix A.) The object multiplying this factor of OPE coefficients produces a candidate for the holographic geodesic diagram representation of the block. Technically, the method outlined above is a heuristic derivation; the object obtained must be independently checked to be the claimed conformal block, for example via conformal Casimir equations. We will comment on certain interesting implications of this derivation in section 4 in the context of obtaining multipoint conformal blocks in arbitrary channels.

The procedure outlined above leads to the following geodesic diagram representation, which we claim to be the six-point conformal block in the comb channel,

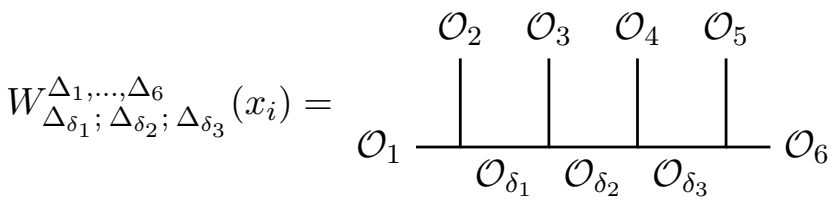

$$
\begin{aligned}
& =\frac{4}{B\left(\Delta_{\delta_{1} 1,2}, \Delta_{\delta_{1} 2,1}\right) B\left(\Delta_{\delta_{3} 5,6}, \Delta_{\delta_{3} 6,5}\right)} \mathcal{W}_{\Delta_{\delta_{1}} ; \Delta_{\delta_{2}} ; \Delta_{\delta_{3}}}^{\Delta_{1}, \ldots, \Delta_{6}}\left(x_{i}\right),
\end{aligned}
$$

where the $\mathcal{W}$ is a linear combination of six-point geodesic diagrams (see figure 2),

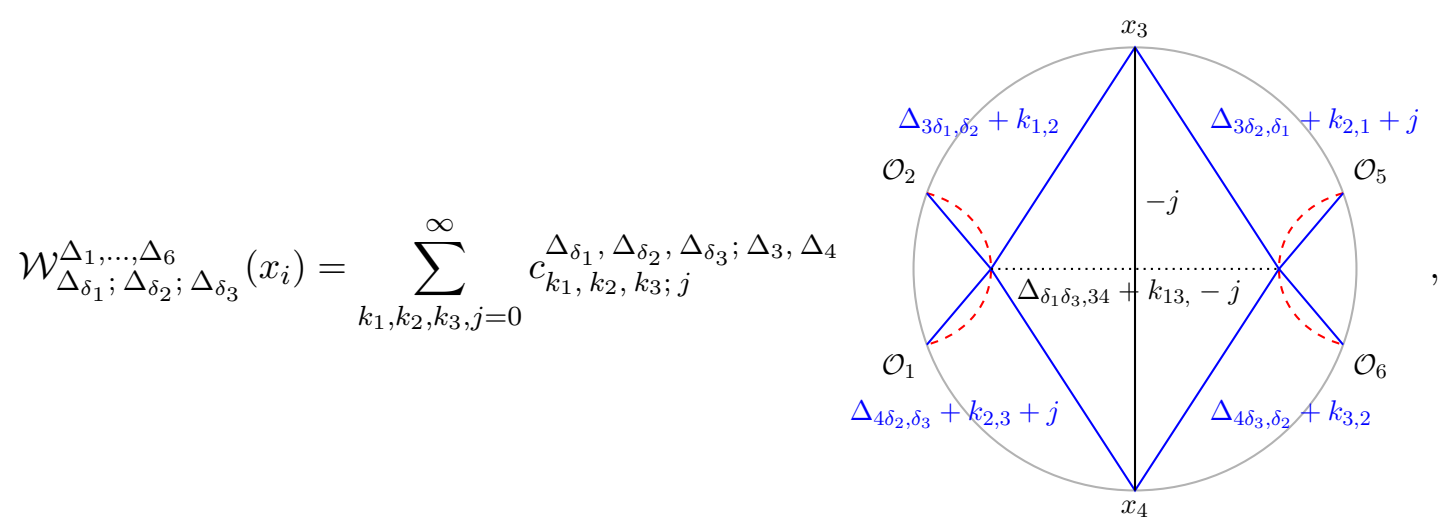


with the coefficients given by

$$
\begin{aligned}
& c_{k_{1}, k_{2},}^{\Delta_{\delta_{1}}, k_{3} ; j}, \Delta_{\delta_{3}} ; \Delta_{3}, \Delta_{4} \\
& \equiv \lambda_{6} \frac{(-1)^{k_{1}+k_{3}+j}}{k_{1} ! k_{2} ! k_{3} ! j !} \frac{\left(1-\Delta_{3 \delta_{2}, \delta_{1}}\right)_{k_{1}}\left(1-\Delta_{3 \delta_{1}, \delta_{2}}\right)_{k_{2}}\left(1-\Delta_{4 \delta_{3}, \delta_{2}}\right)_{k_{2}}\left(1-\Delta_{4 \delta_{2}, \delta_{3}}\right)_{k_{3}}}{\left(\Delta_{\delta_{1}}-d / 2+1\right)_{k_{1}}\left(\Delta_{\delta_{2}}-d / 2+1\right)_{k_{2}}\left(\Delta_{\delta_{3}}-d / 2+1\right)_{k_{3}}} \\
& \times\left(\Delta_{3 \delta_{1}, \delta_{2}}\right)_{k_{1,2}}\left(\Delta_{3 \delta_{2}, \delta_{1}}\right)_{k_{2,1}+j}\left(\Delta_{4 \delta_{2}, \delta_{3}}\right)_{k_{2,3}+j}\left(\Delta_{4 \delta_{3}, \delta_{2}}\right)_{k_{3,2}}\left(\Delta_{\delta_{1} \delta_{3}, 34}\right)_{k_{13,},-j} \\
& \times{ }_{3} F_{2}\left[\left\{-k_{1},-k_{2}, \Delta_{\delta_{1} \delta_{2} 3,}-d / 2\right\} ;\left\{\Delta_{3 \delta_{2}, \delta_{1}}-k_{1}, \Delta_{3 \delta_{1}, \delta_{2}}-k_{2}\right\} ; 1\right] \\
& \times{ }_{3} F_{2}\left[\left\{-k_{2},-k_{3}, \Delta_{\delta_{2} \delta_{3} 4,}-d / 2\right\} ;\left\{\Delta_{4 \delta_{3}, \delta_{2}}-k_{2}, \Delta_{4 \delta_{2}, \delta_{3}}-k_{3}\right\} ; 1\right],
\end{aligned}
$$

and

$$
\lambda_{6} \equiv \frac{\Gamma\left(1-\Delta_{\delta_{1} \delta_{2}, 3}\right) \Gamma\left(1-\Delta_{\delta_{2} \delta_{3}, 4}\right)}{\Gamma\left(1-\Delta_{\delta_{1} \delta_{3}, 34}\right) \Gamma\left(1-\Delta_{\delta_{2}}\right)} .
$$

It is worth pointing out the close structural similarity of the geodesic bulk diagram as well as the functional form of the coefficients with the five-point example. In particular, the five-point geodesic diagram had one "triangle" formed by two blue lines (bulk-to-boundary propagators) and a single dotted line (factor of chordal distance), and precisely one factor of the hypergeometric ${ }_{3} F_{2}$ function in the coefficient, while the six-point block has two triangles and two factors of ${ }_{3} F_{2} \cdot{ }^{6}$ We will return to this observation in section 4 .

To prove the claim above we need to show that the conformal block satisfies the right differential equations (1.7) (for $n=6, K=2,3,4$ ) with the right boundary conditions, expressed in terms of the OPE limits (1.8), reproduced below for convenience

$$
\begin{aligned}
& W_{\Delta_{\delta_{1}} ; \Delta_{\delta_{2}} ; \Delta_{\delta_{3}}}^{\Delta_{1}, \ldots, \Delta_{6}}\left(x_{1}, x_{2}, x_{3}, x_{4}, x_{5}, x_{6}\right) \stackrel{x_{2} \rightarrow x_{1}}{\longrightarrow}\left(x_{12}^{2}\right)^{\Delta_{\delta_{1}, 12}} W_{\Delta_{\delta_{2}} ; \Delta_{\delta_{3}}}^{\Delta_{\delta_{1}}, \Delta_{3}, \Delta_{4}, \Delta_{5}, \Delta_{6}}\left(x_{1}, x_{3}, x_{4}, x_{5}, x_{6}\right) \\
& W_{\Delta_{\delta_{1}} ; \Delta_{\delta_{2}} ; \Delta_{\delta_{3}}}^{\Delta_{1}, \ldots, \Delta_{6}}\left(x_{1}, x_{2}, x_{3}, x_{4}, x_{5}, x_{6}\right) \stackrel{x_{6} \rightarrow x_{5}}{\longrightarrow}\left(x_{56}^{2}\right)^{\Delta_{\delta_{3}, 56}} W_{\Delta_{\delta_{1}} ; \Delta_{\delta_{2}}}^{\Delta_{1}, \Delta_{2}, \Delta_{3}, \Delta_{4}, \Delta_{\delta_{3}}}\left(x_{1}, x_{2}, x_{3}, x_{4}, x_{5}\right) .
\end{aligned}
$$

The conformal Casimir check is set up in embedding space and the calculation proceeds identically to the conformal Casimir checks for the holographic representations of the fivepoint block [55] and the six-point block in the OPE channel [56]. Since no new ingredients are required, we refrain from including the somewhat lengthy albeit straightforward proof and simply point the reader to refs. $[55,56]$ for reference. ${ }^{7}$ In lieu of that, in the next section we will provide a conformal Casimir proof for the power series expansion of the six-point block which will be obtained later.

In the remainder of this section, we would like to focus on the OPE limits (2.10) of the six-point block (2.6)-(2.7). If this is indeed the six-point conformal block as claimed,

\footnotetext{
${ }^{6}$ The four-point case has no triangles and no factors of ${ }_{3} F_{2}$ in its double power series expansion.

${ }^{7}$ The Casimir check relies on a particular non-trivial functional identity obeyed by the hypergeometric function ${ }_{3} F_{2}$ which was also previously employed in the five-point case [55],

$$
E(D-B){ }_{3} F_{2}\left[\begin{array}{c}
A+1, B, C \\
D+1, E
\end{array} ; 1\right]+B(E-C){ }_{3} F_{2}\left[\begin{array}{c}
A+1, B+1, C \\
D+1, E+1
\end{array} ; 1\right]-D E_{3} F_{2}\left[\begin{array}{c}
A, B, C \\
D, E
\end{array} ; 1\right]=0 .
$$

The only difference is that due to two factors of the hypergeometric function in (2.8), this identity must now be applied twice, once for each factor.
} 
taking a single OPE limit should produce an (alternate) holographic representation for the five-point block involving a single geodesic integral, and taking a further OPE limit should reproduce the power series expansion of the four-point block purely in terms of boundary coordinates. We will utilize this strategy in the next section to go from the holographic representation of the $(n+2)$-point conformal block in the comb channel to produce an explicit power series expansion of the $n$-point comb channel block. For illustrative purposes, a seven-point to five-point block example is provided in appendix B.

\subsection{OPE limit of the six-point block}

Generically, all OPE limits in this paper will involve integrals of the following kind:

$$
\begin{aligned}
\lim _{x_{2} \rightarrow x_{1}} \int_{w \in \gamma_{12}} \hat{K}_{\Delta_{1}}\left(x_{1}, w\right) \hat{K}_{\Delta_{2}}\left(x_{2}, w\right)\left(\prod_{i=1}^{j} \hat{K}_{\Delta_{a_{i}}}\left(x_{a_{i}}, w\right)\right)\left(\prod_{i=1}^{k}\left(\frac{\xi\left(w_{b_{i}}, w\right)}{2}\right)^{\Delta_{b_{i}}}\right) \\
=\frac{B\left(\Delta_{a_{1} \ldots a_{j} b_{1} \ldots b_{k} 1,2}, \Delta_{a_{1} \ldots a_{j} b_{1} \ldots b_{k} 2,1}\right)}{2} \frac{\left(x_{12}^{2}\right)^{\Delta_{a_{1} \ldots a_{j} b_{1} \ldots b_{k}, 12}}}{\prod_{i=1}^{j}\left(x_{1 a_{i}}^{2}\right)^{\Delta_{a_{i}}}}\left(\prod_{i=1}^{k} \hat{K}_{\Delta_{b_{i}}}\left(x_{1}, w_{b_{i}}\right)\right)
\end{aligned}
$$

which is straightforward to derive (assuming convergence of the integral). In this section we focus on the OPE limit $x_{2} \rightarrow x_{1}$ of the six-point block. Due to the symmetry of the six-point block, we need only check one of the OPE limits in (2.10); the other one follows immediately from a simple relabeling. Using (2.12), we have

$$
W_{\Delta_{\delta_{1}} ; \Delta_{\delta_{2}} ; \Delta_{\delta_{3}}}^{\Delta_{1}, \ldots, \Delta_{6}}\left(x_{i}\right)
$$

We are interested in the leading behavior as $x_{2} \rightarrow x_{1}$, so we set the non-negative integral parameter $k_{1}=0$ above. Comparing with the first line of (2.10), it is sufficient to show that this holographic representation is indeed the five-point conformal block expected to 
be obtained in this limit, that is

$$
\begin{aligned}
& W_{\Delta_{\delta_{2}} ; \Delta_{\delta_{3}}}^{\Delta_{\delta_{1}}, \Delta_{3}, \Delta_{4}, \Delta_{5}, \Delta_{6}}\left(x_{1}, x_{3}, x_{4}, x_{5}, x_{6}\right) \\
& \stackrel{!}{=} \frac{2}{B\left(\Delta_{\delta_{3} 5,6}, \Delta_{\delta_{3} 6,5}\right)} \sum_{k_{2}, k_{3}, j=0}^{\infty} c_{0, k_{2}, k_{3} ; j}^{\Delta_{\delta_{1}}, \Delta_{\delta_{2}}, \Delta_{\delta_{3}} ; \Delta_{3}, \Delta_{4}} \\
& \Delta_{4 \delta_{2}, \delta_{3}}+k_{2,3}
\end{aligned}
$$

This is easily checked by showing that the r.h.s. above, which we call $V$, satisfies the differential equations and boundary conditions of a five-point block. Specifically,

$$
\begin{aligned}
\left(\mathcal{L}^{(1)}+\mathcal{L}^{(3)}\right)^{2} V & =C_{2}\left(\Delta_{\delta_{2}}\right) V \\
\left(\mathcal{L}^{(1)}+\mathcal{L}^{(3)}+\mathcal{L}^{(4)}\right)^{2} V & =C_{2}\left(\Delta_{\delta_{3}}\right) V
\end{aligned}
$$

with $V$ reducing to four-point blocks in the OPE limit,

$$
\begin{aligned}
& V \stackrel{x_{3} \rightarrow x_{1}}{\longrightarrow}\left(x_{13}^{2}\right)^{\Delta_{\delta_{2}, \delta_{1} 3}} W_{\Delta_{\delta_{3}}}^{\Delta_{\delta_{2}}, \Delta_{4}, \Delta_{5}, \Delta_{6}}\left(x_{1}, x_{4}, x_{5}, x_{6}\right) \\
& V \stackrel{x_{6} \rightarrow x_{5}}{\longrightarrow}\left(x_{56}^{2}\right)^{\Delta_{\delta_{3}, 56}} W_{\Delta_{\delta_{2}}}^{\Delta_{\delta_{1}}, \Delta_{3}, \Delta_{4}, \Delta_{\delta_{3}}}\left(x_{1}, x_{3}, x_{4}, x_{5}\right) .
\end{aligned}
$$

Just as discussed near the end of section 2.1, the conformal Casimir check (2.15) is once again straightforward to show using directly the techniques of ref. [55] and the functional identity (2.11). In the interest of keeping the length of this paper reasonable, we will refrain from presenting the technical details.

The OPE limits (2.16) are also straightforward to work out. Interestingly, the first OPE limit in (2.16) leads to the (holographic) geodesic bulk diagram representation of the four-point block, and the second furnishes a (boundary) power series expansion. We discuss these limits next.

\subsubsection{Recovering the four-point block from the six-point block}

Let's first discuss the limit $x_{3} \rightarrow x_{1}$. In this limit, using (2.12) we find that $V$ is proportional to $\left(x_{13}^{2}\right)^{\Delta_{\delta_{2}, \delta_{1} 3}+k_{2}}$ where $k_{2}$ is summed over non-negative integers. Thus the leading order 
contribution comes from setting $k_{2}=0$, which gives

$$
V \stackrel{x_{3} \rightarrow x_{1}}{\longrightarrow} \frac{2\left(x_{13}^{2}\right)^{\Delta_{\delta_{2}, \delta_{1} 3}}}{B\left(\Delta_{\delta_{3} 5,6}, \Delta_{\delta_{3} 6,5}\right)} \sum_{k_{3}, j=0}^{\infty} c_{0,0, k_{3} ; j}^{\Delta_{\delta_{1}}, \Delta_{\delta_{2}}, \Delta_{\delta_{3}} ; \Delta_{3}, \Delta_{4}}
$$

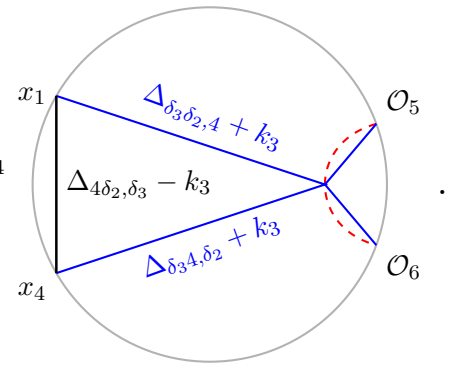

The free sum over $j$ is easily performed to give

$$
\begin{aligned}
& V \stackrel{x_{3} \rightarrow x_{1}}{\longrightarrow} \frac{2\left(x_{13}^{2}\right)^{\Delta_{\delta_{2}, \delta_{1} 3}}}{B\left(\Delta_{\delta_{3} 5,6}, \Delta_{\delta_{3} 6,5}\right)} \sum_{k_{3}=0}^{\infty} \frac{\left(\Delta_{\delta_{3} \delta_{2}, 4}\right)_{k_{3}}\left(\Delta_{\delta_{3} 4, \delta_{2}}\right)_{k_{3}}}{k_{3} !\left(\Delta_{\delta_{3}}-d / 2+1\right)_{k_{3}}} \\
& =\frac{4\left(x_{13}^{2}\right)^{\Delta_{\delta_{2}, \delta_{1} 3}}}{B\left(\Delta_{\delta_{3} 5,6}, \Delta_{\delta_{3} 6,5}\right) B\left(\Delta_{\delta_{3} \delta_{2}, 4}, \Delta_{\delta_{3} 4, \delta_{2}}\right)} \\
& \times \sum_{k_{3}=0}^{\infty} \frac{\left(\Delta_{\delta_{3}}\right)_{2 k_{3}}}{k_{3} !\left(\Delta_{\delta_{3}}-d / 2+1\right)_{k_{3}}} \\
& \mathcal{O}_{\delta_{2}}\left(x_{1}\right) \quad \mathcal{O}_{5}\left(x_{5}\right) \\
& \mathcal{O}_{4}\left(x_{4}\right) \\
& \Delta_{\delta_{3}}+2 k_{3} \\
& \mathcal{O}_{6}\left(x_{6}\right) \\
& =\left(x_{13}^{2}\right)^{\Delta_{\delta_{2}, \delta_{1} 3}} W_{\Delta_{\delta_{3}}}^{\Delta_{\delta_{2}}, \Delta_{4}, \Delta_{5}, \Delta_{6}}\left(x_{1}, x_{4}, x_{5}, x_{6}\right),
\end{aligned}
$$

where to get to the second equality we used ref. [55, eq. (A.4)] which re-expresses a particular combination of bulk-to-boundary propagators in the first equality as a geodesic integral. Performing the $k_{3}$ sum by using the relation between the chordal distance measure $\xi$ and the bulk-to-bulk propagator (see e.g. ref. [55, eq. (2.8)] for the precise relation in our conventions), one immediately recovers the original geodesic diagram representation of the four-point block [43].

Now let's consider the other OPE limit in (2.16). As $x_{6} \rightarrow x_{5}$, the leading contribution is given by

$$
V \stackrel{x_{6} \rightarrow x_{5}}{\longrightarrow}\left(x_{56}^{2}\right)^{\Delta_{\delta_{3}, 56}} \sum_{k_{2}, j=0}^{\infty} c_{0, k_{2}, 0 ; j}^{\Delta_{\delta_{1}}, \Delta_{\delta_{2}}, \Delta_{\delta_{3}} ; \Delta_{3}, \Delta_{4}}
$$

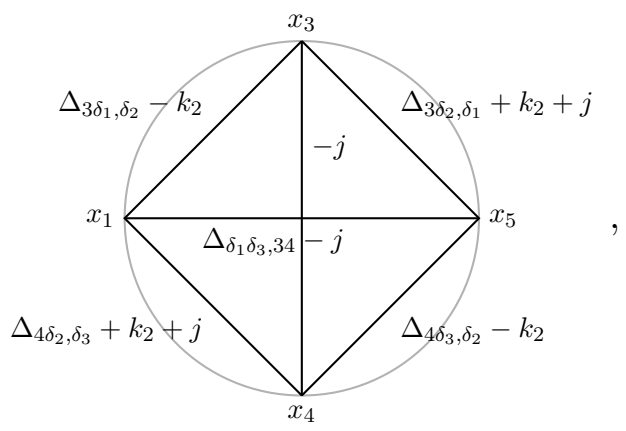


with the subleading contributions suppressed by higher positive powers of $x_{56}^{2}$. Recalling from figure 2 that lines joining points on the boundary of the Poincaré disk represent factors of the form $\left(x_{34}^{2}\right)^{j}$ etc., this limit can be written explicitly as

$$
V \stackrel{x_{6} \rightarrow x_{5}}{\longrightarrow} \frac{\left(x_{56}^{2}\right)^{\Delta_{\delta_{3}, 56}}}{\left(x_{13}^{2}\right)^{\Delta_{\delta_{1} 3},}\left(x_{45}^{2}\right)^{\Delta_{4 \delta_{3}}}}\left(\frac{x_{35}^{2}}{x_{15}^{2}}\right)^{\Delta_{\delta_{1}, 3}}\left(\frac{x_{15}^{2}}{x_{14}^{2}}\right)^{\Delta_{4, \delta_{3}}} u^{\frac{\Delta_{\delta_{2}}}{2}} \sum_{k_{2}, j=0}^{\infty} c_{0, k_{2}, 0 ; j}^{\Delta_{\delta_{1}}, \Delta_{\delta_{2}}, \Delta_{\delta_{3}} ; \Delta_{3}, \Delta_{4}} u^{k_{2}} v^{j}
$$

where the conformal cross-ratios are defined to be

$$
u \equiv \frac{x_{13}^{2} x_{45}^{2}}{x_{14}^{2} x_{35}^{2}} \quad v \equiv \frac{x_{15}^{2} x_{34}^{2}}{x_{14}^{2} x_{35}^{2}} .
$$

Up to the expected overall factor of $\left(x_{56}^{2}\right)^{\Delta_{\delta_{3}, 56}},(2.20)$ is of the same form as (1.1) except for a different set of coordinate labels and conformal dimensions. More precisely,

$$
V \stackrel{x_{6} \rightarrow x_{5}}{\longrightarrow}\left(x_{56}^{2}\right)^{\Delta_{\delta_{3}, 56}} W_{\Delta_{\delta_{2}}}^{\Delta_{\delta_{1}}, \Delta_{3}, \Delta_{4}, \Delta_{\delta_{3}}}\left(x_{1}, x_{3}, x_{4}, x_{5}\right),
$$

in the notation described after (1.1). To show that (2.20) is indeed the power series expansion of the global four-point block as claimed, it helps to bring it to a more familiar form, by rewriting the series expansion in terms of powers of $(1-v),{ }^{8}$

$$
\begin{aligned}
V \stackrel{x_{6} \rightarrow x_{5}}{\longrightarrow} & \left(x_{56}^{2}\right)^{\Delta_{\delta_{3}, 56}} W_{0}^{\Delta_{\delta_{1}}, \Delta_{3}, \Delta_{4}, \Delta_{\delta_{3}}}\left(x_{1}, x_{3}, x_{4}, x_{5}\right) \\
& \times u^{\frac{\Delta_{\delta_{2}}}{2}} \sum_{k_{2}, \ell=0}^{\infty} \frac{u^{k_{2}}(1-v)^{\ell}}{k_{2} ! \ell !} \frac{\left(\Delta_{\delta_{2} \delta_{1}, 3}\right)_{k_{2}}\left(\Delta_{\delta_{2} \delta_{3}, 4}\right)_{k_{2}}}{\left(\Delta_{\delta_{2}}-d / 2+1\right)_{k_{2}}} \frac{\left(\Delta_{\delta_{2} 3, \delta_{1}}\right)_{k_{2}+\ell}\left(\Delta_{\delta_{2} 4, \delta_{3}}\right)_{k_{2}+\ell}}{\left(\Delta_{\delta_{2}}\right)_{2 k_{2}+\ell}},
\end{aligned}
$$

where the leg-factor $W_{0}$ was defined in (1.5). Up to the overall factor of $\left(x_{56}^{2}\right)^{\Delta_{\delta_{3}, 56}}$, this is precisely the power series expansion of the appropriate global scalar four-point block [12], thus confirming the second line of (2.16).

To conclude, we emphasize the main results of this section: We obtained and verified a holographic representation of the six-point block in the comb channel (2.6)-(2.9), and in the double-OPE limit $x_{2} \rightarrow x_{1}, x_{n} \rightarrow x_{n-1}$ for $n=6$, we recovered the explicit power series expansion of the four-point block (2.19). In appendix B, we provide another example of this - the holographic dual of the seven-point block leading to the power series expansion of the five-point block. In the next section, we will generalize this result to obtain the power series expansion of the $n$-point comb channel block from a similar double-OPE limit of the holographic dual of the $(n+2)$-point comb channel block.

${ }^{8}$ The standard trick to do that is as follows: We expand

$$
v^{j}=(1+v-1)^{j}=\sum_{\ell=0}^{j}\left(\begin{array}{l}
j \\
\ell
\end{array}\right)(-1)^{\ell}(1-v)^{\ell} .
$$

Then extending the upper limit of the binomial sum above to infinity with impunity and switching the order of $\ell$ and $j$ sums, perform the summation over $j$ in (2.20) to obtain a power series expansion in powers of $(1-v)$. 


\section{Multipoint block in the comb channel}

\subsection{Holographic dual of the $(n+2)$-point block and its double-OPE limit}

The low-point examples of five- and six-point blocks in the previous section, along with the seven-point example in appendix B allow us to make a guess for the holographic representation of any multipoint block in the comb channel. We conjecture the holographic representation of the $(n+2)$-point comb channel block $(n \geq 3)$, labelled as follows,

$$
\begin{aligned}
& \begin{array}{llllll}
\mathcal{O}_{e_{2}}\left(x_{1}\right) & \mathcal{O}_{2} & \mathcal{O}_{3} & \mathcal{O}_{n-2} & \mathcal{O}_{n-1} & \mathcal{O}_{e_{3}}\left(x_{n}\right)
\end{array}
\end{aligned}
$$

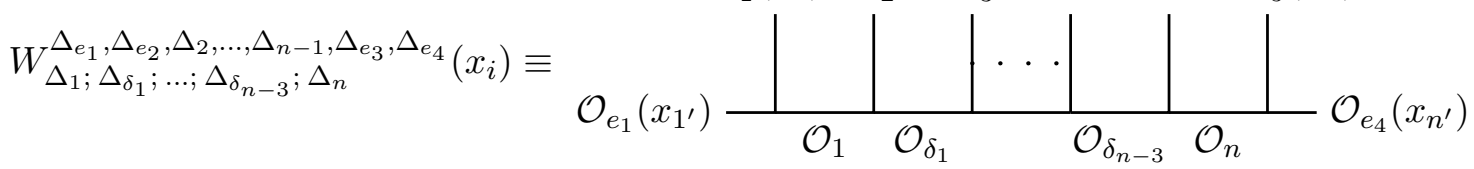

$$
\begin{aligned}
& =\frac{4 \mathcal{W}_{\Delta_{1} ; \Delta_{\delta_{1}} ; \ldots ; \Delta_{\delta_{n-3}} ; \Delta_{n}}^{\Delta_{e_{1}, \Delta_{e_{2}}, \Delta_{2}, \ldots, \Delta_{n-1}, \Delta_{e_{3}}, \Delta_{e_{4}}}}\left(x_{i}\right)}{B\left(\Delta_{1 e_{1}, e_{2}}, \Delta_{1 e_{2}, e_{1}}\right) B\left(\Delta_{n e_{3}, e_{4}}, \Delta_{n e_{4}, e_{3}}\right)},
\end{aligned}
$$

to be given by $\mathcal{W}$, which is the following linear combination of geodesic bulk diagrams (see figure 2 for the graphical notation and below for more notes),

$$
\begin{aligned}
& \mathcal{W}_{\Delta_{1} ; \Delta_{\delta_{1}} ; \ldots ; \Delta_{\delta_{n-3}} ; \Delta_{n}}^{\Delta_{e_{1}}, \Delta_{e_{2}}, \Delta_{2}, \ldots, \Delta_{n-1}, \Delta_{e_{3}}, \Delta_{e_{4}}}\left(x_{i}\right) \\
& =\sum_{\substack{k_{0}, k_{1}, k_{2}, \ldots, k_{n-3}, k_{n-2}, j_{\langle 2 \mid 3\rangle}, j_{\langle 2 \mid 4\rangle}, \ldots, j_{\langle n-2 \mid n-1\rangle}=0}}^{\infty} \begin{array}{c}
\Delta_{1}, \Delta_{\delta_{1}}, \Delta_{\delta_{2}}, \ldots, \Delta_{\delta_{n-3}}, \Delta_{n} ; \Delta_{2}, \Delta_{3}, \ldots, \Delta_{n-1} \\
c_{k_{2}}, k_{2}, \ldots, k_{n-3}, k_{n-2} ; j_{\langle 2 \mid 3\rangle}, j_{\langle 2 \mid 4\rangle}, \ldots, j_{\langle n-2 \mid n-1\rangle}
\end{array} \\
& \prod_{\mathcal{O}_{e_{1}}\left(x_{1^{\prime}}\right)}^{\mathcal{O}_{e_{3}}\left(x_{n}\right)}
\end{aligned}
$$

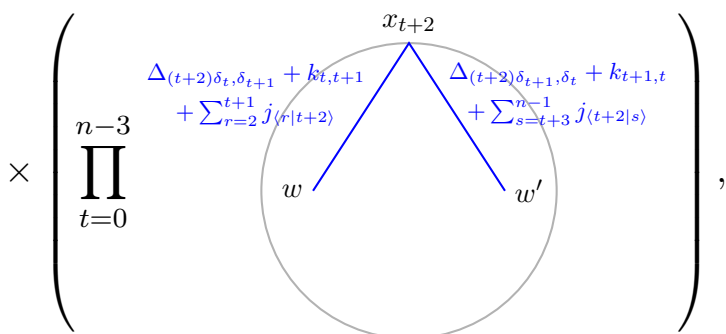

where the indices $\langle r \mid s\rangle$ represent the $\left(\begin{array}{c}n-2 \\ 2\end{array}\right)$ values the subscript on $j$ can take. The coefficients are given by (for $n \geq 3$ )

$$
\begin{aligned}
& c_{k_{0}, k_{1}, \ldots, k_{n-3}, k_{n-2} ; j_{\langle 2 \mid 3\rangle}, j_{\langle 2 \mid 4\rangle}, \ldots, j_{\langle n-2 \mid n-1\rangle}}^{\Delta_{1}, \Delta_{\delta_{1}}, \ldots, \Delta_{\delta_{n-3}}, \Delta_{n} ; \Delta_{2}, \ldots, \Delta_{n-1}} \equiv \lambda_{n+2}\left(\prod_{2 \leq r<s \leq n-1} \frac{(-1)^{j_{\langle r \mid s\rangle}}}{j_{\langle r \mid s\rangle} !}\right)\left(\prod_{i=0}^{n-2} \frac{1}{k_{i} !}\right) \\
& \quad \times\left(\prod_{i=1}^{n-3} \frac{\left(1-\Delta_{(i+1) \delta_{i-1}, \delta_{i}}\right)_{k_{i}}\left(1-\Delta_{(i+2) \delta_{i+1}, \delta_{i}}\right)_{k_{i}}}{\left(\Delta_{\delta_{i}}-d / 2+1\right)_{k_{i}}}\right) \frac{\left(1-\Delta_{2 \delta_{1}, 1}\right)_{k_{0}}\left(1-\Delta_{(n-1) \delta_{n-3}, n}\right)_{k_{n-2}}}{\left(\Delta_{1}-d / 2+1\right)_{k_{0}}\left(\Delta_{n}-d / 2+1\right)_{k_{n-2}}}
\end{aligned}
$$




$$
\begin{aligned}
& \times(-1)^{k_{0}+k_{n-2}}\left(\Delta_{1 n, 2 \ldots(n-1)}\right)_{k_{0(n-2),},-\sum_{2 \leq r<s \leq n-1} j_{\langle r \mid s\rangle}} \\
& \times\left(\prod_{t=0}^{n-3}\left(\Delta_{(t+2) \delta_{t}, \delta_{t+1}}\right)_{k_{t, t+1}+\sum_{r=2}^{t+1} j_{\langle r \mid t+2\rangle}}\left(\Delta_{(t+2) \delta_{t+1}, \delta_{t}}\right)_{k_{t+1, t}+\sum_{s=t+3}^{n-1} j_{\langle t+2 \mid s\rangle}}\right. \\
& \left.\times{ }_{3} F_{2}\left[\left\{-k_{t},-k_{t+1}, \Delta_{\delta_{t} \delta_{t+1}(t+2),}-\frac{d}{2}\right\} ;\left\{\Delta_{(t+2) \delta_{t+1}, \delta_{t}}-k_{t}, \Delta_{(t+2) \delta_{t}, \delta_{t+1}}-k_{t+1}\right\} ; 1\right]\right),
\end{aligned}
$$

with

$$
\lambda_{n+2} \equiv \frac{\prod_{t=0}^{n-3} \Gamma\left(1-\Delta_{\delta_{t} \delta_{t+1},(t+2)}\right)}{\Gamma\left(1-\Delta_{1 n, 2 \ldots(n-1)}\right) \prod_{i=1}^{n-3} \Gamma\left(1-\Delta_{\delta_{i}}\right)},
$$

and the additional definitions

$$
\Delta_{\delta_{0}} \equiv \Delta_{1} \quad \Delta_{\delta_{n-2}} \equiv \Delta_{n}
$$

A few remarks are in order regarding the conjecture for the $(n+2)$-point block above:

- For clarity we have split up a single geodesic bulk diagram in (3.2) into a chain of constituent factors. The first factor should be familiar to the reader from the geodesic diagram representation of a four-point block [43] (see also (2.18)). The second factor, corresponding to $\left(\begin{array}{c}n-2 \\ 2\end{array}\right)$ contractions (in fact, a perfect graph) between boundary points $x_{2}, \ldots, x_{n-1}$, appears in the holographic dual for all $n \geq 4$ (i.e. for the six-point block and higher). The third factor, which represents a product over $(n-2)$ pairs of bulk-to-boundary propagators, is present in the holographic dual for all $n \geq 3$ (i.e. five-point block and higher).

- All these constituent factors are to be merged together and should be understood as having been drawn on the same Poincaré disk. The bulk points $w, w^{\prime}$, which are to be integrated over the two boundary anchored geodesics have been marked explicitly to emphasize that the bulk-to-boundary propagators are incident on precisely the same bulk points; thus there are only two geodesic integrals to be performed.

- It is straightforward to check that this conjecture reduces to the already established holographic duals for the five-point block (2.1)-(2.3), the six-point block (2.6)-(2.9), and the seven-point block (B.1)-(B.4).

If this conjecture is true, its double-OPE limit, $x_{1^{\prime}} \rightarrow x_{1}, x_{n^{\prime}} \rightarrow x_{n}$ should lead to the $n$-point block (for $n \geq 4$ ) described in figure 1, up to some expected overall scaling factors,

$$
\begin{aligned}
& \lim _{\substack{x_{1^{\prime} \rightarrow x} \rightarrow x_{1} \\
x_{n^{\prime}} \rightarrow x_{n}}} W_{\Delta_{1} ; \Delta_{\delta_{1}} ; \ldots ; \Delta_{\delta_{n-3}} ; \Delta_{n}}^{\Delta_{e_{1}}, \Delta_{e_{2}}, \Delta_{2}, \ldots, \Delta_{n-1}, \Delta_{e_{3}}, \Delta_{e_{4}}}\left(x_{1^{\prime}}, x_{1}, \ldots, x_{n}, x_{n^{\prime}}\right) \\
& =\left(x_{11^{\prime}}^{2}\right)^{\Delta_{e_{1} e_{2}, 1}}\left(x_{n n^{\prime}}^{2}\right)^{\Delta_{e_{3} e_{4}, n}} W_{\Delta_{\delta_{1}} ; \ldots ; \Delta_{\delta_{n-3}}}^{\Delta_{1}, \Delta_{2}, \ldots, \Delta_{n-1}, \Delta_{n}}\left(x_{1}, \ldots, x_{n}\right),
\end{aligned}
$$


where, using (2.12), ${ }^{9}$ the explicit representation can be worked out to be (for $n \geq 4$ )

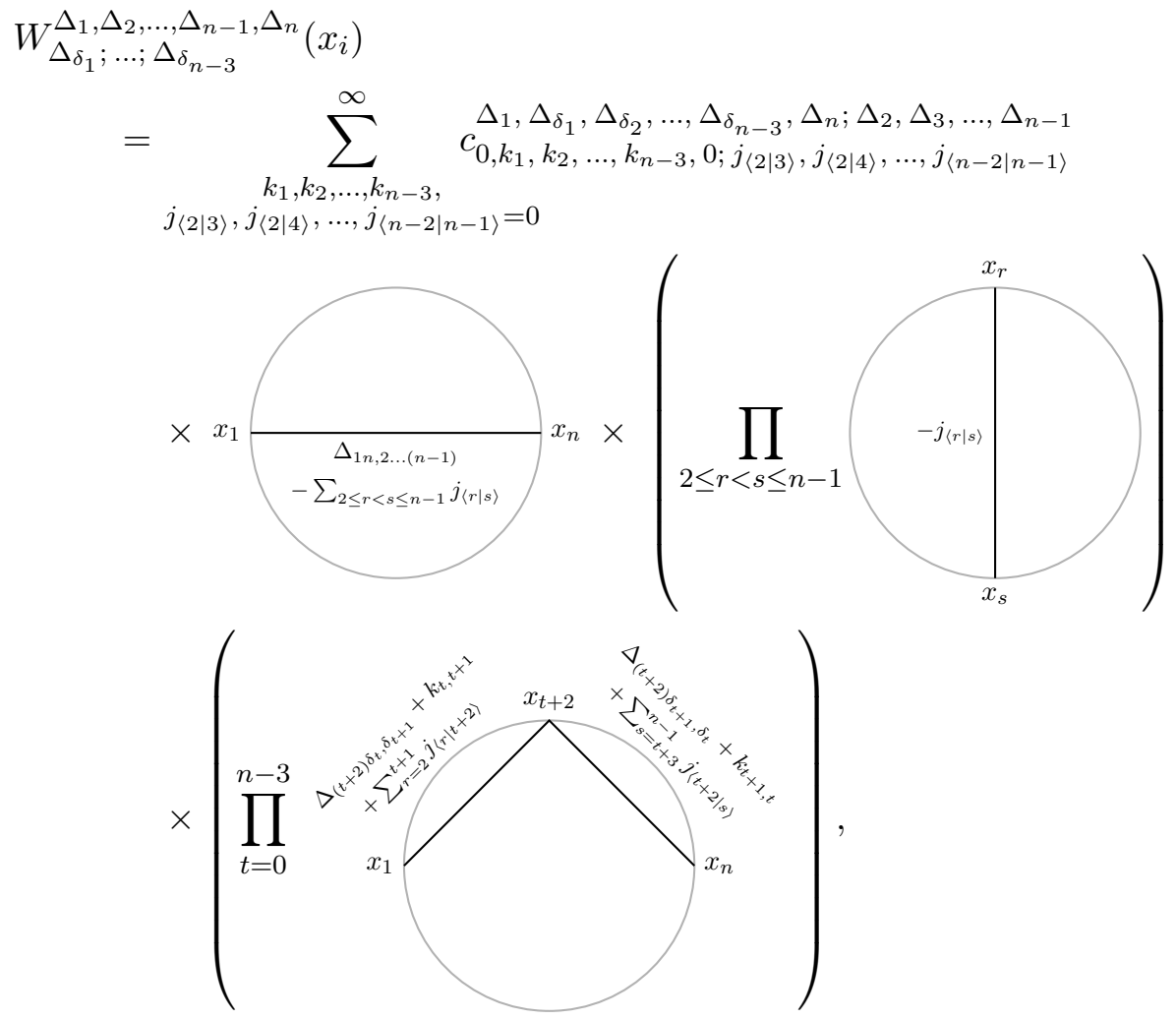

where we now additionally impose the identifications (1.4). Once again the chain of Poincaré disks above is interpreted as explained for the geodesic diagram representation. The coefficients themselves simplify to (for $n \geq 4$ ),

$$
\begin{aligned}
& c_{0, k_{1}, \ldots, k_{n-3}, 0 ; j_{\langle 2 \mid 3\rangle}, j_{\langle 2 \mid 4\rangle}, \ldots, j_{\langle n-2 \mid n-1\rangle}}^{\Delta_{1}, \Delta_{\delta_{1}}, \ldots, \Delta_{\delta_{n-3}}, \Delta_{n} ; \Delta_{2}, \ldots, \Delta_{n-1}}=\lambda_{n+2}\left(\prod_{2 \leq r<s \leq n-1} \frac{(-1)^{\left.j_{\langle r \mid s\rangle}\right\rangle}}{j_{\langle r \mid s\rangle} !}\right)\left(\prod_{i=1}^{n-3} \frac{1}{k_{i} !}\right) \\
& \times\left(\Delta_{1 n, 2 \ldots(n-1)}\right)_{-\sum_{2 \leq r<s \leq n-1} j_{\langle r \mid s\rangle}}\left(\prod_{t=0}^{n-3} \frac{\left(1-\Delta_{(t+1) \delta_{t-1}, \delta_{t}}\right)_{k_{t}}\left(1-\Delta_{(t+2) \delta_{t+1}, \delta_{t}}\right)_{k_{t}}}{\left(\Delta_{\delta_{t}}-d / 2+1\right)_{k_{t}}}\right. \\
& \times\left(\Delta_{(t+2) \delta_{t}, \delta_{t+1}}\right)_{k_{t, t+1}+\sum_{r=2}^{t+1} j_{\langle r \mid t+2\rangle}}\left(\Delta_{(t+2) \delta_{t+1}, \delta_{t}}\right)_{k_{t+1, t}+\sum_{s=t+3}^{n-1} j_{\langle t+2 \mid s\rangle}} \\
& \left.\times{ }_{3} F_{2}\left[\left\{-k_{t},-k_{t+1}, \Delta_{\delta_{t} \delta_{t+1}(t+2),}-\frac{d}{2}\right\} ;\left\{\Delta_{(t+2) \delta_{t+1}, \delta_{t}}-k_{t}, \Delta_{(t+2) \delta_{t}, \delta_{t+1}}-k_{t+1}\right\} ; 1\right]\right),
\end{aligned}
$$

where $\lambda_{n+2}$ is given in (3.4) and we employed (1.4) to write the coefficient above compactly. ${ }^{10}$

\footnotetext{
${ }^{9}$ In particular, the integer parameters $k_{0}$ and $k_{n-2}$ are set to zero to obtain the leading contribution on the r.h.s. of (3.6).

${ }^{10}$ For example, for $n=4$ one obtains the simplified coefficients

$$
c_{0, k_{1}, 0 ; j_{\langle 2 \mid 3\rangle}, \Delta_{1}, \Delta_{\delta_{1}}, \Delta_{4} ; \Delta_{2}, \Delta_{3}}^{\Delta_{2}}=\lambda_{6} \frac{(-1)^{j_{\langle 2 \mid 3\rangle}}}{j_{\langle 2 \mid 3\rangle} !} \frac{1}{k_{1} !} \frac{\left(\Delta_{2 \delta_{1}, 1}\right)_{k_{1}+j_{\langle 2 \mid 3\rangle}}\left(\Delta_{3 \delta_{1}, 4}\right)_{k_{1}+j_{\langle 2 \mid 3\rangle}}\left(\Delta_{14,23}\right)_{-j_{\langle 2 \mid 3\rangle}}}{\left(\Delta_{\delta_{1}}-d / 2+1\right)_{k_{1}}} .
$$


The coordinate dependence of the putative conformal block is captured pictorially in (3.7). Recalling the notation from figure 2, one can easily convert this to conformal cross-ratios as follows,

$$
\begin{aligned}
W_{\Delta_{\delta_{1}} ; \ldots ; \Delta_{\delta_{n-3}}}^{\Delta_{1}, \ldots, \Delta_{n}}\left(x_{i}\right)= & W_{0}^{(n)}\left(x_{i}\right)\left(\prod_{i=1}^{n-3} u_{i}^{\frac{\Delta_{\delta_{i}}}{2}}\right) \\
& \times \sum_{\substack{j_{\langle 2 \mid 3\rangle}, j_{\langle 2 \mid 4\rangle}, \ldots, k_{n-3}, k_{\langle n-2 \mid n-1\rangle}=0}}^{\infty} c_{0, k_{1}, \ldots, k_{n-3}, 0 ; j_{\langle 2 \mid 3\rangle}, j_{\langle 2 \mid 4\rangle}, \ldots, j_{\langle n-2 \mid n-1\rangle}}^{\Delta_{1}, \Delta_{\delta_{1}}, \ldots, \Delta_{\delta_{n-3}}, \Delta_{n} ; \Delta_{2}, \ldots, \Delta_{n-1}} \\
& \times\left(\prod_{i=1}^{n-3} u_{i}^{k_{i}}\right)\left(\prod_{2 \leq r<s \leq n-1} w_{r ; s}^{j_{\langle r \mid s\rangle}}\right)
\end{aligned}
$$

where the "leg factor" $W_{0}^{(n)}\left(x_{i}\right)$ is given by (1.5) and the cross-ratios $u_{i}, w_{r ; s}$ are defined in (1.6). This is precisely the power series expansion (1.1). ${ }^{11}$

While we do not directly prove the conjecture (3.1)-(3.3) for the holographic representation of the $(n+2)$-point block as this is not the main focus of this paper, in the remainder of this section, we will prove via conformal Casimir equations (1.7)-(1.8) that (3.10) is indeed the desired power series expansion of the $n$-point comb channel block.

\subsection{OPE limit of the $n$-point block}

Let's first verify the boundary conditions. Due to the symmetrical nature of the conjectural conformal block (3.7), we need only check one of the two OPE limits. We choose to work out the limit $x_{n} \rightarrow x_{n-1}$. It is easily gleaned from the diagrammatic representation (3.7) that the power series expansion is proportional to a factor of $\left(x_{(n-1) n}^{2}\right)^{k_{n-3}-\Delta_{(n-1) n, \delta_{n-3}} \text {, so }}$ we set $k_{n-3}=0$ to obtain the leading contribution in the OPE limit. It is straightforward

${ }^{11}$ Alternately, one can write it as

$$
\begin{aligned}
& W_{\Delta_{\delta_{1}} ; \ldots ; \Delta_{\delta_{n-3}}}^{\Delta_{1}, \ldots, \Delta_{n}}\left(x_{i}\right)=W_{0}^{(n)}\left(x_{i}\right)\left(\prod_{i=1}^{n-3} u_{i}^{\frac{\Delta_{\delta_{i}}}{2}}\right) \\
& \times \sum_{\substack{k_{1}, \ldots, k_{n-3}, j_{\langle 2 \mid 3\rangle}, j_{\langle 2 \mid 4\rangle}, \ldots, j_{\langle n-2 \mid n-1\rangle}=0}}^{\infty} \begin{array}{c}
\Delta_{1}, \Delta_{\delta_{1}}, \ldots, \Delta_{\delta_{n-3}}, \Delta_{n} ; \Delta_{2}, \ldots, \Delta_{n-1} \\
c_{0, k_{1}, \ldots, k_{n-3}, 0 ; j_{\langle 2 \mid 3\rangle}, j_{\langle 2 \mid 4\rangle}, \ldots, j_{\langle n-2 \mid n-1\rangle}}
\end{array} \\
& \times\left(\prod_{i=1}^{n-3} u_{i}^{k_{i}} v_{i}^{j^{\langle i+1 \mid i+2\rangle}}\right)\left(\prod_{\substack{2 \leq r<s \leq n-1 \\
s \neq r+1}} w_{r ; s}^{j_{r r|s\rangle}}\right),
\end{aligned}
$$

where we disallowed $s=r+1$ in the $w_{r ; s}$ cross-ratios, and collect them separately into the cross-ratios

$$
v_{i} \equiv w_{i+1 ; i+2}=\frac{x_{1 n}^{2} x_{(i+1)(i+2)}^{2}}{x_{(i+1) n}^{2} x_{1(i+2)}^{2}} \quad 1 \leq i \leq n-3 .
$$


to work out the full expansion,

$$
\begin{aligned}
& \lim _{x_{n} \rightarrow x_{n-1}} W_{\Delta_{\delta_{1}} ; \ldots ; \Delta_{\delta_{n-3}}}^{\Delta_{1}, \ldots, \Delta_{n}}\left(x_{i}\right) \\
& \rightarrow\left(x_{(n-1) n}^{2}\right)^{\Delta_{\delta_{n-3},(n-1) n}} \sum_{\substack{k_{1}, k_{2}, \ldots, k_{n-4}, j_{\langle 2 \mid 3\rangle}, j_{\langle 2 \mid 4\rangle}, \ldots, j_{\langle n-2 \mid n-1\rangle}=0}}^{\infty} \begin{array}{c}
\Delta_{1}, \Delta_{\delta_{1}}, \Delta_{\delta_{2}}, \ldots, \Delta_{\delta_{n-3}}, \Delta_{n} ; \Delta_{2}, \Delta_{3}, \ldots, \Delta_{n-4}, 0,0 ; j_{\langle 2 \mid 3\rangle}, j_{\langle 2 \mid 4\rangle}, \ldots, j_{\langle n-2 \mid n-1\rangle} \\
j_{\langle n}, k_{n-1}
\end{array}
\end{aligned}
$$

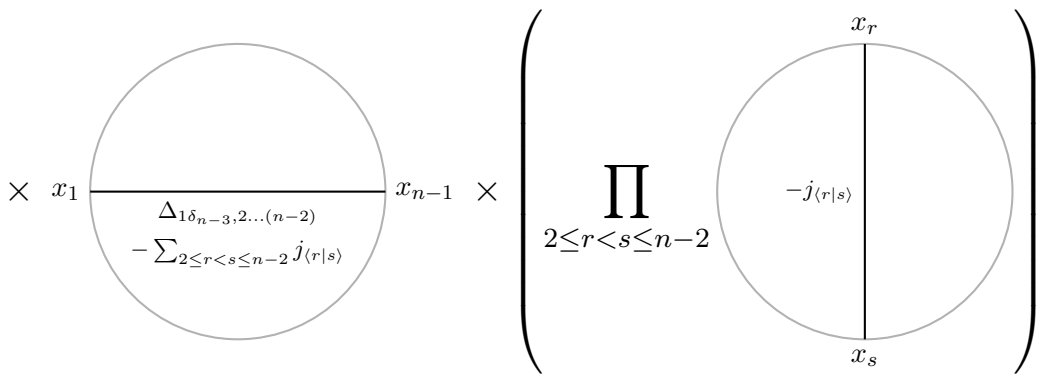

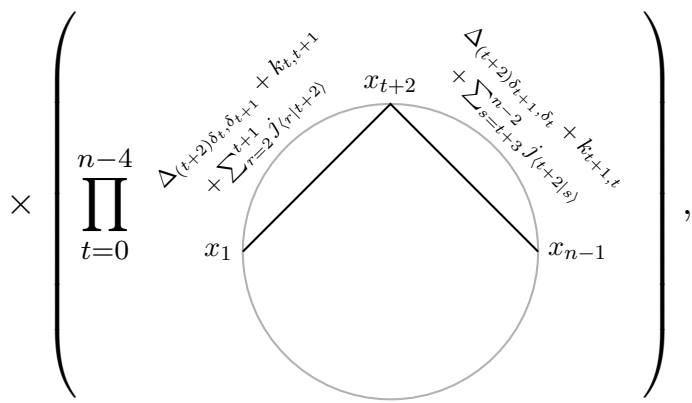

where remember that $\Delta_{\delta_{0}}=\Delta_{1}$ and $k_{0}=k_{n-3}=0$ as well. Comparing with (3.7), we observe that (3.13) has precisely the right position space dependence of the $(n-1)$-point conformal block obtained in the OPE limit $x_{n} \rightarrow x_{n-1}$. Indeed, when $k_{n-3}=0$, it is straightforward to check that the coefficient in (3.7) can be expressed in terms of the coefficient associated with the $(n-1)$-point block times some "extraneous factors" of Euler Gamma function and Pochhammer symbol, as shown:

$$
\begin{aligned}
& \Delta_{1}, \Delta_{\delta_{1}}, \ldots, \Delta_{\delta_{n-3}}, \Delta_{n} ; \Delta_{2}, \ldots, \Delta_{n-1}
\end{aligned}
$$

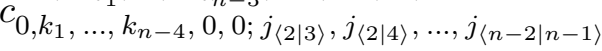

$$
\begin{aligned}
& =c_{0, k_{1}, \ldots, k_{n-4}, 0 ; j_{\langle 2 \mid 3\rangle}, j_{\langle 2 \mid 4\rangle}, \ldots, j_{\langle n-3 \mid n-2\rangle}}^{\Delta_{1}, \Delta_{\delta_{1}}, \ldots, \Delta_{\delta_{n-4}}, \Delta_{\delta_{n-3}} ; \Delta_{2}, \ldots, \Delta_{n-2}} \frac{\Gamma\left(1-\Delta_{\delta_{n-3} n,(n-1)}\right) \Gamma\left(1-\Delta_{1 \delta_{n-3}, 2 \ldots(n-2)}\right)}{\Gamma\left(1-\Delta_{1 n, 2 \ldots(n-1)}\right) \Gamma\left(1-\Delta_{\delta_{n-3}}\right)} \\
& \times\left(\prod_{\ell=2}^{n-2} \frac{(-1)^{j_{\langle\ell \mid n-1\rangle}}}{j_{\langle\ell \mid n-1\rangle} !}\right)\left(\prod_{t=0}^{n-4}\left(\Delta_{(t+2) \delta_{t+1}, \delta_{t}}+k_{t+1, t}+\sum_{s=t+3}^{n-2} j_{\langle t+2 \mid s\rangle}\right)_{j_{\langle t+2 \mid n-1\rangle}}\right) \\
& \times\left(\Delta_{(n-1) \delta_{n-3}, n}\right)_{\sum_{r=2}^{n-2} j_{\langle r \mid n-1\rangle}} \frac{\left(\Delta_{1 n, 2 \ldots(n-1)}\right)_{-\sum_{2 \leq r<s \leq n-1} j_{\langle r \mid s\rangle}}}{\left(\Delta_{1 \delta_{n-3}, 2 \ldots(n-2)}\right)_{-\sum_{2 \leq r<s \leq n-2} j_{\langle r \mid s\rangle}}},
\end{aligned}
$$

where we remember to set $\delta_{0}=\Delta_{1}$ and $k_{0}=k_{n-3}=0$. Thus, we obtain the correct OPE limit (1.8)-(1.9), provided the following $(n-3)$-dimensional coordinate independent sum over the indices $j_{\langle 2 \mid n-1\rangle}, \ldots, j_{\langle n-2 \mid n-1\rangle}$, which involves the "extraneous factors" in (3.14), 
evaluates to unity:

$$
\begin{aligned}
1 \stackrel{!}{=} & \frac{\Gamma\left(1-\Delta_{\delta_{n-3} n,(n-1)}\right) \Gamma\left(1-\Delta_{1 \delta_{n-3}, 2 \ldots(n-2)}\right)}{\Gamma\left(1-\Delta_{1 n, 2 \ldots(n-1)}\right) \Gamma\left(1-\Delta_{\delta_{n-3}}\right)} \\
& \times \sum_{\substack{j_{\langle 2 \mid n-1\rangle}, \ldots, j_{\langle n-2 \mid n-1\rangle}=0}}^{\infty}\left(\prod_{\ell=2}^{n-2} \frac{(-1)^{j_{\langle\ell \mid n-1\rangle}}}{j_{\langle\ell \mid n-1\rangle} !}\right) \\
& \times\left(\prod_{t=0}^{n-4}\left(\Delta_{(t+2) \delta_{t+1}, \delta_{t}}+k_{t+1, t}+\sum_{s=t+3}^{n-2} j_{\langle t+2 \mid s\rangle}\right)_{j_{\langle t+2 \mid n-1\rangle}}\right) \\
& \times\left(\Delta_{(n-1) \delta_{n-3}, n}\right)_{\sum_{r=2}^{n-2} j_{\langle r \mid n-1\rangle}} \frac{\left(\Delta_{1 n, 2 \ldots(n-1)}\right)_{-\sum_{2 \leq r<s \leq n-1} j_{\langle r \mid s\rangle}}}{\left(\Delta_{1 \delta_{n-3}, 2 \ldots(n-2)}\right)_{-\sum_{2 \leq r<s \leq n-2} j_{\langle r \mid s\rangle}}} .
\end{aligned}
$$

Indeed, this turns out to be the case, as shown in appendix C.1.

\subsection{Conformal Casimir check}

Having established the OPE limits for the $n$-point conjecture, we now turn our attention to the multipoint Casimir equations (1.7), repeated below:

$$
\begin{aligned}
\left(\mathcal{L}^{(1)}+\cdots+\mathcal{L}^{(K)}\right)^{2} W_{\Delta_{\delta_{1}} ; \ldots ; \Delta_{\delta_{n-3}}}^{\Delta_{1}, \Delta_{2}, \ldots, \Delta_{n-1}, \Delta_{n}}\left(x_{1}, \ldots, x_{n}\right) \\
\quad=C_{2}\left(\Delta_{\delta_{K-1}}\right) W_{\Delta_{\delta_{1}} ; \ldots ; \Delta_{\delta_{n-3}}}^{\Delta_{1}, \Delta_{2}, \ldots, \Delta_{n-1}, \Delta_{n}}\left(x_{1}, \ldots, x_{n}\right)
\end{aligned}
$$

for all $2 \leq K \leq n-2$, where $\mathcal{L}_{A B}^{(r)}$ are the generators of the Euclidean conformal group acting on and built out of the coordinate $x_{r}, A, B$ are $\mathrm{SO}(d+1,1)$ indices, and $\left(\mathcal{L}^{(r)}\right)^{2}=$ $1 / 2 \mathcal{L}_{A B}^{(r)} \mathcal{L}^{(r) A B}$. In embedding space $\mathbb{R}^{d+1,1}$, these generators act linearly as Lorentz group generators in $d+2$ dimensions,

$$
\mathcal{L}_{A B}^{(r)}=-i\left(X_{A}^{r} \frac{\partial}{\partial X^{r B}}-X_{B}^{r} \frac{\partial}{\partial X^{r A}}\right)
$$

where $X^{r} \in \mathbb{R}^{d+1,1}$ are embedding space coordinates which upon taking an appropriate section give the corresponding Poincaré coordinates $x_{r}$. In the rest of this section, $x_{r}$ will refer to a boundary coordinate so that $X^{r}$ will be a null coordinate in embedding space with $X^{r} \cdot X^{r}=0$ (no sum over $r$ ). Consequently, in embedding space, $x_{r s}^{2}=-2 X^{r} \cdot X^{s}$, and

$$
\begin{aligned}
\mathcal{L}_{A B}^{(r)} \frac{1}{\left(-2 X^{r} \cdot X^{s}\right)^{\Delta}} & =-2 i \Delta \frac{X_{A}^{r} X_{B}^{s}-X_{B}^{r} X_{A}^{s}}{\left(-2 X^{r} \cdot X^{s}\right)^{\Delta+1}} \\
\mathcal{L}^{(r) A B} \mathcal{L}_{A B}^{(r)} \frac{1}{\left(-2 X^{r} \cdot X^{s}\right)^{\Delta}} & =\frac{2 m_{\Delta}^{2}}{\left(-2 X^{r} \cdot X^{s}\right)^{\Delta}} .
\end{aligned}
$$

While we will not explicitly state it every time, all calculations are worked out in embedding space because of the convenience of working with linear differential operators (see e.g. ref. [55] in the context of the five-point block). 
To prove (3.16), first expand the multipoint Casimir operator

$$
\left(\mathcal{L}^{(1)}+\cdots+\mathcal{L}^{(K)}\right)^{2}=\sum_{i=1}^{K}\left(\mathcal{L}^{(i)}\right)^{2}+\sum_{1 \leq r<s \leq K} \mathcal{L}_{A B}^{(r)} \mathcal{L}^{(s) A B}
$$

We will now work out the action of the individual operators in the expansion on the right hand side above, on the putative conformal block. Summing these contributions up, we will show that it reproduces the right hand side of (3.16).

First, the following action of the quadratic Casimir on the conjectural conformal block (3.7) is easily checked:

$$
\left(\mathcal{L}^{(i)}\right)^{2} W_{\Delta_{\delta_{1}} ; \ldots ; \Delta_{\delta_{n-3}}}^{\Delta_{1}, \Delta_{2}, \ldots, \Delta_{n-1}, \Delta_{n}}\left(x_{1}, \ldots, x_{n}\right)=m_{\Delta_{i}}^{2} W_{\Delta_{\delta_{1}} ; \ldots ; \Delta_{\delta_{n-3}}}^{\Delta_{1}, \Delta_{2}, \ldots, \Delta_{n-1}, \Delta_{n}}\left(x_{1}, \ldots, x_{n}\right),
$$

for all $1 \leq i \leq n$ and for all $n$. This follows from the explicit conformal dimension assignment of (3.7) and the following obvious identity

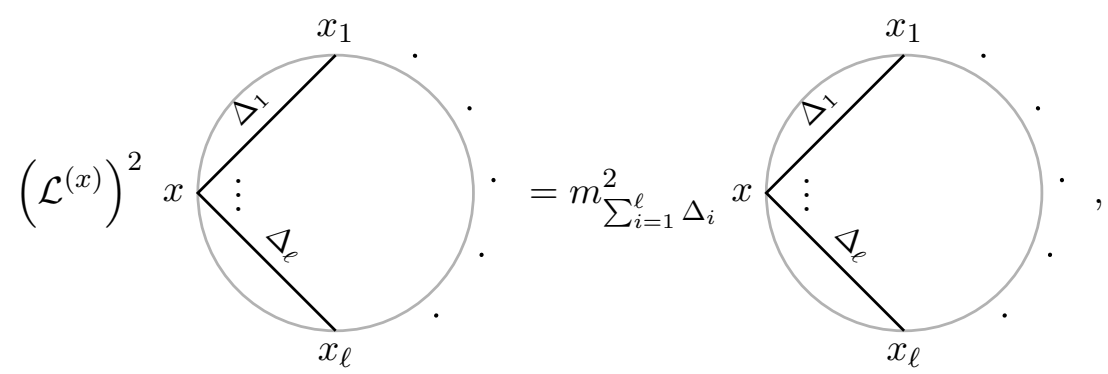

where as explained in figure 2, the solid lines joining together boundary points are to be interpreted as a boundary contractions of the form $\left(x_{i j}^{2}\right)^{-\Delta}$. The action of the cross-term in (3.19) on the putative conformal block (3.7) involves terms of the form

$$
I \equiv \mathcal{L}_{A B}^{(r)} \mathcal{L}^{(s) A B}\left[\Delta_{\langle r \mid s\rangle}\right.
$$

with $1 \leq r<s \leq K$ fixed, and $1 \leq a_{1}<a_{2}<\ldots<a_{n-2} \leq n$ with $a_{1}, a_{2}, \ldots, a_{n-2} \notin\{r, s\}$. Like in the previous subsections, the pictorial representation above is split into a product of Poincaré disks for clarity, but should be understood as merged into a single diagram. We are using the symbols $\langle a \mid b\rangle$ as indices labelling conformal dimensions with $\Delta_{\langle a \mid b\rangle}=\Delta_{\langle b \mid a\rangle}$. Based on convenience, we will sometimes index $a_{i}$ using the subscripts $i=1, \ldots, n-2$, and at other times directly as $a_{i}=1, \ldots, n$ with the restriction that $a_{i} \neq r, s$.

The derivatives acting in (3.22) distribute according to the chain rule to give four terms. We club the two cross-terms together to write $I$ as

$$
I=I_{1}+I_{2}+I_{3}
$$


where we have defined
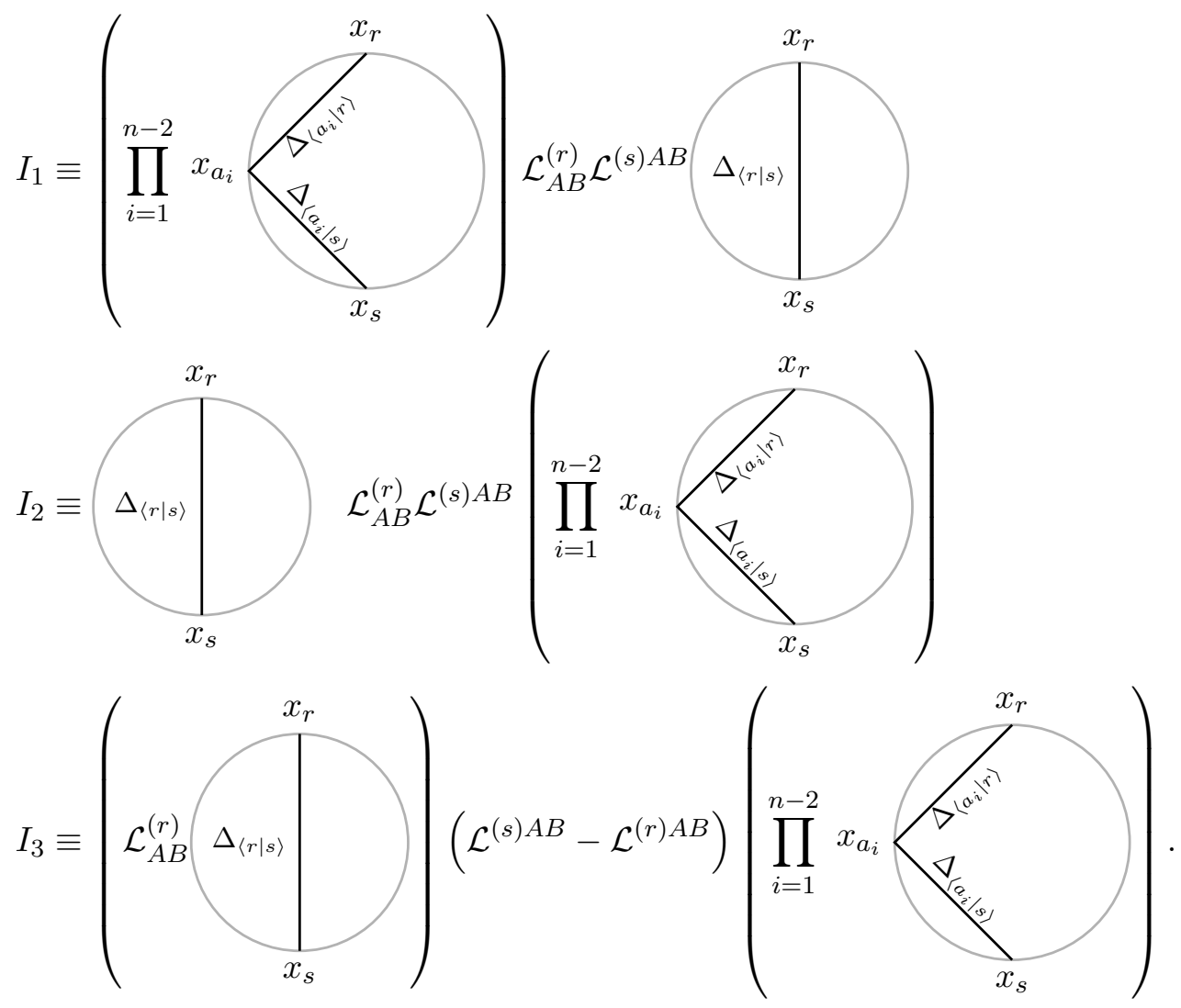

$I_{3}$ repackages the two cross-terms in (3.22) thanks to the obvious identity

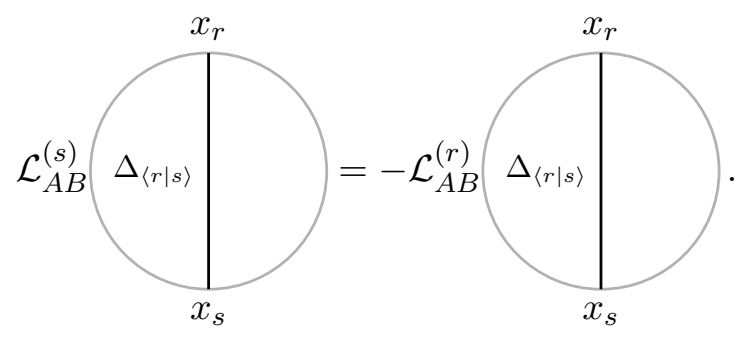

In fact, using this it is straightforward to evaluate $I_{1}$, since

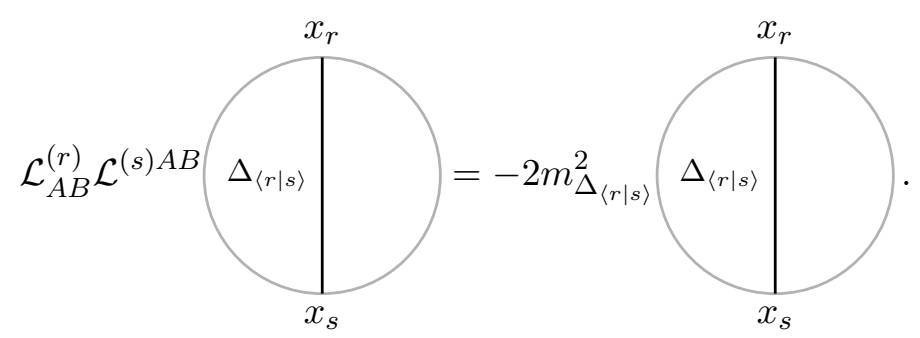


To evaluate $I_{2}$ and $I_{3}$, we use the identity

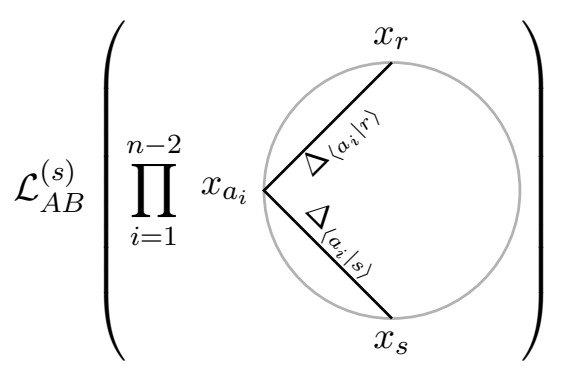

$$
=\left(\sum_{\ell=1}^{n-2} \frac{-i \Delta_{\left\langle a_{\ell} \mid s\right\rangle}\left(2 X_{A}^{s} X_{B}^{a_{\ell}}-2 X_{B}^{s} X_{A}^{a_{\ell}}\right)}{\left(-2 X^{s} \cdot X^{a_{\ell}}\right)}\right)\left(\prod_{i=1}^{n-2} x_{a_{i}}\right.
$$

which is easily checked. Here we have made explicit reference to the embedding space coordinates in the prefactor. ${ }^{12}$ Then, it follows

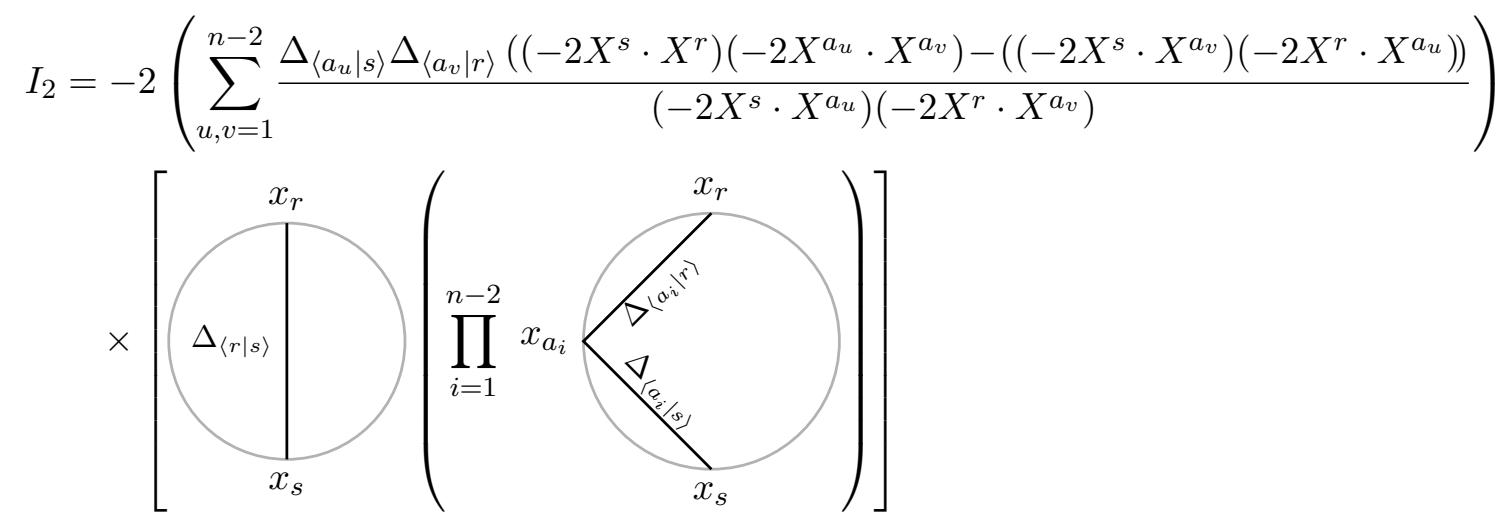

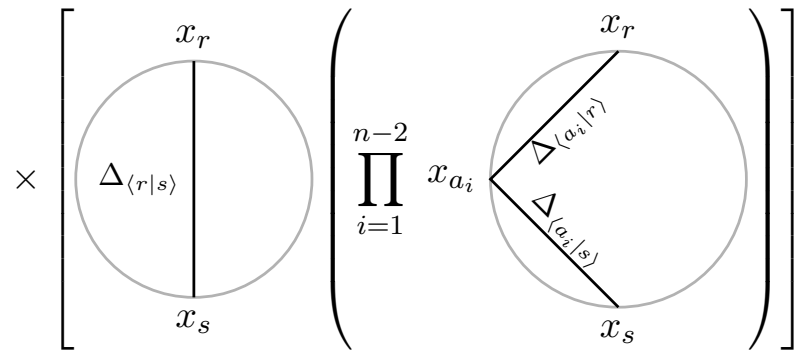

$$
I_{3}=-2 \Delta_{\langle r \mid s\rangle} \sum_{\ell=1}^{n-2}\left(\Delta_{\left\langle a_{\ell} \mid s\right\rangle}+\Delta_{\left\langle a_{\ell} \mid r\right\rangle}\right)
$$

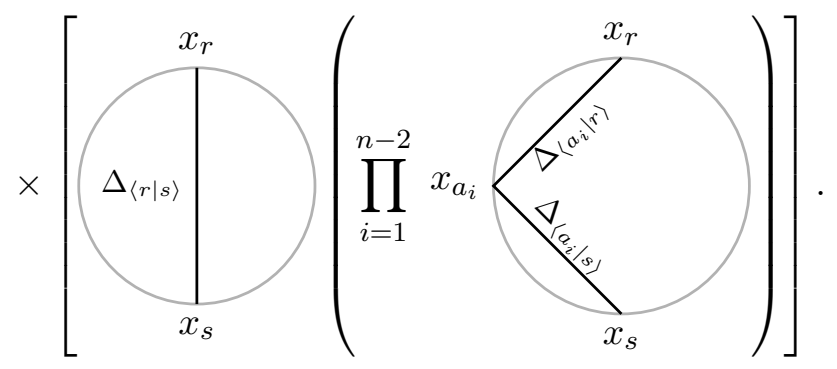

\footnotetext{
${ }^{12}$ At the end of the calculation, we will re-express all expressions in terms of $d$-dimensional boundary coordinates, using the simple identification $\left(-2 X^{i} \cdot X^{j}\right)=x_{i j}^{2}$.
} 
Now rewrite the putative $n$-point conformal block (3.7) as

$$
\begin{aligned}
& W_{\Delta_{\delta_{1}}, \ldots ; \Delta_{\delta_{n-3}}}^{\Delta_{1}, \Delta_{2}, \ldots, \Delta_{n-1}, \Delta_{n}}\left(x_{i}\right)=\sum_{\substack{k_{1}, k_{2}, \ldots, k_{n-3}, j_{\langle 2 \mid 3\rangle}, j_{\langle 2 \mid 4\rangle}, \ldots, j_{\langle n-2 \mid n-1\rangle}=0}}^{\infty} c_{0, k_{1}, k_{2}, \ldots, k_{n-3}, 0 ; j_{\langle 2 \mid 3\rangle}, j_{\langle 2 \mid 4\rangle}, \ldots, j_{\langle n-2 \mid n-1\rangle}}^{\Delta_{1}, \Delta_{\delta_{1}}, \Delta_{\delta_{2}}, \ldots, \Delta_{\delta_{n-1}}, \Delta_{n} ; \Delta_{2}, \ldots, \Delta_{n-1}} \\
& \times \prod_{1 \leq u<v \leq n} \Delta_{\langle u \mid v\rangle}^{x_{u}},
\end{aligned}
$$

where the conformal dimensions $\Delta_{\langle u \mid v\rangle}$, which depend on $k_{1}, \ldots, k_{n-3}$, and various $j_{\langle\cdot \mid \cdot\rangle}$ can be read-off of (3.7). Then, using the computations above, we can evaluate

$$
\begin{aligned}
& \mathcal{L}_{A B}^{(r)} \mathcal{L}^{(s) A B} W_{\Delta_{\delta_{1}} ; \ldots ; \Delta_{\delta_{n-3}}}^{\Delta_{1}, \Delta_{2}, \ldots, \Delta_{n-1}, \Delta_{n}}\left(x_{i}\right) \\
& =-2 \sum_{\substack{k_{1}, k_{2}, \ldots, k_{n-3}, j_{\langle 2 \mid 3\rangle}, j_{\langle 2 \mid 4\rangle}, \ldots, j_{\langle n-2 \mid n-1\rangle}=0}}^{\infty} \begin{array}{c}
\Delta_{1}, \Delta_{\delta_{1}}, \Delta_{\delta_{2}}, \ldots, \Delta_{\delta_{n-3}}, \Delta_{n} ; \Delta_{2}, \ldots, \Delta_{n-1}, \ldots, k_{n-3}, 0 ; j_{\langle 2 \mid 3\rangle}, j_{\langle 2 \mid 4\rangle}, \ldots, j_{\langle n-2 \mid n-1\rangle} \\
c_{0, k_{1}}, k_{2}, k^{\prime}
\end{array} \\
& \times\left(m_{\Delta_{\langle r \mid s\rangle}}^{2}+\Delta_{\langle r \mid s\rangle} \sum_{\ell=1}^{n-2}\left(\Delta_{\left\langle a_{\ell} \mid s\right\rangle}+\Delta_{\left\langle a_{\ell} \mid r\right\rangle}\right)\right. \\
& \left.+\sum_{u, v=1}^{n-2} \Delta_{\left\langle a_{u} \mid s\right\rangle} \Delta_{\left\langle a_{v} \mid r\right\rangle} \frac{\left(x_{r s}^{2} x_{a_{u} a_{v}}^{2}-x_{s a_{v}}^{2} x_{r a_{u}}^{2}\right)}{x_{s a_{u}}^{2} x_{r a_{v}}^{2}}\right) \\
& \times \prod_{1 \leq u<v \leq n} \Delta_{\langle u \mid v\rangle}^{x_{u}},
\end{aligned}
$$

where as before $1 \leq r<s \leq K$ for a fixed $K$ satisfying $2 \leq K \leq n-2$, and $1 \leq a_{1}<a_{2}<$ $\ldots<a_{n-3}<a_{n-2} \leq n$ with $a_{1}, a_{2}, \ldots, a_{n-2} \notin\{r, s\}$.

Combining all the computations (see also footnote 12), the left hand side of (3.16) evaluates to

$$
\begin{aligned}
& \text { l.h.s. }=\sum_{\substack{k_{1}, k_{2}, \ldots, k_{n-3}, j_{\langle 2 \mid 3\rangle}, j_{\langle 2 \mid 4\rangle}, \ldots, j_{\langle n-2 \mid n-1\rangle}=0}}^{\infty} c_{0, k_{1}, \ldots, k_{n-3}, 0 ; j_{\langle 2 \mid 3\rangle}, j_{\langle 2 \mid 4\rangle}, \ldots, j_{\langle n-2 \mid n-1\rangle}}^{\Delta_{1}, \Delta_{\delta_{1}}, \ldots, \Delta_{\delta_{n-3}}, \Delta_{n} ; \Delta_{2}, \ldots, \Delta_{n-1}} \\
& \times\left[\sum_{i=1}^{K} m_{\Delta_{i}}^{2}-2 \sum_{1 \leq r<s \leq K}\left(m_{\Delta_{\langle r \mid s\rangle}^{2}}^{2}+\Delta_{\langle r \mid s\rangle} \sum_{\ell=1}^{n-2}\left(\Delta_{\left\langle a_{\ell} \mid s\right\rangle}+\Delta_{\left\langle a_{\ell} \mid r\right\rangle}\right)\right.\right. \\
& \left.\left.+\sum_{u, v=1}^{n-2} \Delta_{\left\langle a_{u} \mid s\right\rangle} \Delta_{\left\langle a_{v} \mid r\right\rangle} \frac{\left(x_{r s}^{2} x_{a_{u} a_{v}}^{2}-x_{s a_{v}}^{2} x_{r a_{u}}^{2}\right)}{x_{s a_{u}}^{2} x_{r a_{v}}^{2}}\right)\right] \text {, }
\end{aligned}
$$


where we used $\sum_{\substack{u=1 \\ u \neq v}}^{n} \Delta_{\langle u \mid v\rangle}=\Delta_{v}$ for all $1 \leq v \leq n$, which can be easily checked by reading $\Delta_{\langle u \mid v\rangle}$ off of (3.7). Conveniently, a particular partial sum above vanishes, ${ }^{13}$ leaving us with

$$
\begin{aligned}
& \text { 1.h.s. }=\sum_{\substack{k_{1}, k_{2}, \ldots, k_{n-3}, j_{\langle 2 \mid 3\rangle}, j_{\langle 2 \mid 4\rangle}, \ldots, j_{\langle n-2 \mid n-1\rangle}=0}}^{\infty} c_{0, k_{1}, \ldots, k_{n-3}, 0 ; j_{\langle 2 \mid 3\rangle}, j_{\langle 2 \mid 4\rangle}, \ldots, j_{\langle n-2 \mid n-1\rangle}}^{\Delta_{1}, \Delta_{\delta_{1}}, \ldots, \Delta_{\delta_{n-1}, \Delta_{n}, \Delta_{2}, \ldots, \Delta_{n-1}}} \\
& \times\left[\sum_{i=1}^{K} m_{\Delta_{i}}^{2}-2 \sum_{1 \leq r<s \leq K}\left(m_{\Delta_{\langle r \mid s\rangle}}^{2}+\Delta_{\langle r \mid s\rangle} \sum_{\ell=1}^{n-2}\left(\Delta_{\left\langle a_{\ell} \mid s\right\rangle}+\Delta_{\left\langle a_{\ell} \mid r\right\rangle}\right)\right.\right. \\
& \left.\left.-\sum_{\ell=1}^{n-2} \Delta_{\left\langle a_{\ell} \mid s\right\rangle} \Delta_{\left\langle a_{\ell} \mid r\right\rangle}+\sum_{\substack{a_{u}, a_{v}=K+1 \\
a_{u} \neq a_{v}}}^{n} \Delta_{\left\langle a_{u} \mid s\right\rangle} \Delta_{\left\langle a_{v} \mid r\right\rangle} \frac{\left(x_{r s}^{2} x_{a_{u} a_{v}}^{2}-x_{s a_{v}}^{2} x_{r a_{u}}^{2}\right)}{x_{s a_{u}}^{2} x_{r a_{v}}^{2}}\right)\right] .
\end{aligned}
$$

The position space dependence above can be re-expressed in terms of the cross-ratios defined in (1.6) by employing the form (3.10) of the $n$-point block and observing that

$$
\begin{aligned}
& \frac{x_{s r}^{2} x_{a_{u} a_{v}}^{2}}{x_{s a_{u}}^{2} x_{r a_{v}}^{2}}= \begin{cases}u_{s-1} u_{s} \ldots u_{a_{u}-2} \frac{w_{r ; s} w_{a_{u} ; a_{v}}}{w_{s ; a_{u}} w_{r ; a_{v}}} & K+1 \leq a_{u}<a_{v} \leq n-1 \\
u_{s-1} u_{s} \ldots u_{a_{v}-2} \frac{w_{r ; s} w_{a_{v} ; a_{u}}}{w_{s ; a_{u}} w_{r ; a_{v}}} & K+1 \leq a_{v}<a_{u} \leq n-1 \\
u_{s-1} u_{s} \ldots u_{a_{u}-2} \frac{w_{r ; s}}{w_{s ; a_{u}}} & K+1 \leq a_{u} \leq n-1, a_{v}=n \\
u_{s-1} u_{s} \ldots u_{a_{v}-2} \frac{w_{r ; s}}{w_{r ; a_{v}}} & K+1 \leq a_{v} \leq n-1, a_{u}=n\end{cases} \\
& \frac{x_{s a_{v}}^{2} x_{r a_{u}}^{2}}{x_{s a_{u}}^{2} x_{r a_{v}}^{2}}=\frac{w_{s ; a_{v}} w_{r ; a_{u}}}{w_{s ; a_{u}} w_{r ; a_{v}}},
\end{aligned}
$$

for $1 \leq r<s \leq K$, and a fixed $K$ satisfying $2 \leq K \leq n-2$. Substituting this back in the left hand side, we get

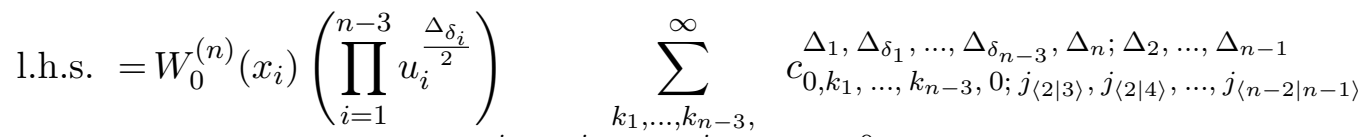

$$
\begin{aligned}
& j_{\langle 2 \mid 3\rangle}, j_{\langle 2 \mid 4\rangle}, \ldots, j_{\langle n-2 \mid n-1\rangle}=0 \\
& \times\left\{\sum_{i=1}^{K} m_{\Delta_{i}}^{2}-2 \sum_{1 \leq r<s \leq K}\left[m_{\Delta_{\langle r \mid s\rangle}^{2}}^{2}+\Delta_{\langle r \mid s\rangle} \sum_{\ell=1}^{n-2}\left(\Delta_{\left\langle r \mid a_{\ell}\right\rangle}+\Delta_{\left\langle s \mid a_{\ell}\right\rangle}\right)-\sum_{\ell=1}^{n-2} \Delta_{\left\langle r \mid a_{\ell}\right\rangle} \Delta_{\left\langle s \mid a_{\ell}\right\rangle}\right.\right.
\end{aligned}
$$

${ }^{13}$ More precisely, the following partial sum vanishes,

$$
\sum_{1 \leq r<s \leq K} \sum_{\substack{a_{u}, a_{v}=1 \\ a_{u}, a_{v} \notin\{r, s\}, a_{u} \neq a_{v}}}^{K} \Delta_{\left\langle a_{u} \mid s\right\rangle} \Delta_{\left\langle a_{v} \mid r\right\rangle} \frac{\left(x_{r s}^{2} x_{a_{u} a_{v}}^{2}-x_{s a_{v}}^{2} x_{r a_{u}}^{2}\right)}{x_{s a_{u}}^{2} x_{r a_{v}}^{2}}=0,
$$

which is proven in appendix C.2. 


$$
\begin{aligned}
& +\sum_{K+1 \leq a_{u}<a_{v} \leq n-1} \Delta_{\left\langle r \mid a_{v}\right\rangle} \Delta_{\left\langle s \mid a_{u}\right\rangle}\left(u_{s-1} u_{s} \ldots u_{a_{u}-2} \frac{w_{r ; s} w_{a_{u} ; a_{v}}}{w_{s ; a_{u}} w_{r ; a_{v}}}-\frac{w_{s ; a_{v}} w_{r ; a_{u}}}{w_{s ; a_{u}} w_{r ; a_{v}}}\right) \\
& +\sum_{K+1 \leq a_{v}<a_{u} \leq n-1} \Delta_{\left\langle r \mid a_{v}\right\rangle} \Delta_{\left\langle s \mid a_{u}\right\rangle}\left(u_{s-1} u_{s} \ldots u_{a_{v}-2} \frac{w_{r ; s} w_{a_{v} ; a_{u}}}{w_{s ; a_{u}} w_{r ; a_{v}}}-\frac{w_{s ; a_{v}} w_{r ; a_{u}}}{w_{s ; a_{u}} w_{r ; a_{v}}}\right) \\
& +\sum_{a_{u}=K+1}^{n-1} \Delta_{\langle r \mid n\rangle} \Delta_{\left\langle s \mid a_{u}\right\rangle}\left(u_{s-1} u_{s} \ldots u_{a_{u}-2} \frac{w_{r ; s}}{w_{s ; a_{u}}}-\frac{w_{r ; a_{u}}}{w_{s ; a_{u}}}\right) \\
& \left.\left.+\sum_{a_{v}=K+1}^{n-1} \Delta_{\left\langle r \mid a_{v}\right\rangle} \Delta_{\langle s \mid n\rangle}\left(u_{s-1} u_{s} \ldots u_{a_{v}-2} \frac{w_{r ; s}}{w_{r ; a_{v}}}-\frac{w_{s ; a_{v}}}{w_{r ; a_{v}}}\right)\right]\right\} \\
& \times\left(\prod_{i=1}^{n-3} u_{i}^{k_{i}}\right)\left(\prod_{2 \leq r<s \leq n-1} w_{r ; s}^{j\langle r \mid s\rangle}\right) .
\end{aligned}
$$

This must be shown to equal the right hand side of (3.16), which can be written as

$$
\begin{aligned}
& \text { r.h.s. }=m_{\Delta_{\delta_{K-1}}}^{2} W_{0}^{(n)}\left(x_{i}\right)\left(\prod_{i=1}^{n-3} u_{i}^{\frac{\Delta_{\delta_{i}}}{2}}\right) \\
& \times \sum_{\substack{k_{1}, \ldots, k_{n-3}, j_{\langle 2 \mid 3\rangle}, j_{\langle 2 \mid 4\rangle}, \ldots, j_{\langle n-2 \mid n-1\rangle}=0}}^{\infty} c_{0, k_{1}, \ldots, k_{n-3}, 0 ; j_{\langle 2 \mid 3\rangle}, j_{\langle 2 \mid 4\rangle}, \ldots, j_{\langle n-2 \mid n-1\rangle}, \Delta_{\delta_{1}}, \ldots, \Delta_{\delta_{n-3}}, \Delta_{n} ; \Delta_{2}, \ldots, \Delta_{n-1}}^{\infty} \\
& \times\left(\prod_{i=1}^{n-3} u_{i}^{k_{i}}\right)\left(\prod_{2 \leq r<s \leq n-1} w_{r ; s}^{j_{\langle r \mid s\rangle}}\right),
\end{aligned}
$$

after substituting $C_{2}\left(\Delta_{\delta_{K-1}}\right)=m_{\Delta_{\delta_{K-1}}}^{2}$.

By integer shifting appropriate integral parameters $k_{i}, j_{\langle\cdot \mid \cdot\rangle}$, we can make the position space dependence of the combination (l.h.s. - r.h.s.) identical across individual terms. For instance, if a term contains an extra factor of $u_{i}$, then shift $k_{i} \rightarrow k_{i}-1$. Similarly, if a term has an extra factor of $w_{r ; s}$, shift $j_{\langle r \mid s\rangle} \rightarrow j_{\langle r \mid s\rangle}-1$. Subsequently, after some further simplifications (see appendix C.3 for calculational details), we end up with

$$
\begin{aligned}
& \text { l.h.s. }- \text { r.h.s. }=W_{0}^{(n)}\left(x_{i}\right)\left(\prod_{i=1}^{n-3} u_{i}^{\frac{\Delta_{\delta_{i}}}{2}}\right) \\
& \times \sum_{\substack{k_{1}, \ldots, k_{n-3}, j_{\langle 2 \mid 3\rangle}, j_{\langle 2 \mid 4\rangle}, \ldots, j_{\langle n-2 \mid n-1\rangle}=0}}^{\infty}\left(4 k_{K-1}\left(\delta_{K-1}+k_{K-1}-d / 2\right) c_{(\cdot)}\right. \\
& \left.-4 \sum_{1 \leq r<s \leq K} \widetilde{c}_{\left(k_{s-1}-1, k_{s}-1, \ldots, k_{a_{u}-2}-1, j_{\langle r \mid s\rangle}-1, j_{\left\langle a_{u} \mid a_{v}\right\rangle}-1, j_{\left\langle s \mid a_{u}\right\rangle}+1, j_{\left\langle r \mid a_{v}\right\rangle}+1\right)}^{r_{k}}\right) \\
& K+1 \leq a_{u}<a_{v} \leq n \\
& \times\left(\prod_{i=1}^{n-3} u_{i}^{k_{i}}\right)\left(\prod_{2 \leq r<s \leq n-1} w_{r ; s}^{j_{\langle r \mid s\rangle}}\right)
\end{aligned}
$$


where we have defined the scaled (tilded) coefficient

$$
\widetilde{c}_{0, k_{1}, \ldots, k_{n-3}, 0 ; j_{\langle 2 \mid 3\rangle}, j_{\langle 2 \mid 4\rangle}, \ldots, j_{\langle n-2 \mid n-1\rangle}, a_{v}}^{r\left(k_{1}\right.} \equiv c_{0, k_{1}, \ldots, k_{n-3}, 0 ; j_{\langle 2 \mid 3\rangle}, j_{\langle 2 \mid 4\rangle}, \ldots, j_{\langle n-2 \mid n-1\rangle}}^{\Delta_{1}, \Delta_{\delta_{1}}, \ldots, \Delta_{\delta_{n-2}}, \Delta_{n} ; \Delta_{2}, \ldots, \Delta_{n-1}} \Delta_{\left\langle r \mid a_{v}\right\rangle} \Delta_{\left\langle s \mid a_{u}\right\rangle},
$$

and for brevity, we are using the short-hands $c_{(\cdot)}, \widetilde{c}_{\left(k_{i} \pm 1, j_{\langle r \mid s\rangle} \pm 1, \ldots\right)}^{r ; a_{u}, a_{v}}$ to stand for

$$
\begin{gathered}
c_{(\cdot)} \equiv c_{0, k_{1}, \ldots, k_{n-3}, 0 ; j_{\langle 2 \mid 3\rangle}, j_{\langle 2 \mid 4\rangle}, \ldots, j_{\langle n-2 \mid n-1\rangle}}^{\Delta_{1}, \Delta_{\delta_{1}}, \ldots, \Delta_{\delta_{n-3}}, \Delta_{n} ; \Delta_{2}, \ldots, \Delta_{n-1}} \\
\left.\widetilde{c}_{\left(k_{i} \pm 1, j_{\langle r \mid s\rangle} \pm 1, \ldots\right)}^{r, s ; a_{u}, a_{v}} \equiv \widetilde{c}_{0, k_{1}, \ldots, k_{n-3}, 0 ; j_{\langle 2 \mid 3\rangle}, j_{\langle 2 \mid 4\rangle}, \ldots, j_{\langle n-2 \mid n-1\rangle}}^{r, s}\right|_{k_{i} \rightarrow k_{i} \pm 1, j_{\langle r \mid s\rangle} \rightarrow j_{\langle r \mid s\rangle} \pm 1, \ldots},
\end{gathered}
$$

that is, coefficients with (un)shifted integral parameters.

Thus to show that the conformal Casimir equations are satisfied, we need to show (3.37) vanishes identically. Since it must vanish irrespective of the choice of boundary coordinates, each individual term in the sum must vanish. That is, we need to show

$$
\begin{aligned}
& 4 k_{K-1}\left(\delta_{K-1}+k_{K-1}-d / 2\right) c_{(\cdot)} \\
& -4 \sum_{1 \leq r<s \leq K} \widetilde{c}_{\left(k_{s-1}-1, k_{s}-1, \ldots, k_{a_{u}-2}-1, j_{\langle r \mid s\rangle}-1, j_{\left\langle a_{u} \mid a_{v}\right\rangle}-1, j_{\left\langle s \mid a_{u}\right\rangle}+1, j_{\left\langle r \mid a_{v}\right\rangle}+1\right)} \stackrel{!}{=} 0 \\
& K+1 \leq a_{u}<a_{v} \leq n
\end{aligned}
$$

for all $2 \leq K \leq n-2$, and for all $k_{1}, \ldots, k_{n-3}, j_{\langle 2 \mid 3\rangle}, j_{\langle 2 \mid 4\rangle}, \ldots, j_{\langle n-2 \mid n-1\rangle} \in \mathbb{Z}^{\geq 0}{ }^{14}$ We proved this analytically for all $n \leq 7$ (and all admissible values of $K$ ). The calculations are straightforward, though lengthy and are provided in the supplementary material as a Mathematica notebook with the submission. The key tool useful for proving (3.40) is an $(n-4)$-fold application of the hypergeometric identity $(2.11)$, once for each factor of ${ }_{3} F_{2}$ in the original expansion coefficient (3.8). For higher $n$, the analytics become particularly lengthy and unwieldy, but we cannot rule out a simple proof may exist. Nevertheless, it is straightforward to check (3.40) numerically for arbitrary values of $n$ and $K$ to arbitrary numerical precision; such a check is also included in the same Mathematica notebook. Thus while (3.40) remains to be established fully analytically for $n \geq 8$, in our view the highly non-trivial numerical checks provide convincing supporting evidence.

\section{Discussion}

In this paper, we used the holographic principle, particularly its theory-independent kinematic aspects, to obtain for the first time explicit expressions for a class of multipoint conformal blocks. We started by establishing the holographic geodesic diagram representations of $d$-dimensional comb channel six-point $((2.6)-(2.9))$ and seven-point ((B.1)-(B.4)) scalar conformal blocks involving scalar exchanges. From them we recovered power series expansions for respectively the four- and five-point blocks via a double-OPE limit. The explicit low-point examples, along with the holographic dual of the five-point block [55],

\footnotetext{
${ }^{14}$ Due to the symmetry of the conjectural conformal block, we need only show (3.40) for $2 \leq K \leq\lfloor n / 2\rfloor$ where $\lfloor\cdot\rfloor$ is the floor function; the rest follow after a simple relabelling.
} 
allowed a generalization to the holographic dual of the $(n+2)$-point block for arbitrary $n$ ((3.1)-(3.4)). Like the low-point examples, it was expressible in terms of a linear combination of $(n+2)$-point geodesic diagrams involving two bulk geodesic integrals. Its double-OPE limit then led us to a power series expansion for a scalar $n$-point block in (3.7) (given also in (1.1)-(1.4)), which is the main technical result of this paper and proven in sections 3.2-3.3. Obtaining the holographic representations of low-point but non-trivial comb channel blocks served a crucial purpose in this paper. The low-point examples were simple enough that we needed only existing technology [56] to obtain them, but non-trivial enough for us to recognize the pattern and guess the form of the holographic dual of an arbitrary-point block.

There are various avenues for further investigation. In this paper, we restricted ourselves to studying $n$-point comb channel scalar conformal blocks involving solely scalar exchanges. To be able to set up an alternative $n$-point conformal bootstrap involving scalar $n$-point functions for all $n$, one must also have in hand higher-point scalar blocks involving exchange of other non-trivial representations of the Lorentz group. It should be possible to generate $n$-point comb channel blocks involving both external and internal spin exchanges by, for example, operating on the blocks obtained in this paper via weight-shifting operators [27]. It should also be possible to generate higher-point spinning geodesic diagram representations using the AdS differential operators [34, 35]. Various recursive techniques, when supplemented with the results of this paper, may also turn out to be fruitful.

For setting up the $n$-point conformal bootstrap, one also needs $n$-point blocks in channels other than the comb channel. The number of topologically distinct channels, not related via conformal transformation or simple relabeling, grows quickly with $n$. Thus it is likely inefficient to compute multipoint conformal blocks one specific channel at a time. On the other hand, it is conceivable there exist some version of "Feynman-like" rules for writing out conformal blocks, akin to Feynman rules for Mellin amplitudes [68-70], which can be worked out once and for all. We hope the explicit expressions for the $n$-point comb channel block we obtained in this paper will help elucidate these Feynman-like rules.

For instance, consider the diagrammatic representation of the comb channel conformal block in figure 1. It has two internal cubic vertices where exactly two external legs and one internal leg are incident, and $(n-4)$ internal cubic vertices at which exactly one external leg and two internal legs are incident. In this paper we saw that both the holographic representation as well as power series expansion of the $n$-point comb channel block have precisely $(n-4)$ factors of the hypergeometric ${ }_{3} F_{2}$ function in the explicit expansion. ${ }^{15}$ This is not a coincidence, and is in fact reminiscent of Feynman rules for scalar Mellin amplitudes. Our work suggests a holographic origin for this. Given a conformal block, as argued previously $[55,56]$, a general strategy for extracting its explicit holographic dual

\footnotetext{
${ }^{15}$ One might be tempted to assert from (1.1) that there are in fact $(n-2)$ factors of the hypergeometric function, but the precise count is $(n-4)$ since $k_{0}$ and $k_{n-2}$ vanish by definition (1.4). This is a simple generalization of the previously known cases for scalar blocks with scalar exchanges - the four-point block doesn't have any ${ }_{3} F_{2}$ functions in its double power series expansion [12], while the power series expansion for the $d$-dimensional five-point block obtained in ref. [53] (as well as its holographic representation [55]) carries precisely one factor of the hypergeometric ${ }_{3} F_{2}$ function.
} 
(and consequently a power series expansion via a double-OPE limit as discussed in this paper) is to start with a canonical tree-level AdS diagram. ${ }^{16}$ The AdS diagram should have solely cubic couplings and its direct-channel conformal block decomposition should include the given block. Performing the bulk integrals in the diagram carefully using various two- and three-propagator AdS identities helps extract an explicit representation for the block $[43,55,56]$. Indeed for comb channel blocks, the $(n-4)$ factors of ${ }_{3} F_{2}$ arise due to the presence of $(n-4)$ cubic bulk integrals involving exactly one bulk-to-boundary propagator and two bulk-to-bulk propagators. This integral was fully worked out in ref. [56] and it involves precisely the right factor of the hypergeometric function ${ }_{3} F_{2}$ with precisely the right arguments. ${ }^{17}$ In fact, this general argument should extend to any arbitrary-point scalar conformal block with scalar exchanges in an arbitrary channel. All the necessary three-propagator integrals appearing in such a derivation were worked out in ref. [56]. This should make it tractable to work out the putative Feynman rules for all scalar blocks.

It is interesting to compare our results with the parallel, albeit considerably simpler story in the framework of $p$-adic AdS/CFT, where the conformal group is $\operatorname{PGL}\left(2, \mathbb{Q}_{p^{d}}\right)[71$, 72] ${ }^{18}$ Due to the lack of descendant operators in $p$-adic CFTs [74], the conformal blocks are simply scaling blocks. Nevertheless, analogous to the real conformal blocks, the $p$ adic blocks admit holographic duals, written as geodesic bulk diagrams on the Bruhat-Tits tree $[48,55,56]$. In fact, all results presented in this paper also admit a $p$-adic counterpart - various recent accounts of comparison and translation between objects in the usual (real) and $p$-adic holographic settings can be found in refs. [48, 55, 56, 71, 72, 75-92]. Essentially, to recover the $p$-adic result, one can truncate all power series expansions featured in this paper to their respective first terms, since the infinite multi-fold series expansions in real CFTs sum up descendant contributions which do not exist in $p$-adic CFTs. ${ }^{19}$ Conversely, it is practical to work out holographic duals of blocks in the simpler $p$-adic setting first. This is because the conformal dimension dependence of propagators appearing in the associated $p$-adic geodesic diagrams is identical to that for geodesic diagrams in real CFTs (more precisely, the "primary contribution" is identical). So the simpler $p$-adic technology can be used to figure out in advance the expected primary contribution to conformal blocks in a real CFT; the full block, which sums up also the descendant contributions, can then in principle be determined from conformal invariance.

Finally, a potential practical concern about the power series expansion (1.1) could be that it is not rapidly convergent for operator insertions in the "OPE regime" of the comb channel, i.e. for cross-ratios $u_{i} \ll 1$ and $w_{r ; s} \approx 1$ defined in (1.6) (for all allowed values of the subscripts). However, one can remedy this slow convergence by a simple transformation which re-expresses the series expansion in powers of $w_{r ; s}$ as an expansion

\footnotetext{
${ }^{16}$ The canonical choice for the AdS diagram for extracting the six-point comb channel block was provided in (2.4).

${ }^{17}$ The remaining two bulk integrals involving integration over a product of precisely two bulk-to-boundary propagators and one bulk-to-bulk propagator contribute simply factors of Gamma functions or Pochhammer symbols [56].

${ }^{18}$ Here $\mathbb{Q}_{p^{d}}$ is the unique unramified degree $d$ field extension of the $p$-adic numbers $\mathbb{Q}_{p}$ (see e.g. the book [73]).

${ }^{19}$ For example, the holographic dual of the comb channel $p$-adic block is given by (3.1)-(3.2) except with all integral parameters being summed over set to zero.
} 
in powers of $\left(1-w_{r ; s}\right)$. This is straightforward to work out, though increasingly tedious as $n$ grows. More precisely, to transform (1.1) to a more efficient and rapidly convergent power series expansion for any fixed $n$, the following identity is useful (see e.g. footnote 8 ):

$$
\begin{aligned}
\sum_{j=0}^{\infty} \frac{(-w)^{j}}{j !} & \left(a_{1}\right)_{b_{1}+j}\left(a_{2}\right)_{b_{2}+j}\left(a_{3}\right)_{b_{3}-j} \\
= & \sum_{j=0}^{\infty} \frac{(-1)^{b_{3}} w^{j}}{j !} \frac{\left(a_{1}\right)_{b_{1}+j}\left(a_{2}\right)_{b_{2}+j}}{\left(1-a_{3}\right)_{-b_{3}+j}} \\
= & \frac{(-1)^{b_{1}+b_{2}} \Gamma\left(1-a_{3}\right) \Gamma\left(1-\left(a_{1}+a_{2}+a_{3}\right)\right)}{\Gamma\left(1-\left(a_{1}+a_{3}+b_{1}+b_{3}\right)\right) \Gamma\left(1-\left(a_{2}+a_{3}+b_{2}+b_{3}\right)\right)} \\
& \times \sum_{j=0}^{\infty} \frac{(1-w)^{j}}{j !} \frac{\left(a_{1}\right)_{b_{1}+j}\left(a_{2}\right)_{b_{2}+j}}{\left(a_{1}+a_{2}+a_{3}\right)_{b_{1}+b_{2}+b_{3}+j}}
\end{aligned}
$$

for integers $b_{1}, b_{2}, b_{3}$ and the parameter space where the power series in $w$ and $(1-w)$ are simultaneously convergent; we analytically continue to extend the result outside this convergent regime.

For the four-, five-, six-, and seven-point blocks, we can explicitly check that a repeated application of this identity leads to the following alternate representation (for $n=4,5,6,7$ )

$$
\begin{aligned}
& W_{n}=W_{0}^{(n)}\left(x_{i}\right)\left(\prod_{i=1}^{n-3} u_{i}^{\frac{\Delta_{i}}{2}}\right) \\
& \times \sum_{\substack{k_{1}, \ldots, k_{n-3}, j_{\langle 2 \mid 3\rangle}, j_{\langle 2 \mid 4\rangle}, \ldots, j_{\langle n-2 \mid n-1\rangle}=0}}^{\infty}\left[\left(\prod_{i=1}^{n-3} \frac{u_{i}^{k_{i}}}{k_{i} !}\right)\left(\prod_{2 \leq r<s \leq n-1} \frac{\left(1-w_{r ; s}\right)^{j_{\langle r \mid s\rangle}}}{j_{\langle r \mid s\rangle} !}\right)\right. \\
& \times\left(\prod_{t=0}^{n-3} \frac{\left(1-\Delta_{(t+1) \delta_{t-1}, \delta_{t}}\right)_{k_{t}}\left(1-\Delta_{(t+2) \delta_{t+1}, \delta_{t}}\right)_{k_{t}}}{\left(\Delta_{\delta_{t}}-d / 2+1\right)_{k_{t}}\left(\Delta_{\delta_{t}}\right)_{2 k_{t}+\sum_{2 \leq r<t+2 \leq s \leq n-1} j_{\langle r \mid s\rangle}}}\right. \\
& \times\left(\Delta_{(t+2) \delta_{t}, \delta_{t+1}}\right)_{k_{t, t+1}+\sum_{2 \leq r<t+2} j_{\langle r \mid t+2\rangle}}\left(\Delta_{(t+2) \delta_{t+1}, \delta_{t}}\right)_{k_{t+1, t}+\sum_{t+2<s \leq n-1} j_{\langle t+2 \mid s\rangle}} \\
& \times\left(\Delta_{\delta_{t} \delta_{t+1},(t+2)}\right)_{k_{t(t+1)},+\sum_{2 \leq r<t+2<s \leq n-1} j_{\langle r \mid s\rangle}} \\
& \left.\left.\times{ }_{3} F_{2}\left[\left\{-k_{t},-k_{t+1}, \Delta_{\delta_{t} \delta_{t+1}(t+2)},-\frac{d}{2}\right\} ;\left\{\Delta_{(t+2) \delta_{t+1}, \delta_{t}}-k_{t}, \Delta_{(t+2) \delta_{t}, \delta_{t+1}}-k_{t+1}\right\} ; 1\right]\right)\right] .
\end{aligned}
$$

Recently, a similar power series expansion was obtained for the same five-point block in general spacetime dimensions [53]. There, a different set of conformal cross-ratios were used. We expect it to be possible to establish analytically the equality between the result of ref. [53] and (4.2) for $n=5$, but we have not found any simple transformations which achieve this. However, we have verified numerically that the power series expansion (4.2) matches the one in ref. [53] to desired numerical precision in the mutual regime of convergence of the two series expansions, as expected. While we have not worked out the alternate representation for general $n$, we conjecture this to also hold for all $n \geq 8$. 


\section{Acknowledgments}

I thank Robert Clemenson for valuable discussions and collaboration in the early stages, and Christian B. Jepsen for useful discussions. I would also like to thank Steve Gubser, my Ph.D. advisor. Our joint work from about two years ago [48] served as an important stepping stone and a springboard for many subsequent ideas, including the present work. Steve was an inspiring mentor, an extraordinary physicist and an exceptional human being. I am deeply grateful to Steve Gubser for everything, and would like to dedicate this paper to his fond memory.

\section{A Obtaining the six-point holographic dual}

In this appendix we present the main computational steps for "deriving" the holographic dual of the six-point conformal block described schematically in section 2.1 below equation (2.5); see also refs. [55, 56] where the same systematic procedure is explained and several examples worked out in detail. Strictly speaking this is not a true derivation, but a heuristic method to obtain a reasonable candidate for the holographic dual of the conformal block, which can then be checked against the conformal Casimir equations [55].

As described near (2.5), the following bulk diagram

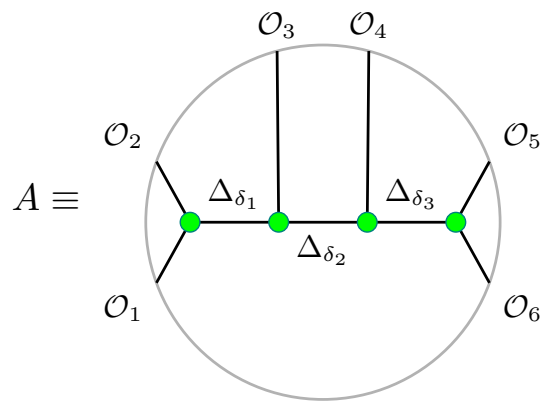

admits a conformal block decomposition in the direct channel such that the term with pure single-trace exchanges is precisely the conformal block we are after, up to a product of four OPE coefficients. In this section we describe how to extract this term. We first recast $A$ into a form involving two geodesic integrals with a double-application of a two-propagator identity which replaces a product of two bulk-to-boundary propagators with a geodesic integral [43], $\hat{K}_{\Delta_{1}} \hat{K}_{\Delta_{2}} \propto \sum_{M} \int_{\gamma} \hat{K}_{\Delta_{1}} \hat{K}_{\Delta_{2}} \hat{G}_{\Delta_{1}+\Delta_{2}+2 M}$ where $\hat{G}_{\Delta}, \hat{K}_{\Delta}$ are respectively the unnormalized bulk-to-bulk and bulk-to-boundary propagators (see figure 2 for more details on propagators). ${ }^{20}$ Using this, we get

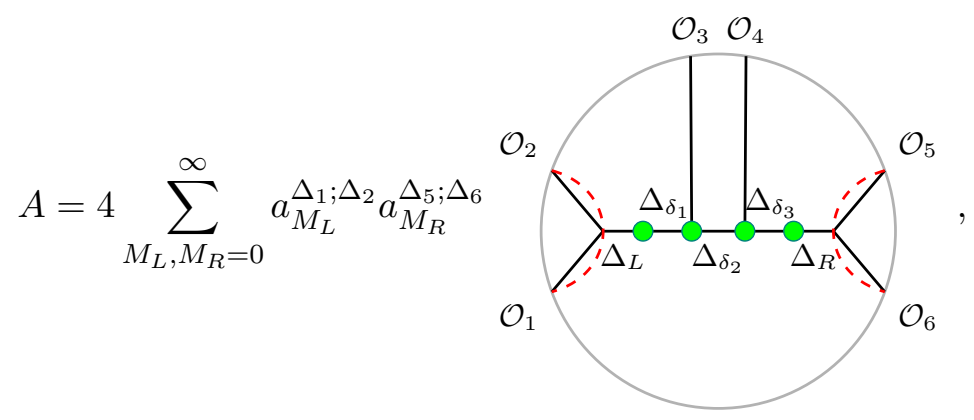

\footnotetext{
${ }^{20}$ Ref. [56, section 3.1] gives a succinct one-page review of all two-propagator identities we will employ in this section.
} 
where

$$
\Delta_{L} \equiv \Delta_{1}+\Delta_{2}+2 M_{L} \quad \Delta_{R} \equiv \Delta_{5}+\Delta_{6}+2 M_{R}
$$

and

$$
a_{M}^{\Delta_{1} ; \Delta_{2}} \equiv \frac{1}{B\left(\Delta_{1}+M, \Delta_{2}+M\right)} \frac{(-1)^{M}}{M !} \frac{\left(\Delta_{1}\right)_{M}\left(\Delta_{2}\right)_{M}}{\left(\Delta_{1}+\Delta_{2}+M-\frac{d}{2}\right)_{M}},
$$

where $B(s, t)$ is the usual Euler Beta function. The diagrammatic notation in (A.2) is explained in figure 2. (Note that we did not differentiate between propagators using the color-codes of figure 2 - it should be obvious which ones are bulk-to-bulk propagators and which ones are bulk-to-boundary propagators in (A.2).) We can now easily evaluate all "two-point" bulk integrals over a product of two bulk-to-bulk propagators. For this we utilize a two-propagator identity which replaces bulk integrals with a linear combination of unintegrated bulk-to-bulk propagators [43] (see also ref. [56, section 3.1]), $\int_{\operatorname{AdS}} \hat{G}_{\Delta_{a}} \hat{G}_{\Delta_{b}} \propto$ $\left(\hat{G}_{\Delta_{a}}-\hat{G}_{\Delta_{b}}\right)$, which leads to

$$
\begin{aligned}
A=4 \sum_{M_{L}, M_{R}=0}^{\infty} \frac{a_{M_{L}}^{\Delta_{1} ; \Delta_{2}} a_{M_{R}}^{\Delta_{5} ; \Delta_{6}}}{\left(m_{\Delta_{L}}^{2}-m_{\Delta_{\delta_{1}}}^{2}\right)\left(-N_{\Delta_{L}}\right)\left(m_{\Delta_{\delta_{3}}}^{2}-m_{\Delta_{R}}^{2}\right) N_{\Delta_{R}}} \\
\quad+(3 \text { more terms }),
\end{aligned}
$$

where $N_{\Delta} \equiv \frac{-\pi^{-d / 2} \Gamma(\Delta)}{(2 \Delta-d) \Gamma(\Delta-d / 2)}$. The four terms in (A.5) (three of which have been suppressed) originate from replacing the two $\int_{\text {AdS }} \hat{G} \hat{G}$ bulk integrals over a product of bulk-to-bulk propagators with a linear combination of bulk-to-bulk propagators $\propto(\hat{G}-\hat{G})$ of scalar fields of appropriate masses. The three suppressed terms correspond to the exchange of double-trace operators in the dual CFT picture and thus play no role in our calculation since we are interested in extracting the conformal block corresponding to the exchange of single-trace operators $[55,56]$. From here on, we will drop these terms.

The $M_{L}, M_{R}$ sums in the first term of (A.5) can be evaluated analytically [56, eq. (4.12)] to give

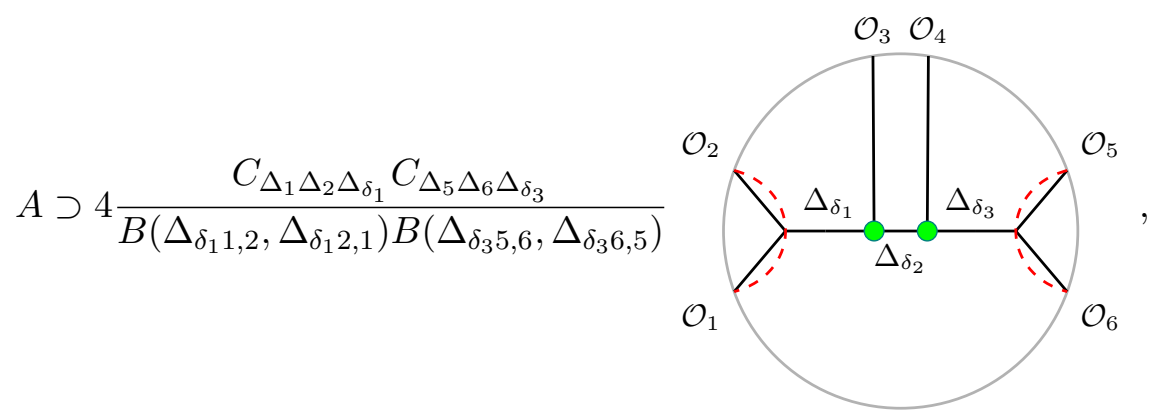

where we are using the symbol $\supset$ to remind the reader we have dropped the three terms in (A.5). 
Next, we employ the split representation [93] on the bulk-to-bulk propagator labelled with the conformal dimension $\Delta_{\delta_{2}}$,

$$
\hat{G}_{\Delta_{\delta_{2}}}\left(z_{1}, z_{2}\right)=\int_{y \in \partial \mathrm{AdS}} \int_{-i \infty}^{i \infty} \frac{d \nu}{2 \pi i} \rho_{\Delta_{\delta_{2}}}(\nu) \hat{K}_{\frac{d}{2}+\nu}\left(y, z_{1}\right) \hat{K}_{\frac{d}{2}-\nu}\left(y, z_{2}\right)
$$

where

$$
\rho_{\Delta} \equiv \frac{-\pi^{-d}}{N_{\Delta}} \frac{1}{m_{\Delta}^{2}-m_{\frac{d}{2}+\nu}^{2}} \frac{\Gamma\left(\frac{d}{2}+\nu\right) \Gamma\left(\frac{d}{2}-\nu\right)}{4 \Gamma(\nu) \Gamma(-\nu)},
$$

with an appropriate choice of the contour of integration [93]. Thus pictorially, we may write (A.6) as

$$
A \supset 4 \frac{C_{\Delta_{1} \Delta_{2} \Delta_{\delta_{1}}} C_{\Delta_{5} \Delta_{6} \Delta_{\delta_{3}}}}{B\left(\Delta_{\delta_{1} 1,2}, \Delta_{\delta_{1} 2,1}\right) B\left(\Delta_{\delta_{3} 5,6}, \Delta_{\delta_{3} 6,5}\right)}
$$

At this point we recognize the remaining two full AdS integrals as three-propagator integrals of the type $\int_{\text {AdS }} \hat{K} \hat{K} \hat{G}$ which can be evaluated explicitly [56, section 3.2.1]. Upon evaluation, such three-propagator integrals can be expressed as products over unintegrated propagators. Like in the case of the $\int_{\mathrm{AdS}} \hat{G} \hat{G}$ identity, the precise form is given as a linear combination of two terms, so that the double-integral in (A.9) leads to four terms. However once again, we are only interested in one of these four terms, the one which comes with the right OPE coefficients as overall factors [55]. We will justify this choice shortly. Focusing on the right term (i.e. dropping the other three), we obtain

$$
A \supset 4 \frac{C_{\Delta_{1} \Delta_{2} \Delta_{\delta_{1}}} C_{\Delta_{5} \Delta_{6} \Delta_{\delta_{3}}}}{B\left(\Delta_{\delta_{1} 1,2}, \Delta_{\delta_{1} 2,1}\right) B\left(\Delta_{\delta_{3} 5,6}, \Delta_{\delta_{3} 6,5}\right)} \int_{-i \infty}^{i \infty} \frac{d \nu}{2 \pi i} \rho_{\Delta_{\delta_{2}}}(\nu) C_{\Delta_{\delta_{1}} \Delta_{3}\left(\frac{d}{2}+\nu\right)} C_{\Delta_{\delta_{3}} \Delta_{4}\left(\frac{d}{2}-\nu\right)}
$$

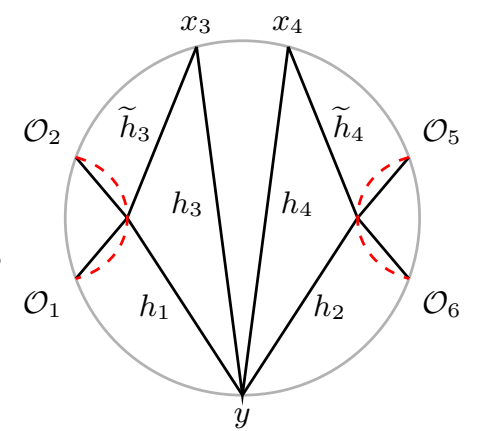


where

$$
c_{k}^{\Delta_{1} ; \Delta_{a} ; \Delta_{2}} \equiv \frac{1}{k !} \frac{\left(\Delta_{a 1,2}\right)_{k}\left(\Delta_{a 2,1}\right)_{k}}{\left(\Delta_{a}-\frac{d}{2}+1\right)_{k}},
$$

and we have defined the dimensions

$$
\begin{array}{ll}
h_{1} \equiv \frac{\Delta_{\delta_{1}}+\frac{d}{2}+\nu-\Delta_{3}}{2}+k_{1} & h_{2} \equiv \frac{\Delta_{\delta_{3}}+\frac{d}{2}-\nu-\Delta_{4}}{2}+k_{3} \\
h_{3} \equiv \frac{\Delta_{3}+\frac{d}{2}+\nu-\Delta_{\delta_{1}}}{2}-k_{1} & h_{4} \equiv \frac{\Delta_{4}+\frac{d}{2}-\nu-\Delta_{\delta_{3}}}{2}-k_{3} \\
\widetilde{h}_{3} \equiv \frac{\Delta_{\delta_{1}}+\Delta_{3}-\frac{d}{2}-\nu}{2}+k_{1} & \widetilde{h}_{4} \equiv \frac{\Delta_{\delta_{3}}+\Delta_{4}-\frac{d}{2}+\nu}{2}+k_{3} .
\end{array}
$$

We would now like to justify why we chose to focus on this term. Note that the $\nu$-dependent integrand in (A.10) has simple poles at $\nu= \pm\left(\frac{d}{2}-\Delta_{\delta_{2}}\right)$. The residue at either of these leads immediately to an overall factor of OPE coefficients exactly as prescribed in (2.5). Thus for the direct-channel conformal block, we need only focus on the term in (A.10), and in fact only on the residue at either of the two poles $\nu= \pm\left(\frac{d}{2}-\Delta_{\delta_{2}}\right)$. The choice of the correct pole is dictated by the position-dependent expression obtained after doing the $y$-boundary integral in (A.10), to which we now turn.

The boundary integral in (A.10) is a variant of the usual four-point conformal integral, which in embedding space takes the form

$$
J \equiv \int_{Y^{2}=0} d Y \frac{1}{\left(-2 X_{3} \cdot Y\right)^{h_{3}}} \frac{1}{\left(-2 X_{4} \cdot Y\right)^{h_{4}}} \frac{1}{\left(-2 W_{1} \cdot Y\right)^{h_{1}}} \frac{1}{\left(-2 W_{2} \cdot Y\right)^{h_{2}}}
$$

with $h_{1}+h_{2}+h_{3}+h_{4}=d$, where the embedding space coordinates $Y, X_{3}, X_{4} \in \mathbb{R}^{d+1,1}$ are restricted to the null cone $Y \cdot Y=Y^{2}=X_{3}^{2}=X_{4}^{2}=0$ whereas $W_{1}, W_{2} \in \mathbb{R}^{d+1,1}$ lie on the hyperboloid $W_{1}^{2}=W_{2}^{2}=-1$. This integral can be worked out by applying Schwinger parametrization and subsequently turning it into a Mellin-Barnes integral. Since these techniques are not new ${ }^{21}$ in the interest of keeping the appendix to a reasonable length, we refrain from presenting all the standard intermediate steps and refer the reader to the references in footnote 21. These computations lead to the following combination of Mellin-Barnes integrals

$$
\begin{aligned}
J= & \frac{\pi^{d / 2}}{\prod_{i=1}^{4} \Gamma\left(h_{i}\right)} \int_{\epsilon-i \infty}^{\epsilon+i \infty} \frac{d s d t}{(2 \pi i)^{2}} \frac{\Gamma(-s) \Gamma(-t)}{\left(-2 W_{1} \cdot X_{4}\right)^{-s}\left(-2 W_{2} \cdot X_{4}\right)^{-t}} \frac{\Gamma\left(h_{4}+s+t\right)}{\left(-2 X_{3} \cdot X_{4}\right)^{h_{4}+s+t}} \\
& \times\left[\sum_{m_{1}, m_{2}=0}^{\infty} \frac{(-1)^{m_{1}+m_{2}}}{m_{1} ! m_{2} !} \frac{\Gamma\left(-s+h_{23,14}-m_{1}+m_{2}\right) \Gamma\left(-t+h_{13,24}+m_{1}-m_{2}\right)}{\left(-2 W_{2} \cdot X_{3}\right)^{-s+h_{23,14}-m_{1}+m_{2}}\left(-2 W_{1} \cdot X_{3}\right)^{-t+h_{13,24}+m_{1}-m_{2}}}\right. \\
& \times \frac{\Gamma\left(s+t+h_{124,3}+m_{1}+m_{2}\right)}{\left(-2 W_{1} \cdot W_{2}\right)^{s+t+h_{124,3}+m_{1}+m_{2}}}
\end{aligned}
$$

\footnotetext{
${ }^{21}$ For example, the same techniques are detailed in ref. [56, appendix B.1] for three-point variants of the four-point integral (A.13) (obtained by, for example, setting one of $h_{1}, h_{2}, h_{3}$ or $h_{4}$ to zero while maintaining the sum to be $d$ ). A related four-point conformal integral (for $X_{3}, X_{4}, W_{1}, W_{2}$ in (A.13) all on the null cone) has also been previously worked out using the same methods [53, appendix B.1].
} 


$$
\begin{aligned}
& +\left(\sum_{m_{1}, m_{2}=0}^{\infty} \frac{(-1)^{m_{1}+m_{2}}}{m_{1} ! m_{2} !} \frac{\Gamma\left(-s-t+h_{3,4}+m_{2}\right) \Gamma\left(s-h_{23,14}-m_{1}-m_{2}\right)}{\left(-2 W_{1} \cdot X_{3}\right)^{-s-t+h_{3,4}+m_{1}}\left(-2 W_{2} \cdot X_{3}\right)^{-m_{1}}}\right. \\
& \left.\left.\quad \times \frac{\Gamma\left(t+h_{2}+m_{1}+2 m_{2}\right)}{\left(-2 W_{1} \cdot W_{2}\right)^{t+h_{2}+m_{1}+2 m_{2}}}+(1 \leftrightarrow 2)\right)\right],
\end{aligned}
$$

where we are using the shorthand $(1.2)$ for $h_{i j, k}$ and the term $(1 \leftrightarrow 2)$ means the same as the previous one except with the subscript switch $(1 \leftrightarrow 2)$ for all variables $\left(W_{i}, h_{i}, m_{i}\right)$ in the summand. The origin of the three terms in (A.14), each of which contains a doubly-infinite sum, is a two-fold Mellin-Barnes integral precisely of the form worked out in ref. [56, eq. (B.40)] which makes an appearance in an intermediate step. For present purposes, we are only interested in the first term, since that is the one which will correspond to the exchange of single-trace operators in the intermediate channels of the conformal block. This determination is made by comparing the exponents of the position-dependent factors in the integrand with the expected dependence obtained from the analogous $p$-adic conformal block [56]. Thus we drop the second and third terms in (A.14).

Looking forward, the main remaining task is to evaluate the $s, t, \nu$ contour integrals in (A.14) and (A.10) and obtain a candidate holographic representation for the conformal block. In practice, it suffices to obtain an expression which is proportional to the holographic representation up to some overall constants, which can then be fixed by a boundary condition such as an OPE limit. To do this, we first close the $s$-plane contour to the left and pick up appropriate poles, followed by closing the $t$-plane contour to the right. We need only focus on the residues at the following poles: $\left\{s=-t-h_{4}-m_{3}, t=m_{4}\right\}$ for all $m_{3}, m_{4} \in \mathbb{Z}^{\geq 0}$, as this choice suffices for the present purposes. Finally, we evaluate the residue in the $\nu$-plane at $\nu=d / 2-\Delta_{\delta_{2}}$, which leads to a candidate conformal block, up to some possible proportionality constant to be determined. Carrying out these steps, we end up with

$$
\begin{aligned}
& A \supset 4 \frac{C_{\Delta_{1} \Delta_{2} \Delta_{\delta_{1}}} C_{\Delta_{5} \Delta_{6} \Delta_{\delta_{3}}} C_{\Delta_{\delta_{1}} \Delta_{3} \Delta_{\delta_{2}}} C_{\Delta_{\delta_{3}} \Delta_{4} \Delta_{\delta_{2}}}}{B\left(\Delta_{\delta_{1} 1,2}, \Delta_{\delta_{1} 2,1}\right) B\left(\Delta_{\delta_{3} 5,6}, \Delta_{\delta_{3} 6,5}\right)} \\
& \times \sum_{\substack{m_{1}, m_{2}, m_{3}, m_{4} \\
k_{1}, k_{3}=0}}^{\infty} \frac{(-1)^{m_{1}+m_{2}+m_{3}+m_{4}}}{k_{1} ! k_{3} ! m_{1} ! m_{2} ! m_{3} ! m_{4} !} \frac{(-1)^{m_{1}-m_{2}-m_{4}}}{4} \\
& \times \frac{B\left(\Delta_{\delta_{2}}, \Delta_{\delta_{1} \delta_{3}, 34}\right)}{B\left(\Delta_{\delta_{1} \delta_{2}, 3}, \Delta_{\delta_{3} \delta_{2}, 4}\right)} \frac{\left(\Delta_{3 \delta_{2}, \delta_{1}}\right)_{-k_{1}-m_{1,234}}\left(\Delta_{4 \delta_{2}, \delta_{3}}\right)_{-k_{3}+m_{34},}\left(\Delta_{\delta_{1} \delta_{3}, 34}\right)_{k_{13},+m_{12,3}}}{\left(\Delta_{\delta_{1}}-d / 2+1\right)_{k_{1}}\left(\Delta_{\delta_{2}}-d / 2+1\right)_{-m_{1,24}}\left(\Delta_{\delta_{3}}-d / 2+1\right)_{k_{3}}}
\end{aligned}
$$

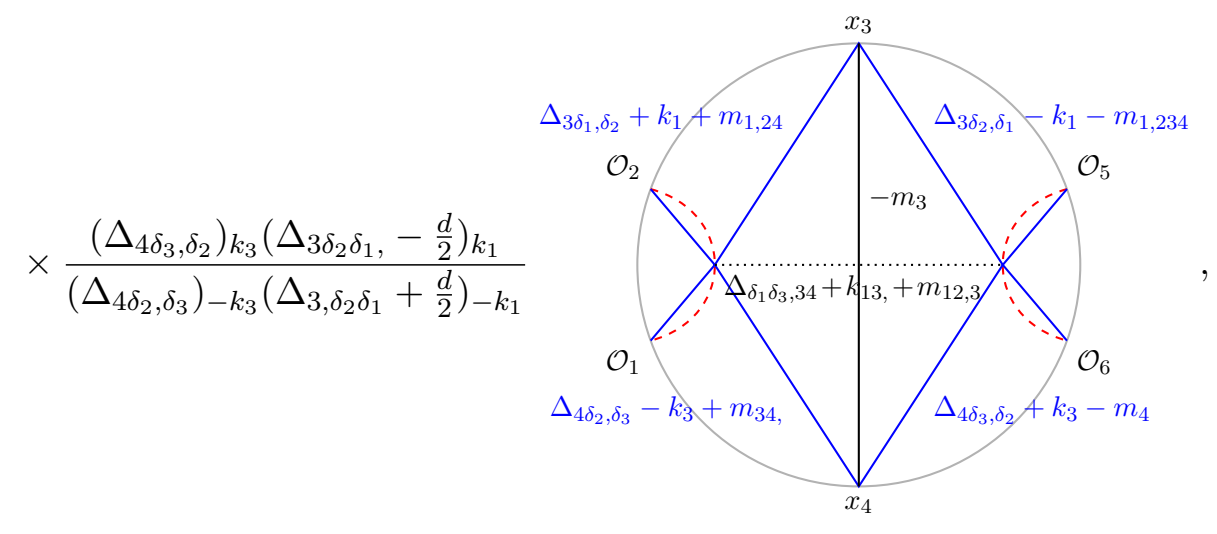


where we are using the shorthand (1.3) for $m_{a b, c}, k_{a b, c}$ and are now fully implementing the diagrammatic notation described in figure 2 (including the color-coding).

It turns out, two of the six infinite sums above can be re-expressed in closed-form. To see this, we first need to do the following series of change of variables: $m_{4} \rightarrow m_{4}-m_{2}$, followed by $m_{1} \rightarrow m_{1}-k_{1}$ and $m_{2} \rightarrow m_{2}-k_{3}$. The lower limits of the new variables can be shifted back to 0 with impunity. This leaves us with two coordinate-independent hypergeometric sums over $k_{1}$ and $k_{3}$, which readily lead to the appearance of two $m_{i^{-}}$ dependent factors of the generalized hypergeometric function ${ }_{3} F_{2} \cdot{ }^{22}$ At this point, comparison with (2.5)-(2.6) leads to a candidate holographic representation for the block. In fact it can be checked that it satisfies all appropriate conformal Casimir equations. To prove this is indeed a conformal block, one also needs to make sure the OPE limits are satisfied. Indeed it turns out to be the case, up to some overall position-independent normalization. The easiest way to fix the overall normalization is by requiring that the object have the right double-OPE limit to the well-known power-series expansion for the four-point block. See section 2.2. This leads to the introduction of an overall factor of

$$
\frac{4 B\left(\Delta_{\delta_{1} \delta_{2}, 3}, \Delta_{\delta_{3} \delta_{2}, 4}\right) B\left(1-\Delta_{\delta_{1} \delta_{2}, 3}, 1-\Delta_{\delta_{3} \delta_{2}, 4}\right)}{B\left(\Delta_{\delta_{2}}, \Delta_{\delta_{1} \delta_{3}, 34}\right) B\left(1-\Delta_{\delta_{2}}, 1-\Delta_{\delta_{1} \delta_{3}, 34}\right)}=\frac{4 B\left(\Delta_{\delta_{1} \delta_{2}, 3}, \Delta_{\delta_{3} \delta_{2}, 4}\right)}{B\left(\Delta_{\delta_{2}}, \Delta_{\delta_{1} \delta_{3}, 34}\right)} \lambda_{6}
$$

on the r.h.s. of (A.15), where $\lambda_{6}$ is defined in (2.9). The object thus obtained is the holographic dual of the six-point conformal block, and is given in (2.7)-eqrefc6Def.

\section{B Five- and seven-point examples}

In this appendix we briefly discuss the holographic representation of the seven-point comb channel block and its double-OPE limit, which leads to a power series expansion for the five-point block. The holographic representations (2.2) and (2.7) for the five- and six-point cases inform the following conjecture for the seven-point block (which is consistent with the general conjecture (3.1)-(3.4)):

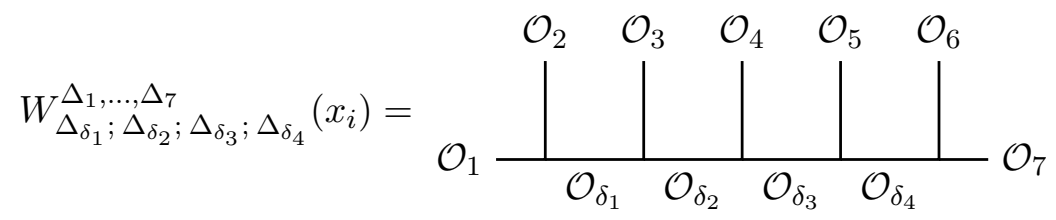

$$
\begin{aligned}
& =\frac{4}{B\left(\Delta_{\delta_{1} 1,2}, \Delta_{\delta_{1} 2,1}\right) B\left(\Delta_{\delta_{4} 6,7}, \Delta_{\delta_{4} 7,6}\right)} \mathcal{W}_{\Delta_{\delta_{1}} ; \Delta_{\delta_{2}} ; \Delta_{\delta_{3}} ; \Delta_{\delta_{4}}}^{\Delta_{1}, \ldots, \Delta_{i}}\left(x_{i}\right),
\end{aligned}
$$

\footnotetext{
${ }^{22}$ In fact at this point one can use a functional identity for the hypergeometric function [94] to recast the summand into a form very familiar from the case of the five-point conformal block [55].
} 
where the linear combination of seven-point geodesic bulk diagrams $\mathcal{W}$ is given by

$$
\mathcal{W}_{\Delta_{\delta_{1}} ; \Delta_{\delta_{2}} ; \Delta_{\delta_{3}} ; \Delta_{\delta_{4}}}^{\Delta_{1}, \ldots, \Delta_{7}}\left(x_{i}\right)=\sum_{\substack{k_{1}, k_{2}, k_{3}, k_{4}, j_{1}, j_{2}, j_{3}=0}}^{\infty} c_{k_{1}, k_{2}, k_{3}, k_{4} ; j_{1}, j_{2}, j_{3}}^{\Delta_{\delta_{1}}, \Delta_{\delta_{2}}, \Delta_{\delta_{3}}, \Delta_{\delta_{4}} ; \Delta_{3}, \Delta_{4}, \Delta_{5}}
$$

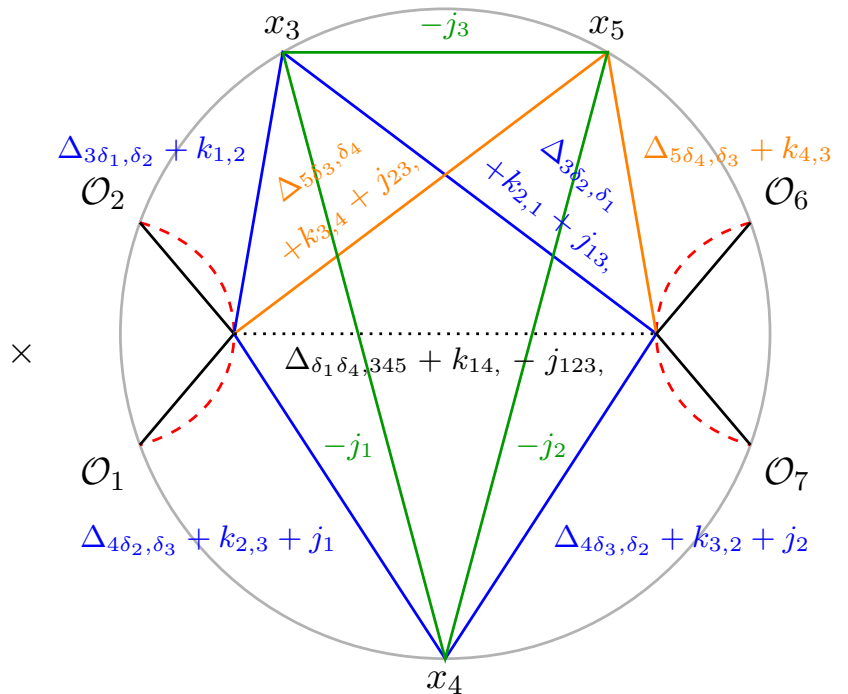

with the coefficients

$$
\begin{aligned}
& c_{k_{1}, k_{2},}^{\Delta_{\delta_{1}}, k_{3}, k_{4} ; j_{1}, j_{2}, j_{3}}, \Delta_{\delta_{3}}, \Delta_{\delta_{3}} ; \Delta_{3}, \Delta_{4}, \Delta_{5} \\
& \equiv \lambda_{7} \frac{(-1)^{k_{1}+k_{4}+j_{1}+j_{2}+j_{3}} \frac{\left(1-\Delta_{3 \delta_{2}, \delta_{1}}\right)_{k_{1}}\left(1-\Delta_{3 \delta_{1}, \delta_{2}}\right)_{k_{2}}}{\left(\Delta_{\delta_{1}}-d / 2+1\right)_{k_{1}}}}{k_{1} ! k_{2} ! k_{3} ! k_{4} ! j_{1} ! j_{2} ! j_{3} !} \frac{\left(1-\Delta_{4 \delta_{3}, \delta_{2}}\right)_{k_{2}}\left(1-\Delta_{4 \delta_{2}, \delta_{3}}\right)_{k_{3}}\left(1-\Delta_{5 \delta_{4}, \delta_{3}}\right)_{k_{3}}\left(1-\Delta_{5 \delta_{3}, \delta_{4}}\right)_{k_{4}}}{\left(\Delta_{\delta_{2}}-d / 2+1\right)_{k_{2}}\left(\Delta_{\delta_{3}}-d / 2+1\right)_{k_{3}}\left(\Delta_{\delta_{4}}-d / 2+1\right)_{k_{4}}} \\
& \quad \times \frac{\left(\Delta_{3 \delta_{1}, \delta_{2}}\right)_{k_{1,2}}\left(\Delta_{3 \delta_{2}, \delta_{1}}\right)_{k_{2,1}+j_{13},}\left(\Delta_{4 \delta_{2}, \delta_{3}}\right)_{k_{2,3}+j_{1}}\left(\Delta_{4 \delta_{3}, \delta_{2}}\right)_{k_{3,2}+j_{2}}}{} \\
& \quad \times\left(\Delta_{5 \delta_{3}, \delta_{4}}\right)_{k_{3,4}+j_{23},}\left(\Delta_{5 \delta_{4}, \delta_{3}}\right)_{k_{4}, 3}\left(\Delta_{\delta_{1} \delta_{4}, 345}\right)_{k_{14},-j_{123},} \\
& \quad \times{ }_{3} F_{2}\left[\left\{-k_{1},-k_{2}, \Delta_{\delta_{1} \delta_{2} 3,}-d / 2\right\} ;\left\{\Delta_{3 \delta_{2}, \delta_{1}}-k_{1}, \Delta_{3 \delta_{1}, \delta_{2}}-k_{2}\right\} ; 1\right] \\
& \quad \times{ }_{3} F_{2}\left[\left\{-k_{2},-k_{3}, \Delta_{\delta_{2} \delta_{3} 4,}-d / 2\right\} ;\left\{\Delta_{4 \delta_{3}, \delta_{2}}-k_{2}, \Delta_{4 \delta_{2}, \delta_{3}}-k_{3}\right\} ; 1\right] \\
& \quad \times{ }_{3} F_{2}\left[\left\{-k_{3},-k_{4}, \Delta_{\delta_{3} \delta_{4} 5,}-d / 2\right\} ;\left\{\Delta_{5 \delta_{4}, \delta_{3}}-k_{3}, \Delta_{5 \delta_{3}, \delta_{4}}-k_{4}\right\} ; 1\right],
\end{aligned}
$$

where

$$
\lambda_{7} \equiv \frac{\Gamma\left(1-\Delta_{\delta_{1} \delta_{2}, 3}\right) \Gamma\left(1-\Delta_{\delta_{2} \delta_{3}, 4}\right) \Gamma\left(1-\Delta_{\delta_{3} \delta_{4}, 5}\right)}{\Gamma\left(1-\Delta_{\delta_{1} \delta_{4}, 345}\right) \Gamma\left(1-\Delta_{\delta_{2}}\right) \Gamma\left(1-\Delta_{\delta_{3}}\right)} .
$$

Here we are using the subscript convention (1.3) for both $k$ and $j$ integral parameters. In drawing the geodesic diagram above, we have eschewed the color-coding scheme prescribed in figure 2 to help guide the eye. It should be clear from the diagram which lines represent a bulk-to-boundary propagator, and which lines represent purely boundary contractions of the form $\left(x_{i j}^{2}\right)^{-\Delta}$. The factor of chordal distance continues to be shown as a dotted black line. 
To prove this conjecture, we must show that (B.1) satisfies the conformal Casimir equations (1.7) for $n=7$ and $K=2,3,4,5$. Due to the symmetry of the object, we need only check the cases $K=2,3$; the remaining two cases follow trivially after relabelling. Further, we must show the OPE limit (1.8). As remarked in section 2.1, the conformal Casimir check is in fact straightforward though lengthy to work out, but the procedure is identical to the ones described in refs. $[55,56]$ in the context of five- and six-point blocks. No new ingredients are needed, except for a triple-application of the hypergeometric identity (2.11), once for each factor of the ${ }_{3} F_{2}$ function in (B.3). Similarly, the object obtained in the OPE limit can be shown to be an alternate holographic representation of the six-point block involving a single geodesic integral, via a similar proof by Casimir. So to keep the paper to a reasonable length, we will refrain from providing the somewhat lengthy details here. In lieu of this, we provide a conformal Casimir check of the series expansion of the seven-point block in section 3. The OPE limits of the six-point block obtained above themselves lead to two different representations of the five-point block - one corresponding to a holographic representation involving a single geodesic integral similar to (2.14), with the other more interesting limit producing a power series expansion for the five-point block as discussed next.

\section{B.1 Double-OPE limit and the five-point block}

Consider the following double-OPE limit of the seven-point block,

$$
\begin{aligned}
& W_{\Delta_{\delta_{1}} ; \Delta_{\delta_{2}} ; \Delta_{\delta_{3}} ; \Delta_{\delta_{4}}}^{\Delta_{1}, \ldots, \Delta_{7}}\left(x_{1}, \ldots, x_{7}\right) \\
& \stackrel{\substack{x_{2} \rightarrow x_{1} \\
x_{7} \rightarrow x_{6}}}{\longrightarrow}\left(x_{12}^{2}\right)^{\Delta_{\delta_{1}, 12}}\left(x_{67}^{2}\right)^{\Delta_{\delta_{4}, 67}} \sum_{\substack{k_{2}, k_{3}, j_{1}, j_{2}, j_{3}=0}}^{\infty} c_{0, k_{2}, k_{3}, 0 ; j_{1}, j_{2}, j_{3}}^{\Delta_{\delta_{1}}, \Delta_{\delta_{2}}, \Delta_{\delta_{3}}, \Delta_{\delta_{4}} ; \Delta_{3}, \Delta_{4}, \Delta_{5}}
\end{aligned}
$$

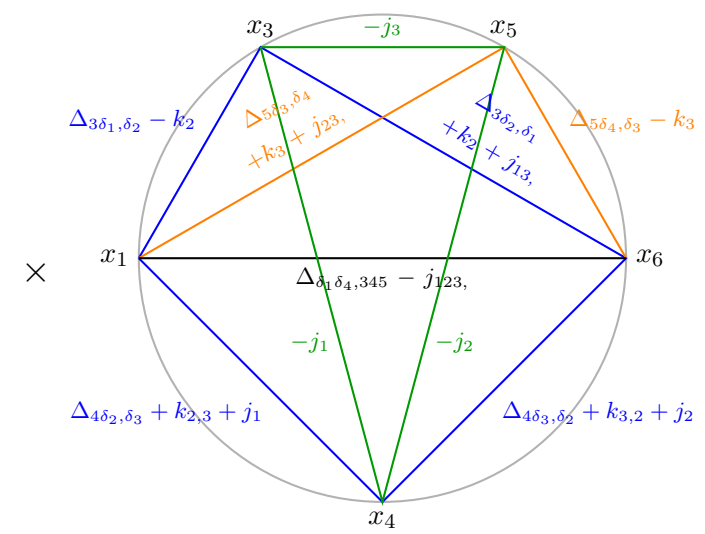

$$
\begin{aligned}
& \equiv\left(x_{12}^{2}\right)^{\Delta_{\delta_{1}, 12}}\left(x_{67}^{2}\right)^{\Delta_{\delta_{4}, 67}} \tilde{V} .
\end{aligned}
$$

The claim is that $\widetilde{V}$ is a (power series expansion of the) five-point block. Explicitly, we may write it as

$$
\begin{aligned}
\widetilde{V}= & W_{0}^{\Delta_{\delta_{1}}, \Delta_{3}, \Delta_{4}, \Delta_{5}, \Delta_{\delta_{4}}}\left(x_{1}, x_{3}, x_{4}, x_{5}, x_{6}\right) \\
& \times u_{1}^{\frac{\Delta_{\delta_{2}}}{2}} u_{2}^{\frac{\Delta_{\delta_{3}}}{2}} \sum_{\substack{k_{2}, k_{3}, j_{1}, j_{2}, j_{3}=0}}^{\infty} c_{0, k_{2}, k_{3}, 0 ; j_{1}, j_{2}, j_{3}}^{\Delta_{\delta_{1}}, \Delta_{\delta_{2}}, \Delta_{\delta_{3}}, \Delta_{\delta_{4}} ; \Delta_{3}, \Delta_{4}, \Delta_{5}} u_{1}^{k_{2}} u_{2}^{k_{3}} v_{1}^{j_{1}} v_{2}^{j_{2}} w^{j_{3}},
\end{aligned}
$$


where the leg factor $W_{0}^{\Delta_{\delta_{1}}, \Delta_{3}, \Delta_{4}, \Delta_{5}, \Delta_{\delta_{4}}}\left(x_{1}, x_{3}, x_{4}, x_{5}, x_{6}\right)$ was defined in (1.5), ${ }^{23}$ and we have defined the cross-ratios

$$
u_{1} \equiv \frac{x_{13}^{2} x_{46}^{2}}{x_{14}^{2} x_{36}^{2}} \quad v_{1} \equiv \frac{x_{16}^{2} x_{34}^{2}}{x_{14}^{2} x_{36}^{2}} \quad u_{2} \equiv \frac{x_{14}^{2} x_{56}^{2}}{x_{15}^{2} x_{46}^{2}} \quad v_{2} \equiv \frac{x_{16}^{2} x_{45}^{2}}{x_{15}^{2} x_{46}^{2}} \quad w \equiv \frac{x_{16}^{2} x_{35}^{2}}{x_{15}^{2} x_{36}^{2}} .
$$

Writing out the coefficients explicitly, we get

$$
\begin{aligned}
& \begin{array}{l}
\Delta_{\delta_{1}}, \Delta_{\delta_{2}}, \Delta_{\delta_{3}}, \Delta_{\delta_{4}} ; \Delta_{3}, \Delta_{4}, \Delta_{5} \\
c_{0, k_{2}, k_{3}, 0 ; j_{1}, j_{2}, j_{3}}
\end{array} \\
& =\lambda_{7} \frac{(-1)^{k_{2}+k_{3}+j_{1}+j_{2}+j_{3}}}{k_{2} ! k_{3} ! j_{1} ! j_{2} ! j_{3} !} \frac{\left(1-\Delta_{4 \delta_{3}, \delta_{2}}\right)_{k_{2}}\left(1-\Delta_{4 \delta_{2}, \delta_{3}}\right)_{k_{3}}}{\left(\Delta_{\delta_{2}}-d / 2+1\right)_{k_{2}}\left(\Delta_{\delta_{3}}-d / 2+1\right)_{k_{3}}} \\
& \times\left(\Delta_{3 \delta_{2}, \delta_{1}}\right)_{k_{2}+j_{13},}\left(\Delta_{4 \delta_{2}, \delta_{3}}\right)_{k_{2,3}+j_{1}}\left(\Delta_{4 \delta_{3}, \delta_{2}}\right)_{k_{3,2}+j_{2}} \\
& \times\left(\Delta_{5 \delta_{3}, \delta_{4}}\right)_{k_{3}+j_{23},}\left(\Delta_{\delta_{1} \delta_{4}, 345}\right)_{-j_{123},} \\
& \times{ }_{3} F_{2}\left[\left\{-k_{2},-k_{3}, \Delta_{\delta_{2} \delta_{3} 4},-d / 2\right\} ;\left\{\Delta_{4 \delta_{3}, \delta_{2}}-k_{2}, \Delta_{4 \delta_{2}, \delta_{3}}-k_{3}\right\} ; 1\right],
\end{aligned}
$$

where $\lambda_{7}$ was given in (B.4). It can be checked that this is just a rewriting of (1.1) for $n=5$ in different variables. The proof that this is indeed a five-point block is given in section 3 .

\section{Further technical details}

\section{C.1 Proof of (3.15)}

In this section we will prove (3.15). Consider first the $j_{\langle 2 \mid n-1\rangle}$ sum on the right hand side. We can use the following identity to evaluate this sum:

$$
\sum_{k=0}^{\infty} \frac{(-1)^{k}}{k !}(a)_{k}(b)_{\ell_{1}+k}(c)_{-\ell_{2}-k}=\frac{(-1)^{\ell_{2}} \Gamma(1-c) \Gamma\left(b+\ell_{1}\right) \Gamma\left(-a-b-c-\ell_{1}+\ell_{2}+1\right)}{\Gamma(b) \Gamma\left(-a-c+\ell_{2}+1\right) \Gamma\left(-b-c-\ell_{1}+\ell_{2}+1\right)},
$$

which holds for integers $\ell_{1}, \ell_{2}$, and we assume convergence of the sum. Performing the sum using the identity above, the right hand side of (3.15) becomes

$$
\begin{aligned}
\text { r.h.s. }= & \frac{\Gamma\left(1-\Delta_{\delta_{n-3} n,(n-1)}\right) \Gamma\left(1-\Delta_{\delta_{1} \delta_{n-3}, 3 \ldots(n-2)}\right)}{\Gamma\left(1-\Delta_{\delta_{1} n, 3 \ldots(n-1)}\right) \Gamma\left(1-\Delta_{\delta_{n-3}}\right)} \sum_{\substack{j_{\langle 3 \mid n-1\rangle}, \ldots . \\
j_{\langle n-2 \mid n-1\rangle}=0}}^{\infty}\left(\prod_{\ell=3}^{n-2} \frac{(-1)^{j_{\langle\ell \mid n-1\rangle}}}{j_{\langle\ell \mid n-1\rangle} !}\right) \\
& \times\left(\prod_{t=1}^{n-4}\left(\Delta_{(t+2) \delta_{t+1}, \delta_{t}}+k_{t+1, t}+\sum_{s=t+3}^{n-2} j_{\langle t+2 \mid s\rangle}\right)\right. \\
& \times\left(\Delta_{(n-1) \delta_{n-3}, n}\right)_{\sum_{r=3}^{n-2} j_{\langle r \mid n-1\rangle}} \frac{\left(\Delta_{\delta_{1} n, 3 \ldots(n-1)}+k_{1}\right)_{-\sum_{3 \leq r<s \leq n-1} j_{\langle r \mid s\rangle}}}{\left(\Delta_{\delta_{1} \delta_{n-3}, 3 \ldots(n-2)}+k_{1}\right)_{-\sum_{3 \leq r<s \leq n-2} j_{\langle r \mid s\rangle}}},
\end{aligned}
$$

\footnotetext{
${ }^{23} \mathrm{An}$ alternate way to write the leg factor is as follows:

$W_{0}^{\Delta_{\delta_{1}}, \Delta_{3}, \Delta_{4}, \Delta_{5}, \Delta_{\delta_{4}}}\left(x_{1}, x_{3}, x_{4}, x_{5}, x_{6}\right)=\frac{1}{\left(x_{13}^{2}\right)^{\Delta_{\delta_{1} 3},}\left(x_{46}^{2}\right)^{\frac{\Delta_{4}}{2}}\left(x_{56}^{2}\right)^{\Delta_{\delta_{4} 5}}}\left(\frac{x_{16}^{2}}{x_{36}^{2}}\right)^{\Delta_{3, \delta_{1}}}\left(\frac{x_{16}^{2}}{x_{14}^{2}}\right)^{\frac{\Delta_{4}}{2}}\left(\frac{x_{15}^{2}}{x_{16}^{2}}\right)^{\Delta_{\delta_{4}, 5}}$.
} 
where $\delta_{0}=\Delta_{1}, k_{0}=0$ and $k_{n-3}=0$ as before. Notice that (C.2) is of precisely the same form as the original sum on the right hand side of (3.15), except with one fewer sum. Thus one can iteratively apply (C.1) to systematically reduce r.h.s. further and further. For instance, performing the $j_{\langle 3 \mid n-1\rangle}$ sum next yields

$$
\begin{aligned}
r . h . s .= & \frac{\Gamma\left(1-\Delta_{\delta_{n-3} n,(n-1)}\right) \Gamma\left(1-\Delta_{\delta_{2} \delta_{n-3}, 4 \ldots(n-2)}\right)}{\Gamma\left(1-\Delta_{\delta_{2} n, 4 \ldots(n-1)}\right) \Gamma\left(1-\Delta_{\delta_{n-3}}\right)} \sum_{\substack{j_{\langle 4 \mid n-1\rangle}, \ldots, j_{\langle n-2 \mid n-1\rangle}=0}}^{\infty}\left(\prod_{\ell=4}^{n-2} \frac{(-1)^{j_{\langle\ell \mid n-1\rangle}}}{j_{\langle\ell \mid n-1\rangle} !}\right) \\
& \times\left(\prod_{t=2}^{n-4}\left(\Delta_{(t+2) \delta_{t+1}, \delta_{t}}+k_{t+1, t}+\sum_{s=t+3}^{n-2} j_{\langle t+2 \mid s\rangle}\right)\right. \\
& \times\left(\Delta_{(n-1) \delta_{n-3}, n}\right)_{\sum_{r=4}^{n-2} j_{\langle r \mid n-1\rangle}} \frac{\left(\Delta_{\delta_{2} n, 4 \ldots(n-1)}+k_{2}\right)_{-\sum_{4 \leq r<s \leq n-1} j_{\langle r \mid s\rangle}}}{\left(\Delta_{\delta_{2} \delta_{n-3}, 4 \ldots(n-2)}+k_{2}\right)_{-\sum_{4 \leq r<s \leq n-2} j_{\langle r \mid s\rangle}}}
\end{aligned}
$$

where the pattern should be clear by now. Reducing r.h.s. iteratively by performing the $j_{\langle 4 \mid n-1\rangle}, j_{\langle 5 \mid n-1\rangle}, \ldots$ sums in this order, it is straightforward to check that r.h.s. reduces to unity.

\section{C.2 Proof of (3.32)}

Define,

$$
J \equiv \sum_{1 \leq r<s \leq K} \sum_{\substack{a_{u}, a_{v}=1 \\ a_{u}, a_{v} \notin\{r, s\}, a_{u} \neq a_{v}}}^{K} \Delta_{\left\langle a_{u} \mid s\right\rangle} \Delta_{\left\langle a_{v} \mid r\right\rangle} \frac{\left(x_{r s}^{2} x_{a_{u} a_{v}}^{2}-x_{s a_{v}}^{2} x_{r a_{u}}^{2}\right)}{x_{s a_{u}}^{2} x_{r a_{v}}^{2}}
$$

We will show that $J=0$, which proves (3.32). First expand $J$ as

$$
\begin{aligned}
J & =\sum_{1 \leq r<s \leq K}\left(\sum_{\substack{1 \leq a_{u}<a_{v} \leq K \\
a_{u}, a_{v} \notin\{r, s\}}}+\sum_{\substack{1 \leq a_{v}<a_{u} \leq K \\
a_{u}, a_{v} \notin\{r, s\}}}\right) \Delta_{\left\langle a_{u} \mid s\right\rangle} \Delta_{\left\langle a_{v} \mid r\right\rangle} \frac{\left(x_{r s}^{2} x_{a_{u} a_{v}}^{2}-x_{s a_{v}}^{2} x_{r a_{u}}^{2}\right)}{x_{s a_{u}}^{2} x_{r a_{v}}^{2}} \\
& =\left(\sum_{1 \leq r<s \leq K}+\sum_{1 \leq s<r \leq K}\right) \sum_{\substack{1 \leq a_{u}<a_{v} \leq K \\
a_{u}, a_{v} \notin\{r, s\}}} \Delta_{\left\langle a_{u} \mid s\right\rangle} \Delta_{\left\langle a_{v} \mid r\right\rangle} \frac{\left(x_{r s}^{2} x_{a_{u} a_{v}}^{2}-x_{s a_{v}}^{2} x_{r a_{u}}^{2}\right)}{x_{s a_{u}}^{2} x_{r a_{v}}^{2}} \\
& =\left(J_{1}+J_{2}+J_{3}+J_{4}+J_{5}+J_{6}\right)+\left(J_{7}+J_{8}+J_{9}+J_{10}+J_{11}+J_{12}\right),
\end{aligned}
$$

where we have broken the four-fold sums into the following constituent pieces:

$$
\begin{aligned}
J_{1} & \equiv \sum_{1 \leq a_{u}<a_{v}<r<s \leq K} \Delta_{\left\langle a_{u} \mid s\right\rangle} \Delta_{\left\langle a_{v} \mid r\right\rangle} \frac{\left(x_{r s}^{2} x_{a_{u} a_{v}}^{2}-x_{s a_{v}}^{2} x_{r a_{u}}^{2}\right)}{x_{s a_{u}}^{2} x_{r a_{v}}^{2}} \\
J_{2} & \equiv \sum_{1 \leq a_{u}<r<a_{v}<s \leq K} \Delta_{\left\langle a_{u} \mid s\right\rangle} \Delta_{\left\langle a_{v} \mid r\right\rangle} \frac{\left(x_{r s}^{2} x_{a_{u} a_{v}}^{2}-x_{s a_{v}}^{2} x_{r a_{u}}^{2}\right)}{x_{s a_{u}}^{2} x_{r a_{v}}^{2}} \\
J_{3} & \equiv \sum_{1 \leq a_{u}<r<s<a_{v} \leq K} \Delta_{\left\langle a_{u} \mid s\right\rangle} \Delta_{\left\langle a_{v} \mid r\right\rangle} \frac{\left(x_{r s}^{2} x_{a_{u} a_{v}}^{2}-x_{s a_{v}}^{2} x_{r a_{u}}^{2}\right)}{x_{s a_{u}}^{2} x_{r a_{v}}^{2}}
\end{aligned}
$$




$$
\begin{aligned}
J_{4} & \equiv \sum_{1 \leq r<a_{u}<a_{v}<s \leq K} \Delta_{\left\langle a_{u} \mid s\right\rangle} \Delta_{\left\langle a_{v} \mid r\right\rangle} \frac{\left(x_{r s}^{2} x_{a_{u} a_{v}}^{2}-x_{s a_{v}}^{2} x_{r a_{u}}^{2}\right)}{x_{s a_{u}}^{2} x_{r a_{v}}^{2}} \\
J_{5} & \equiv \sum_{1 \leq r<a_{u}<s<a_{v} \leq K} \Delta_{\left\langle a_{u} \mid s\right\rangle} \Delta_{\left\langle a_{v} \mid r\right\rangle} \frac{\left(x_{r s}^{2} x_{a_{u} a_{v}}^{2}-x_{s a_{v}}^{2} x_{r a_{u}}^{2}\right)}{x_{s a_{u}}^{2} x_{r a_{v}}^{2}} \\
J_{6} & \equiv \sum_{1 \leq r<s<a_{u}<a_{v} \leq K} \Delta_{\left\langle a_{u} \mid s\right\rangle} \Delta_{\left\langle a_{v} \mid r\right\rangle} \frac{\left(x_{r s}^{2} x_{a_{u} a_{v}}^{2}-x_{s a_{v}}^{2} x_{r a_{u}}^{2}\right)}{x_{s a_{u}}^{2} x_{r a_{v}}^{2}}
\end{aligned}
$$

and

$$
\begin{aligned}
J_{7} & \equiv \sum_{1 \leq a_{u}<a_{v}<s<r \leq K} \Delta_{\left\langle a_{u} \mid s\right\rangle} \Delta_{\left\langle a_{v} \mid r\right\rangle} \frac{\left(x_{r s}^{2} x_{a_{u} a_{v}}^{2}-x_{s a_{v}}^{2} x_{r a_{u}}^{2}\right)}{x_{s a_{u}}^{2} x_{r a_{v}}^{2}} \\
J_{8} & \sum_{1 \leq a_{u}<s<a_{v}<r \leq K} \Delta_{\left\langle a_{u} \mid s\right\rangle} \Delta_{\left\langle a_{v} \mid r\right\rangle} \frac{\left(x_{r s}^{2} x_{a_{u} a_{v}}^{2}-x_{s a_{v}}^{2} x_{r a_{u}}^{2}\right)}{x_{s a_{u}}^{2} x_{r a_{v}}^{2}} \\
J_{9} & \equiv \sum_{1 \leq a_{u}<s<r<a_{v} \leq K} \Delta_{\left\langle a_{u} \mid s\right\rangle} \Delta_{\left\langle a_{v} \mid r\right\rangle} \frac{\left(x_{r s}^{2} x_{a_{u} a_{v}}^{2}-x_{s a_{v}}^{2} x_{r a_{u}}^{2}\right)}{x_{s a_{u}}^{2} x_{r a_{v}}^{2}} \\
J_{10} & \equiv \sum_{1 \leq s<a_{u}<a_{v}<r \leq K} \Delta_{\left\langle a_{u} \mid s\right\rangle} \Delta_{\left\langle a_{v} \mid r\right\rangle} \frac{\left(x_{r s}^{2} x_{a_{u} a_{v}}^{2}-x_{s a_{v}}^{2} x_{r a_{u}}^{2}\right)}{x_{s a_{u}}^{2} x_{r a_{v}}^{2}} \\
J_{11} & \equiv \sum_{1 \leq s<a_{u}<r<a_{v} \leq K} \Delta_{\left\langle a_{u} \mid s\right\rangle} \Delta_{\left\langle a_{v} \mid r\right\rangle} \frac{\left(x_{r s}^{2} x_{a_{u} a_{v}}^{2}-x_{s a_{v}}^{2} x_{r a_{u}}^{2}\right)}{x_{s a_{u}}^{2} x_{r a_{v}}^{2}} \\
J_{12} & \equiv \sum_{1 \leq s<r<a_{u}<a_{v} \leq K} \Delta_{\left\langle a_{u} \mid s\right\rangle} \Delta_{\left\langle a_{v} \mid r\right\rangle} \frac{\left(x_{r s}^{2} x_{a_{u} a_{v}}^{2}-x_{s a_{v}}^{2} x_{r a_{u}}^{2}\right)}{x_{s a_{u}}^{2} x_{r a_{v}}^{2}} .
\end{aligned}
$$

Now, observe that switching the dummy variables $r \leftrightarrow a_{v}$ in $J_{2}$ turns it manifestly into $-J_{1}$. Likewise, doing the dummy variable replacement $s \leftrightarrow a_{u}$ in $J_{6}$ turns it precisely to $-J_{5}$, and the switches $r \leftrightarrow a_{u}, s \leftrightarrow a_{v}$ turn $J_{4}$ into $J_{3}$. Similarly, switching $s \leftrightarrow a_{u}$ turns $J_{10}$ manifestly into $-J_{8}$, and $J_{11}$ into $-J_{9}$. Finally, switching $s \leftrightarrow a_{u}, r \leftrightarrow a_{v}$ turns $J_{12}$ into $J_{7}$. Thus, we conclude $J=2 J_{3}+2 J_{7}$. However, making the dummy variable switch $r \leftrightarrow a_{v}$ turns $J_{7}$ into $-J_{3}$. Thus, $J$ vanishes identically.

\section{C.3 Proof of (3.37)}

In this subsection we will show the details of the calculation leading from (3.35)-(3.36) to (3.37). After integer shifting the integral parameters to get all terms in (3.35) to have uniform position dependence as explained below (3.36), we re-express (3.35) as

$$
\begin{aligned}
\text { l.h.s. }= & W_{0}^{(n)}\left(x_{i}\right)\left(\prod_{i=1}^{n-3} u_{i}^{\frac{\Delta_{\delta_{i}}}{2}}\right) \\
& \times \sum_{\substack{k_{1}, \ldots, k_{n-3}, j_{\langle 2 \mid 3\rangle}, j_{\langle 2 \mid 4\rangle}, \ldots, j_{\langle n-2 \mid n-1\rangle}}}\left(\prod_{i=1}^{n-3} u_{i}^{k_{i}}\right)\left(\prod_{2 \leq r<s \leq n-1} w_{r ; s}^{j_{\langle>s\rangle}}\right)\left[c_{(\cdot)} \sum_{i=1}^{K} m_{\Delta_{i}}^{2}\right.
\end{aligned}
$$




$$
\begin{aligned}
& -2 c_{(\cdot)} \sum_{1 \leq r<s \leq K}\left(m_{\Delta_{\langle r \mid s\rangle}}^{2}+\Delta_{\langle r \mid s\rangle} \sum_{\ell=1}^{n-2}\left(\Delta_{\left\langle r \mid a_{\ell}\right\rangle}+\Delta_{\left\langle s \mid a_{\ell}\right\rangle}\right)-\sum_{\ell=1}^{n-2} \Delta_{\left\langle r \mid a_{\ell}\right\rangle} \Delta_{\left\langle s \mid a_{\ell}\right\rangle}\right) \\
& -2 \sum_{\substack{1 \leq r<s \leq K \\
K+1 \leq a_{u}<a_{v} \leq n-1}}\left(\widetilde{c}_{\left(k_{s-1}, s ; a_{u}, a_{v}\right.} \sum_{\left.k_{s}-1, \ldots, k_{a_{u}-2}-1, j_{\langle r \mid s\rangle}-1, j_{\left\langle a_{u} \mid a_{v}\right\rangle}-1, j_{\left\langle s \mid a_{u}\right\rangle}+1, j_{\left\langle r \mid a_{v}\right\rangle}+1\right)}\right. \\
& \left.\left.-\widetilde{c}_{\left(j_{\left\langle s \mid a_{v}\right\rangle}^{r}, s ; a_{u}, a_{v}\right.}-1, j_{\left\langle r \mid a_{u}\right\rangle}-1, j_{\left\langle s \mid a_{u}\right\rangle}+1, j_{\left\langle r \mid a_{v}\right\rangle}+1\right)\right) \\
& -2 \sum_{\substack{1 \leq r<s \leq K \\
K+1 \leq a_{v}<a_{u} \leq n-1}}\left(\widetilde{c}_{\left(k_{s-1}-1, a_{u}, a_{u}, a_{v}\right.}^{\left.r, 1, \ldots, k_{a_{v}-2}-1, j_{\langle r \mid s\rangle}-1, j_{\left\langle a_{v} \mid a_{u}\right\rangle}-1, j_{\left\langle s \mid a_{u}\right\rangle}+1, j_{\left\langle r \mid a_{v}\right\rangle}+1\right)}{ }_{\sim r, s ; a_{u}, a_{v}}\right. \\
& \left.-\widetilde{c}_{\left(j_{\left\langle s \mid a_{v}\right\rangle}^{r, s ; a_{u}, a_{v}}-1, j_{\left\langle r \mid a_{u}\right\rangle}-1, j_{\left\langle s \mid a_{u}\right\rangle}+1, j_{\left\langle r \mid a_{v}\right\rangle}+1\right)}\right)
\end{aligned}
$$

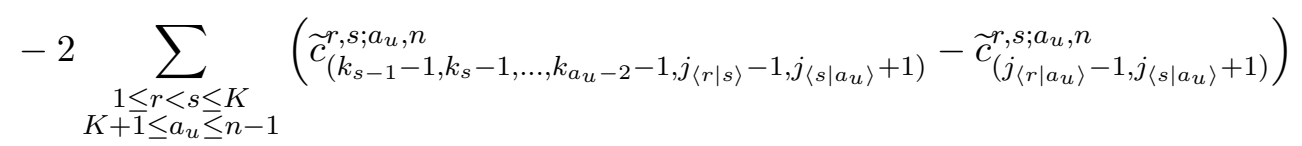

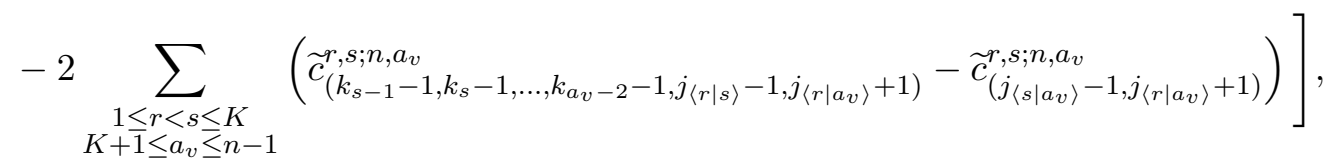

where we are utilizing the notation (3.38)-(3.39).

Thus for the above expression to equal (3.36) for all choices of boundary insertion points, each individual term in the sum must equal the conformal Casimir eigenvalue, times the coefficient (3.8). That is, the proof of (3.37) boils down to demonstrating the following non-trivial identity involving the coefficients,

$$
\begin{aligned}
& c_{(\cdot)}\left[\sum_{i=1}^{K} m_{\Delta_{i}}^{2}-2 \sum_{1 \leq r<s \leq K}\left(m_{\Delta_{\langle r \mid s\rangle}}^{2}+\Delta_{\langle r \mid s\rangle} \sum_{\ell=1}^{n-2}\left(\Delta_{\left\langle r \mid a_{\ell}\right\rangle}+\Delta_{\left\langle s \mid a_{\ell}\right\rangle}\right)\right.\right. \\
& \left.\left.-\sum_{\ell=1}^{n-2} \Delta_{\left\langle r \mid a_{\ell}\right\rangle} \Delta_{\left\langle s \mid a_{\ell}\right\rangle}\right)-m_{\Delta_{\delta_{K-1}}}^{2}\right] \\
& -2 \sum_{1 \leq r<s \leq K} \quad\left(\widetilde{c}_{\left(k_{s-1}-1, k_{s}-1, \ldots, k_{a_{u}-2}-1, j_{\langle r \mid s\rangle}-1, j_{\left\langle a_{u} \mid a_{v}\right\rangle}-1, j_{\left\langle s \mid a_{u}\right\rangle}+1, j_{\left\langle r \mid a_{v}\right\rangle}+1\right)}\right. \\
& K+1 \leq a_{u}<a_{v} \leq n-1 \quad-\widetilde{c}_{\left(j_{\left\langle s \mid a_{v}\right\rangle}^{r, s ; a_{u}, a_{v}}-1, j_{\left\langle r \mid a_{u}\right\rangle}-1, j_{\left\langle s \mid a_{u}\right\rangle}+1, j_{\left\langle r \mid a_{v}\right\rangle}+1\right)} \\
& +\widetilde{c}_{\left(k_{s-1}-1, k_{s}-1, \ldots, k_{a_{u}-2}-1, j_{\langle r \mid s\rangle}-1, j_{\left\langle a_{u} \mid a_{v}\right\rangle}, a_{u}, j_{\left\langle s \mid a_{v}\right\rangle}+1, j_{\left\langle r \mid a_{u}\right\rangle}+1\right)}
\end{aligned}
$$

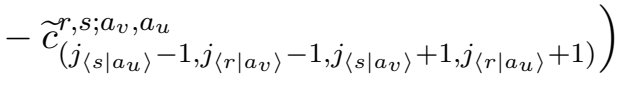

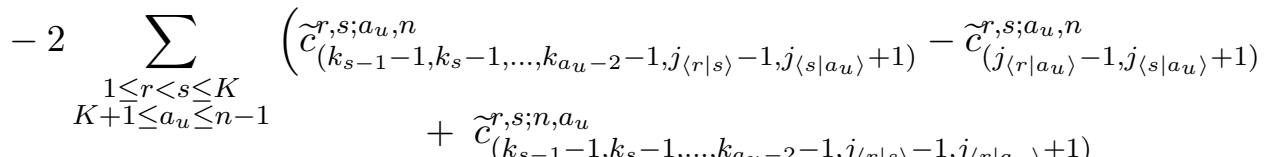

$$
\begin{aligned}
& \left.-\widetilde{c}_{\left(j_{\left\langle s \mid a_{u}\right\rangle}^{r}, s ; a_{u}-1, j_{\left\langle r \mid a_{u}\right\rangle}+1\right)}\right) \stackrel{!}{=} 0
\end{aligned}
$$

for $2 \leq K \leq n-2, n \geq 4$ and all parameters $k_{i}, j_{\langle\cdot \mid \cdot\rangle}$. 
We can use various symmetry properties of the scaled coefficients $\widetilde{c}$ to simplify (C.9). It is straightforward to check that for $1 \leq r<s \leq K$ and $K+1 \leq a_{u}<a_{v} \leq n-1$,

$$
\begin{aligned}
& \left.\widetilde{c}_{\left(j\left\langle s \mid a_{v}\right\rangle\right.}^{r, s ; a_{u}, a_{v}}-1, j_{\left\langle r \mid a_{u}\right\rangle}-1, j_{\left\langle s \mid a_{u}\right\rangle}+1, j_{\left\langle r \mid a_{v}\right\rangle}+1\right) \\
& =\widetilde{c}_{(\cdot)}^{r, s ; a_{v}, a_{u}} \\
& \left.\widetilde{c}_{\left(j, s ;\left|a_{u}\right\rangle\right.}^{r, s ; a_{v}, a_{u}}-1, j_{\left\langle r \mid a_{v}\right\rangle}-1, j_{\left\langle s \mid a_{v}\right\rangle}+1, j_{\left\langle r \mid a_{u}\right\rangle}+1\right) \\
& =\widetilde{c}_{(\cdot)}^{r, s ; a_{u}, a_{v}} \\
& \left.\widetilde{c}_{\left(k_{s-1}-1, k_{s}-1, \ldots, a_{u}, a_{v}\right.} k_{a_{u}-2}-1, j_{\langle r \mid s\rangle}-1, j_{\left\langle a_{u} \mid a_{v}\right\rangle}-1, j_{\left\langle s \mid a_{u}\right\rangle}+1, j_{\left\langle r \mid a_{v}\right\rangle}+1\right) \\
& =\widetilde{c}_{\left(k_{s-1}-1, k_{s}-1, \ldots, k_{a_{u}-2}-1, j_{\langle r \mid s\rangle}-1, j_{\left\langle a_{u} \mid a_{v}\right\rangle}-1, j_{\left\langle s \mid a_{v}\right\rangle}+1, j_{\left\langle r \mid a_{u}\right\rangle}+1\right)},
\end{aligned}
$$

and for $1 \leq r<s \leq K$ and $K+1 \leq a_{u} \leq n-1$,

$$
\begin{aligned}
\widetilde{c}_{\left(j_{\left\langle r \mid a_{u}\right\rangle},-1, j_{\left\langle s \mid a_{u}\right\rangle}+1\right)}^{r, s a_{u}, n} & =\widetilde{c}_{(\cdot)}^{s, r ; a_{u}, n} \\
\widetilde{c}_{\left(j_{\left\langle s \mid a_{u}\right\rangle}, r, n, a_{u}\right.}^{\left.r, j_{\left\langle r \mid a_{u}\right\rangle}+1\right)} & =\widetilde{c}_{(\cdot)}^{r, s ; a_{u}, n} \\
\widetilde{c}_{\left(k_{s-1}-1, k_{s}-1, \ldots, k_{a_{u}-2}-1, j_{\langle r \mid s\rangle}-1, j_{\left\langle s \mid a_{u}\right\rangle}+1\right)}^{r, s ; a_{u}, n} & =\widetilde{c}_{\left(k_{s-1}, s ; n, a_{u}\right.}^{\left.r, 1, k_{s}-1, \ldots, k_{a_{u}-2}-1, j_{\langle r \mid s\rangle}-1, j_{\left\langle r \mid a_{u}\right\rangle}+1\right)} .
\end{aligned}
$$

These identities are justified at the end of this section. Using these and the definition (3.38), we can simplify (C.9) to

$$
\begin{aligned}
& c_{(\cdot)}\left[\sum_{i=1}^{K} m_{\Delta_{i}}^{2}-m_{\Delta_{\delta_{K-1}}}^{2}-2 \sum_{1 \leq r<s \leq K}\left(m_{\Delta_{\langle r \mid s\rangle}}^{2}+\Delta_{\langle r \mid s\rangle} \sum_{\ell=1}^{n-2}\left(\Delta_{\left\langle r \mid a_{\ell}\right\rangle}+\Delta_{\left\langle s \mid a_{\ell}\right\rangle}\right)\right.\right. \\
& \left.\left.-\sum_{\ell=1}^{n-2} \Delta_{\left\langle r \mid a_{\ell}\right\rangle} \Delta_{\left\langle s \mid a_{\ell}\right\rangle}-\sum_{K+1 \leq a_{u}<a_{v} \leq n}\left(\Delta_{\left\langle r \mid a_{u}\right\rangle} \Delta_{\left\langle s \mid a_{v}\right\rangle}+\Delta_{\left\langle r \mid a_{v}\right\rangle} \Delta_{\left\langle s \mid a_{u}\right\rangle}\right)\right)\right] \\
& -4 \sum_{1 \leq r<s \leq K} \widetilde{c}_{\left(k_{s-1}, s ; a_{u}, a_{v}\right.} \sum_{\left.k_{s}-1, \ldots, k_{a_{u}-2}-1, j_{\langle r \mid s\rangle}-1, j_{\left\langle a_{u} \mid a_{v}\right\rangle}-1, j_{\left\langle s \mid a_{u}\right\rangle}+1, j_{\left\langle r \mid a_{v}\right\rangle}+1\right)} \stackrel{!}{=} 0 . \\
& K+1 \leq a_{u}<a_{v} \leq n
\end{aligned}
$$

Now, using the explicit expressions for the dimensions, one can further simplify (C.12) to (3.40). This is straightforward to work out for any given value of $n$ and $K$ (see the supplementary material for calculational details).

To close this section, let's return to the as yet unproven symmetry transformations (C.10)-(C.11). Consider for example, the first identity in (C.11). To prove it, it is useful to take a closer look at the coefficients (3.8) and the dimensions $\Delta_{\langle i \mid j\rangle}$ which can be visually read off of (3.7). Consider the left hand side first, where we need to study the effect of shifting the integral parameters $j_{\left\langle r \mid a_{u}\right\rangle} \rightarrow j_{\left\langle r \mid a_{u}\right\rangle}-1, j_{\left\langle s \mid a_{u}\right\rangle} \rightarrow j_{\left\langle s \mid a_{u}\right\rangle}+1$ in the scaled coefficient $\widetilde{c}_{(\cdot)}^{r, s ; a_{u}, n}$, for fixed $r, s, a_{u}$ satisfying $1 \leq r<s \leq K$ and $K+1 \leq a_{u} \leq n-1$. First assume $r \geq 2$ - we will come back to the case $r=1$ at the end:

- The factor of $\left(\Delta_{1 n, 2 \ldots(n-1)}\right)_{-\sum_{2 \leq \ell_{1}<\ell_{2} \leq n-1} j_{\left\langle\ell_{1} \mid \ell_{2}\right\rangle}}$ in the coefficient (3.8) remains unchanged since the sum over all $j$-parameters is invariant under this shift. 
- Under the given shift, among other effects discussed below, the coefficient (3.8) picks up an overall factor of

$$
\frac{j_{\left\langle r \mid a_{u}\right\rangle} !}{\left(j_{\left\langle r \mid a_{u}\right\rangle}-1\right) !} \frac{j_{\left\langle s \mid a_{u}\right\rangle} !}{\left(j_{\left\langle s \mid a_{u}\right\rangle}+1\right) !}=\frac{j_{\left\langle r \mid a_{u}\right\rangle}}{j_{\left\langle s \mid a_{u}\right\rangle}+1}=\frac{-\Delta_{\left\langle r \mid a_{u}\right\rangle}}{j_{\left\langle s \mid a_{u}\right\rangle}+1},
$$

where we used $\Delta_{\left\langle r \mid a_{u}\right\rangle}=-j_{\left\langle r \mid a_{u}\right\rangle}$ for $r \geq 2$. Moreover note that the scaled coefficient (3.38) comes with additional factors of dimensions $\Delta_{\langle r \mid n\rangle} \Delta_{\left\langle s \mid a_{u}\right\rangle}$. We will address the first factor shortly, but the other one can be read off of (3.7) to be,

$$
\Delta_{\left\langle s \mid a_{u}\right\rangle} \mid \begin{aligned}
& j_{\left\langle r \mid a_{u}\right\rangle} \rightarrow j_{\left\langle r \mid a_{u}\right\rangle}-1 \\
& j_{\left\langle s \mid a_{u}\right\rangle} \rightarrow j_{\left\langle s \mid a_{u}\right\rangle}+1
\end{aligned}=-j_{\left\langle s \mid a_{u}\right\rangle}-1,
$$

where we have appropriately shifted it as required by the left hand side being evaluated. This will cancel the factor in the denominator of the previous displayed equation.

- Looking at the structure of the coefficient (3.8), the integer shifts may potentially affect certain factors for the choice of the dummy parameter $t+2=a_{u}$ in (3.8). However, once again the \pm 1 shifts cancel among each other leaving the following factor invariant

$$
\begin{aligned}
\left.\left(\Delta_{a_{u} \delta_{a_{u}-2}, \delta_{a_{u}-1}}\right)_{\left.k_{a_{u}-2, a_{u}-1}+\sum_{\ell=2}^{a_{u}-1} j_{\left\langle\ell \mid a_{u}\right\rangle}\right\rangle}\right|_{\substack{j_{\left\langle r \mid a_{u}\right\rangle} \rightarrow j_{\left\langle r \mid a_{u}\right\rangle}-1 \\
j_{\left\langle s \mid a_{u}\right\rangle} \rightarrow j_{\left\langle s \mid a_{u}\right\rangle}+1}} & \left(\Delta_{a_{u} \delta_{a_{u}-2}, \delta_{a_{u}-1}}\right)_{k_{a_{u}-2, a_{u}-1}+\sum_{\ell=2}^{a_{u}-1} j_{\left\langle\ell \mid a_{u}\right\rangle}} .
\end{aligned}
$$

On the other hand, when $t+2=r$ or $s$, upon integer shifting as prescribed, one picks up extra overall terms. For $t+2=r$, the following factor, which has been rewritten more suggestively, transforms as,

$$
\begin{aligned}
\left.\frac{\Gamma\left(\Delta_{\langle r \mid n\rangle}\right)}{\Gamma\left(\Delta_{\left.r \delta_{r-1}, \delta_{r-2}\right)}\right)}\right|_{\substack{j_{\left\langle r \mid a_{u}\right\rangle} \rightarrow j_{\left\langle r \mid a_{u}\right\rangle}-1 \\
j_{\left\langle s \mid a_{u}\right\rangle} \rightarrow j_{\left\langle s \mid a_{u}\right\rangle}+1}}=\frac{\Gamma\left(\Delta_{r \delta_{r-1}, \delta_{r-2}}+k_{r-1, r-2}+\sum_{\ell=r+1}^{n-1} j_{\langle r \mid \ell\rangle}-1\right)}{\Gamma\left(\Delta_{r \delta_{r-1}, \delta_{r-2}}\right)} \\
=\frac{\Gamma\left(\Delta_{\langle r \mid n\rangle}-1\right)}{\Gamma\left(\Delta_{\left.r \delta_{r-1}, \delta_{r-2}\right)}\right.}=\frac{1}{\Delta_{\langle r \mid n\rangle}-1} \frac{\Gamma\left(\Delta_{\langle r \mid n\rangle}\right)}{\Gamma\left(\Delta_{\left.r \delta_{r-1}, \delta_{r-2}\right)}\right)} .
\end{aligned}
$$

Now recall the extra factor of $\Delta_{\langle r \mid n\rangle}$ mentioned above, which comes packaged in the scaled coefficient. Under the given shift,

$$
\left.\Delta_{\langle r \mid n\rangle}\right|_{\substack{\left\langle r \mid a_{u}\right\rangle \\ j_{\left\langle s \mid a_{u}\right\rangle} \rightarrow j_{\left\langle r \mid a_{u}\right\rangle}-1}}=\Delta_{\left\langle r \mid a_{u}\right\rangle}+1=1,
$$

which precisely cancels the factor in the denominator of (C.14). Likewise for $t+2=s$, the following factor changes as follows:

$$
\begin{aligned}
\left.\frac{\Gamma\left(\Delta_{\langle s \mid n\rangle}\right)}{\Gamma\left(\Delta_{\left.s \delta_{s-1}, \delta_{s-2}\right)}\right)}\right|_{\substack{j_{\left\langle r \mid a_{u}\right\rangle} \rightarrow j_{\left\langle r \mid a_{u}\right\rangle}-1 \\
j_{\left\langle s \mid a_{u}\right\rangle} \rightarrow j_{\left\langle s \mid a_{u}\right\rangle}+1}}=\frac{\Gamma\left(\Delta_{s \delta_{s-1}, \delta_{s-2}}+k_{s-1, s-2}+\sum_{\ell=s+1}^{n-1} j_{\langle s \mid \ell\rangle}+1\right)}{\Gamma\left(\Delta_{s \delta_{s-1}, \delta_{s-2}}\right)} \\
=\frac{\Gamma\left(\Delta_{\langle s \mid n\rangle}+1\right)}{\Gamma\left(\Delta_{\left.s \delta_{s-1}, \delta_{s-2}\right)}\right.}=\Delta_{\langle s \mid n\rangle} \frac{\Gamma\left(\Delta_{\langle s \mid n\rangle}\right)}{\Gamma\left(\Delta_{s \delta_{s-1}, \delta_{s-2}}\right)} .
\end{aligned}
$$


Putting everything together, upon evaluting the left hand side of the first identity of (C.11), we have recovered the original unshifted coefficient (3.8) times an overall factor of $\Delta_{\left\langle r \mid a_{u}\right\rangle} \Delta_{\langle s \mid n\rangle}$. This is precisely the right hand side of the first identity of (C.11).

Returning to the case of $r=1$, the integer parameter $j_{\langle 1 \mid \ell\rangle}$ is undefined, thus one of the shifts becomes vacuous. Consequently, in contrast with the analysis above for $r \geq 2$, the factor of $\left(\Delta_{1 n, 2 \ldots(n-1)}\right)_{-\sum_{2 \leq r<s \leq n-1} j_{\langle r \mid s\rangle}}$ in the coefficient (3.8) is no longer invariant, but transforms as

$$
\left.\left(\Delta_{1 n, 2 \ldots(n-1)}\right)_{-\sum_{2 \leq \ell_{1}<\ell_{2} \leq n-1} j_{\left\langle\ell_{1} \mid \ell_{2}\right\rangle}}\right|_{j_{\left\langle s \mid a_{u}\right\rangle} \rightarrow j_{\left\langle s \mid a_{u}\right\rangle}+1}=\frac{\left(\Delta_{1 n, 2 \ldots(n-1)}\right)_{-\sum_{2 \leq \ell_{1}<\ell_{2} \leq n-1} j_{\left\langle\ell_{1} \mid \ell_{2}\right\rangle}}}{\Delta_{\langle 1 \mid n\rangle}-1} .
$$

The denominator above is cancelled by the factor of

$$
\left.\Delta_{\langle 1 \mid n\rangle}\right|_{j_{\left\langle s \mid a_{u}\right\rangle} \rightarrow j_{\left\langle s \mid a_{u}\right\rangle}+1}=\Delta_{\langle 1 \mid n\rangle}-1
$$

which comes prepackaged with the scaled coefficient on the left hand side of the identity. The argument for the cancellation of the factor of $\Delta_{\left\langle s \mid a_{u}\right\rangle}=-j_{\left\langle s \mid a_{u}\right\rangle}-1$ is unaffected (the overall minus sign gets cancelled due to the factor of $(-1)^{j_{\left\langle s \mid a_{u}\right\rangle}}$ in (3.8)), and so is the argument for the appearance of an overall factor of $\Delta_{\langle s \mid n\rangle}$ since these do not depend on the integer shift rendered vacuous when $r=1$. Finally, the following factor in the coefficient (3.8) for $t+2=a_{u}$, which previously was invariant, instead transforms as

$$
\begin{aligned}
& \left.\left(\Delta_{a_{u} \delta_{a_{u}-2}, \delta_{a_{u}-1}}\right)_{k_{a_{u}-2, a_{u}-1}+\sum_{\ell=2}^{a_{u}-1} j_{\left\langle\ell \mid a_{u}\right\rangle}}\right|_{j_{\left\langle s \mid a_{u}\right\rangle} \rightarrow j_{\left\langle s \mid a_{u}\right\rangle}+1} \\
& \quad=\left(\Delta_{a_{u} \delta_{a_{u}-2}, \delta_{a_{u}-1}}\right)_{k_{a_{u}-2, a_{u}-1}+\sum_{\ell=2}^{a_{u}-1} j_{\left\langle\ell \mid a_{u}\right\rangle}} \Delta_{\left\langle 1 \mid a_{u}\right\rangle} .
\end{aligned}
$$

Combining all these observations, once again, the left hand side evaluates to the unshifted coefficient (3.8) times a factor of $\Delta_{\left\langle 1 \mid a_{u}\right\rangle} \Delta_{\langle s \mid n\rangle}$, which is precisely the expected right hand side for $r=1$.

The other identities in (C.10)-(C.11) can be proven via very similar analyses. In particular, the proofs for the third identity in both (C.10) and (C.11) also go through without further difficulty, since the $k_{i}$ integer-shifts are uniform across both the left and right hand sides.

Open Access. This article is distributed under the terms of the Creative Commons Attribution License (CC-BY 4.0), which permits any use, distribution and reproduction in any medium, provided the original author(s) and source are credited.

\section{References}

[1] J.M. Maldacena, The large $N$ limit of superconformal field theories and supergravity, Int. J. Theor. Phys. 38 (1999) 1113 [hep-th/9711200] [INSPIRE].

[2] S.S. Gubser, I.R. Klebanov and A.M. Polyakov, Gauge theory correlators from noncritical string theory, Phys. Lett. B 428 (1998) 105 [hep-th/9802109] [InSPIRE]. 
[3] E. Witten, Anti-de Sitter space and holography, Adv. Theor. Math. Phys. 2 (1998) 253 [hep-th/9802150] [INSPIRE].

[4] S. Ferrara, A.F. Grillo and R. Gatto, Tensor representations of conformal algebra and conformally covariant operator product expansion, Annals Phys. 76 (1973) 161 [INSPIRE].

[5] A.M. Polyakov, Nonhamiltonian approach to conformal quantum field theory, Zh. Eksp. Teor. Fiz. 66 (1974) 23 [INSPIRE].

[6] R. Rattazzi, V.S. Rychkov, E. Tonni and A. Vichi, Bounding scalar operator dimensions in 4D CFT, JHEP 12 (2008) 031 [arXiv:0807.0004] [INSPIRE].

[7] D. Poland, S. Rychkov and A. Vichi, The conformal bootstrap: theory, numerical techniques and applications, Rev. Mod. Phys. 91 (2019) 015002 [arXiv: 1805.04405] [INSPIRE].

[8] S. Ferrara, A.F. Grillo and R. Gatto, Manifestly conformal covariant operator-product expansion, Lett. Nuovo Cim. 2S2 (1971) 1363 [INSPIRE].

[9] S. Ferrara, A.F. Grillo, G. Parisi and R. Gatto, Covariant expansion of the conformal four-point function, Nucl. Phys. B 49 (1972) 77 [Erratum ibid. B 53 (1973) 643] [INSPIRE].

[10] S. Ferrara, R. Gatto and A.F. Grillo, Properties of partial wave amplitudes in conformal invariant field theories, Nuovo Cim. A 26 (1975) 226 [INSPIRE].

[11] F.A. Dolan and H. Osborn, Conformal four point functions and the operator product expansion, Nucl. Phys. B 599 (2001) 459 [hep-th/0011040] [INSPIRE].

[12] F.A. Dolan and H. Osborn, Conformal partial waves and the operator product expansion, Nucl. Phys. B 678 (2004) 491 [hep-th/0309180] [INSPIRE].

[13] F.A. Dolan and H. Osborn, Conformal partial waves: further mathematical results, arXiv:1108.6194 [INSPIRE].

[14] A.B. Zamolodchikov, Conformal symmetry in two-dimensions: an explicit recurrence formula for the conformal partial wave amplitude, Commun. Math. Phys. 96 (1984) 419 [INSPIRE].

[15] F. Kos, D. Poland and D. Simmons-Duffin, Bootstrapping the $O(N)$ vector models, JHEP 06 (2014) 091 [arXiv: 1307.6856] [INSPIRE].

[16] J. Penedones, E. Trevisani and M. Yamazaki, Recursion relations for conformal blocks, JHEP 09 (2016) 070 [arXiv: 1509.00428] [INSPIRE].

[17] L. Iliesiu et al., Fermion-scalar conformal blocks, JHEP 04 (2016) 074 [arXiv:1511.01497] [INSPIRE].

[18] M.S. Costa, T. Hansen, J. Penedones and E. Trevisani, Radial expansion for spinning conformal blocks, JHEP 07 (2016) 057 [arXiv: 1603.05552] [INSPIRE].

[19] M.S. Costa, T. Hansen, J. Penedones and E. Trevisani, Projectors and seed conformal blocks for traceless mixed-symmetry tensors, JHEP 07 (2016) 018 [arXiv: 1603. 05551] [INSPIRE].

[20] P. Kravchuk, Casimir recursion relations for general conformal blocks, JHEP 02 (2018) 011 [arXiv: 1709.05347] [INSPIRE].

[21] R.S. Erramilli, L.V. Iliesiu and P. Kravchuk, Recursion relation for general 3d blocks, JHEP 12 (2019) 116 [arXiv:1907.11247] [INSPIRE].

[22] D. Simmons-Duffin, Projectors, shadows and conformal blocks, JHEP 04 (2014) 146 [arXiv: 1204.3894] [INSPIRE]. 
[23] M.S. Costa, J. Penedones, D. Poland and S. Rychkov, Spinning conformal correlators, JHEP 11 (2011) 071 [arXiv: 1107.3554] [InSPIRE].

[24] M.S. Costa, J. Penedones, D. Poland and S. Rychkov, Spinning conformal blocks, JHEP 11 (2011) 154 [arXiv:1109.6321] [INSPIRE].

[25] A. Castedo Echeverri, E. Elkhidir, D. Karateev and M. Serone, Deconstructing conformal blocks in 4D CFT, JHEP 08 (2015) 101 [arXiv: 1505.03750] [INSPIRE].

[26] A. Castedo Echeverri, E. Elkhidir, D. Karateev and M. Serone, Seed conformal blocks in $4 D$ CFT, JHEP 02 (2016) 183 [arXiv: 1601.05325] [INSPIRE].

[27] D. Karateev, P. Kravchuk and D. Simmons-Duffin, Weight shifting operators and conformal blocks, JHEP 02 (2018) 081 [arXiv:1706.07813] [INSPIRE].

[28] G.F. Cuomo, D. Karateev and P. Kravchuk, General bootstrap equations in $4 D$ CFTs, JHEP 01 (2018) 130 [arXiv:1705.05401] [INSPIRE].

[29] H. Isono, On conformal correlators and blocks with spinors in general dimensions, Phys. Rev. D 96 (2017) 065011 [arXiv: 1706. 02835] [INSPIRE].

[30] J.-F. Fortin and W. Skiba, Conformal bootstrap in embedding space, Phys. Rev. D 93 (2016) 105047 [arXiv: 1602 . 05794] [INSPIRE].

[31] J.-F. Fortin and W. Skiba, A recipe for conformal blocks, arXiv:1905.00036 [INSPIRE].

[32] J.-F. Fortin and W. Skiba, New methods for conformal correlation functions, arXiv: 1905.00434 [INSPIRE].

[33] J.-F. Fortin, V. Prilepina and W. Skiba, Conformal four-point correlation functions from the operator product expansion, arXiv: 1907.10506 [INSPIRE].

[34] C. Sleight and M. Taronna, Spinning Witten diagrams, JHEP 06 (2017) 100 [arXiv: 1702.08619] [INSPIRE].

[35] M.S. Costa and T. Hansen, AdS weight shifting operators, JHEP 09 (2018) 040 [arXiv: 1805.01492] [INSPIRE].

[36] M. Besken, A. Hegde, E. Hijano and P. Kraus, Holographic conformal blocks from interacting Wilson lines, JHEP 08 (2016) 099 [arXiv: 1603.07317] [INSPIRE].

[37] A. Bhatta, P. Raman and N.V. Suryanarayana, Holographic conformal partial waves as gravitational open Wilson networks, JHEP 06 (2016) 119 [arXiv:1602.02962] [INSPIRE].

[38] A. Bhatta, P. Raman and N.V. Suryanarayana, Scalar blocks as gravitational Wilson networks, JHEP 12 (2018) 125 [arXiv: 1806.05475] [INSPIRE].

[39] M. Isachenkov and V. Schomerus, Superintegrability of d-dimensional conformal blocks, Phys. Rev. Lett. 117 (2016) 071602 [arXiv: 1602.01858] [INSPIRE].

[40] V. Schomerus, E. Sobko and M. Isachenkov, Harmony of spinning conformal blocks, JHEP 03 (2017) 085 [arXiv: 1612.02479] [INSPIRE].

[41] I. Buric, V. Schomerus and E. Sobko, Superconformal blocks: general theory, JHEP 01 (2020) 159 [arXiv: 1904.04852] [InSPIRE].

[42] I. Burić, V. Schomerus and M. Isachenkov, Conformal group theory of tensor structures, arXiv: 1910.08099 [INSPIRE].

[43] E. Hijano, P. Kraus, E. Perlmutter and R. Snively, Witten diagrams revisited: the AdS geometry of conformal blocks, JHEP 01 (2016) 146 [arXiv: 1508.00501] [INSPIRE]. 
[44] M. Nishida and K. Tamaoka, Geodesic Witten diagrams with an external spinning field, PTEP 2017 (2017) 053B06 [arXiv: 1609. 04563] [INSPIRE].

[45] A. Castro, E. Llabrés and F. Rejon-Barrera, Geodesic diagrams, gravitational interactions 83 OPE structures, JHEP 06 (2017) 099 [arXiv:1702.06128] [INSPIRE].

[46] E. Dyer, D.Z. Freedman and J. Sully, Spinning geodesic Witten diagrams, JHEP 11 (2017) 060 [arXiv: 1702.06139] [INSPIRE].

[47] H.-Y. Chen, E.-J. Kuo and H. Kyono, Anatomy of geodesic Witten diagrams, JHEP 05 (2017) 070 [arXiv: 1702.08818] [INSPIRE].

[48] S.S. Gubser and S. Parikh, Geodesic bulk diagrams on the Bruhat-Tits tree, Phys. Rev. D 96 (2017) 066024 [arXiv:1704.01149] [INSPIRE].

[49] P. Kraus et al., Witten diagrams for torus conformal blocks, JHEP 09 (2017) 149 [arXiv: 1706.00047] [INSPIRE].

[50] K. Tamaoka, Geodesic Witten diagrams with antisymmetric tensor exchange, Phys. Rev. D 96 (2017) 086007 [arXiv:1707.07934] [INSPIRE].

[51] M. Nishida and K. Tamaoka, Fermions in geodesic Witten diagrams, JHEP 07 (2018) 149 [arXiv: 1805.00217] [INSPIRE].

[52] S. Das, Comments on spinning OPE blocks in AdS $3 / C F T_{2}$, Phys. Lett. B 792 (2019) 397 [arXiv: 1811.09375] [INSPIRE].

[53] V. Rosenhaus, Multipoint conformal blocks in the comb channel, JHEP 02 (2019) 142 [arXiv: 1810.03244] [INSPIRE].

[54] V. Gonçalves, R. Pereira and X. Zhou, $20^{\prime}$ five-point function from $A d S_{5} \times S^{5}$ supergravity, JHEP 10 (2019) 247 [arXiv: 1906.05305] [INSPIRE].

[55] S. Parikh, Holographic dual of the five-point conformal block, JHEP 05 (2019) 051 [arXiv: 1901.01267] [INSPIRE].

[56] C.B. Jepsen and S. Parikh, Propagator identities, holographic conformal blocks and higher-point AdS diagrams, JHEP 10 (2019) 268 [arXiv:1906.08405] [INSPIRE].

[57] Z. Komargodski and A. Zhiboedov, Convexity and liberation at large spin, JHEP 11 (2013) 140 [arXiv: 1212.4103] [INSPIRE].

[58] A.L. Fitzpatrick, J. Kaplan, D. Poland and D. Simmons-Duffin, The analytic bootstrap and AdS superhorizon locality, JHEP 12 (2013) 004 [arXiv:1212.3616] [INSPIRE].

[59] A.L. Fitzpatrick, J. Kaplan and M.T. Walters, Universality of long-distance AdS physics from the CFT bootstrap, JHEP 08 (2014) 145 [arXiv: 1403.6829] [INSPIRE].

[60] A. Kaviraj, K. Sen and A. Sinha, Analytic bootstrap at large spin, JHEP 11 (2015) 083 [arXiv: 1502.01437] [INSPIRE].

[61] A. Kaviraj, K. Sen and A. Sinha, Universal anomalous dimensions at large spin and large twist, JHEP 07 (2015) 026 [arXiv:1504.00772] [INSPIRE].

[62] L.F. Alday and A. Zhiboedov, An algebraic approach to the analytic bootstrap, JHEP 04 (2017) 157 [arXiv: 1510.08091] [INSPIRE].

[63] L.F. Alday, Large spin perturbation theory for conformal field theories, Phys. Rev. Lett. 119 (2017) 111601 [arXiv:1611.01500] [INSPIRE]. 
[64] D. Simmons-Duffin, The lightcone bootstrap and the spectrum of the 3d Ising CFT, JHEP 03 (2017) 086 [arXiv : 1612.08471] [INSPIRE].

[65] S. Caron-Huot, Analyticity in spin in conformal theories, JHEP 09 (2017) 078 [arXiv: 1703.00278] [INSPIRE].

[66] S. Albayrak, D. Meltzer and D. Poland, More analytic bootstrap: nonperturbative effects and fermions, JHEP 08 (2019) 040 [arXiv:1904.00032] [INSPIRE].

[67] A. Gadde, Vector space of CFTs, talk given at International Centre for Theoretical Sciences, May 30, Bengaluru, India (2018).

[68] A.L. Fitzpatrick et al., A natural language for AdS/CFT correlators, JHEP 11 (2011) 095 [arXiv:1107.1499] [INSPIRE].

[69] M.F. Paulos, Towards Feynman rules for Mellin amplitudes, JHEP 10 (2011) 074 [arXiv: 1107.1504] [INSPIRE].

[70] D. Nandan, A. Volovich and C. Wen, On Feynman rules for Mellin amplitudes in AdS/CFT, JHEP 05 (2012) 129 [arXiv:1112.0305] [INSPIRE].

[71] S.S. Gubser et al., p-adic AdS/CFT, Commun. Math. Phys. 352 (2017) 1019 [arXiv: 1605.01061] [INSPIRE].

[72] M. Heydeman, M. Marcolli, I. Saberi and B. Stoica, Tensor networks, p-adic fields and algebraic curves: arithmetic and the $A d S_{3} / C F T_{2}$ correspondence, Adv. Theor. Math. Phys. 22 (2018) 93 [arXiv: 1605.07639] [INSPIRE].

[73] F.Q. Gouvêa, p-adic numbers: an introduction, Springer, Germany (1997).

[74] E. Melzer, Nonarchimedean conformal field theories, Int. J. Mod. Phys. A 4 (1989) 4877 [INSPIRE].

[75] S.S. Gubser et al., Edge length dynamics on graphs with applications to p-adic AdS/CFT, JHEP 06 (2017) 157 [arXiv: 1612.09580] [INSPIRE].

[76] S.S. Gubser, C. Jepsen, S. Parikh and B. Trundy, $O(N)$ and $O(N)$ and $O(N)$, JHEP 11 (2017) 107 [arXiv: 1703.04202] [INSPIRE].

[77] S.S. Gubser et al., Melonic theories over diverse number systems, Phys. Rev. D 98 (2018) 126007 [arXiv: 1707.01087] [INSPIRE].

[78] P. Dutta, D. Ghoshal and A. Lala, Enhanced Symmetry of the p-adic Wavelets, Phys. Lett. B 783 (2018) 421 [arXiv: 1804.00958] [INSPIRE].

[79] S. Bhowmick and K. Ray, Holography on local fields via Radon Transform, JHEP 09 (2018) 126 [arXiv: 1805.07189] [INSPIRE].

[80] S.S. Gubser, C. Jepsen, Z. Ji and B. Trundy, Continuum limits of sparse coupling patterns, Phys. Rev. D 98 (2018) 045009 [arXiv: 1805.07637] [INSPIRE].

[81] S.S. Gubser, C. Jepsen and B. Trundy, Spin in p-adic AdS/CFT, J. Phys. A 52 (2019) 144004 [arXiv: 1811.02538] [INSPIRE].

[82] S.S. Gubser, C. Jepsen, Z. Ji and B. Trundy, Mixed field theory, JHEP 12 (2019) 136 [arXiv: 1811.12380] [INSPIRE].

[83] C.B. Jepsen and S. Parikh, p-adic Mellin amplitudes, JHEP 04 (2019) 101 [arXiv: 1808.08333] [INSPIRE]. 
[84] C.B. Jepsen and S. Parikh, Recursion relations in p-adic Mellin space, J. Phys. A 52 (2019) 285401 [arXiv: 1812.09801] [INSPIRE].

[85] F. Qu and Y.-h. Gao, Scalar fields on p-AdS, Phys. Lett. B 786 (2018) 165 [arXiv: 1806.07035] [INSPIRE].

[86] M. Heydeman, M. Marcolli, S. Parikh and I. Saberi, Nonarchimedean holographic entropy from networks of perfect tensors, arXiv:1812.04057 [INSPIRE].

[87] L.-Y. Hung, W. Li and C.M. Melby-Thompson, Wilson line networks in p-adic AdS/CFT, JHEP 05 (2019) 118 [arXiv: 1812.06059] [INSPIRE].

[88] L.-Y. Hung, W. Li and C.M. Melby-Thompson, p-adic CFT is a holographic tensor network, JHEP 04 (2019) 170 [arXiv: 1902.01411] [INSPIRE].

[89] A. Huang, B. Stoica and S.-T. Yau, General relativity from p-adic strings, arXiv: 1901.02013 [INSPIRE].

[90] G. Bentsen et al., Treelike interactions and fast scrambling with cold atoms, Phys. Rev. Lett. 123 (2019) 130601 [arXiv:1905.11430] [INSPIRE].

[91] S.S. Gubser et al., Non-local non-linear $\sigma$-models, JHEP 09 (2019) 005 [arXiv:1906.10281] [INSPIRE].

[92] S. Ebert, H.-Y. Sun and M.-Y. Zhang, Probing holography in p-adic CFT, arXiv: 1911.06313 [INSPIRE].

[93] J. Penedones, Writing CFT correlation functions as AdS scattering amplitudes, JHEP 03 (2011) 025 [arXiv: 1011.1485] [INSPIRE].

[94] Functional identities for ${ }_{3} F_{2}$ functions at $z=1$, http://functions.wolfram.com/07.27.17.0041.01. 